\title{
Analytical fusion of multimodal magnetic resonance imaging to identify pathological states in genetically selected Marchigian Sardinian alcohol-preferring (msP) rats
}

\section{Alejandro Cosa Liñán}

\author{
Dissertation submitted \\ in partial fulfillment of the requirements \\ for the degree of

\section{Doctor in Philosophy}

\author{
Supervisors: \\ Dr. David Moratal Pérez \\ Center of Biomaterials and Tissue Engineering \\ Universitat Politècnica de València, Valencia, Spain \\ Dr. Santiago Canals Gamoneda \\ Instituto de Neurociencias \\ Consejo Superior de Investigaciones Científicas - Universidad Miguel Hernández
}

July, 2017
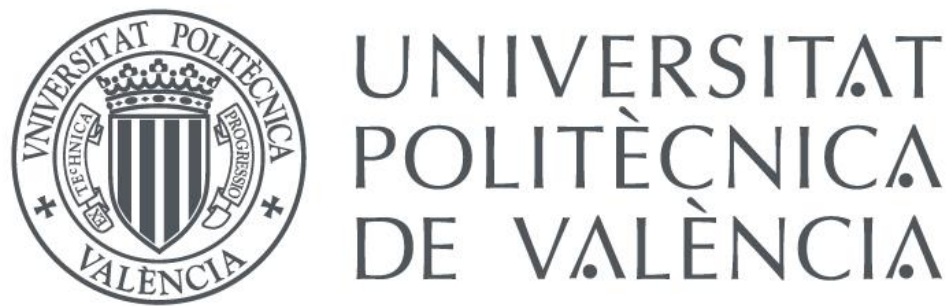

The research described in this thesis was carried out between June 2011 and April 2014 at the Instituto de Neurociencias, Consejo Superior de Investigaciones Científicas \& Universidad Miguel Hernández (Alicante, Spain) and the Centro de Biomateriales and Ingenieria Tisular of the Universitat Politècnica de València. This research project was partially funded by grants from the Spanish MINECO to S.C. (BFU2012-39958, BFU2015-64380-C2-1-R and PIM2010ERN-00679 as part of the Era-Net NEURON TRANSALC) and to D.M. BFU2015-64380-C2-2-R. 



\section{Acknowledgements}

I would like to express my special appreciation and thanks to my advisors Dr. David Moratal and Dr. Santiago Canals for their support and advices along these years. Your encouragement and advices have shaped my research from different scientific perspective making me the research scientist that I am today. Without you this PhD would not have been achievable.

I want also to appreciate to Dr. Wolfgang Sommer for his offer to become part of the Zentral Institute für Seeschliche Gesundheit in Mannheim to progress with my career during my $\mathrm{PhD}$ and afterwards. I also want to extend the warm thanks to Dr. Wolfgang Weber-Fahr for his support during my stay in Mannheim first as PhD student and then, as a researcher. No less appreciated has been the support from Prof. Dr. Hamid R Noori and Prof. Dr. Rainer Spanagel and the entire Psychopharmacology Group and Translational Neuroimaging in Mannheim.

I also want to thank all the members of the TransAlc project and Sybil-AA project for their contributions. Also members Andrea Moreno and Jesus Pacheco of Instituto de Neurociencias-CSIC in Alicante for their experimental and acquisition work and Vicente Pallares for his help. I also acknowledge Dr. Roberto Cicoccioppo for sharing the alcohol preferring msP rats.

I do not forget the people from Centro de Biomateriales and Ingenieria Tisular in the Universitat Politècnica de València for their support during the years I spent with them. Special grateful for Úrsula, Silvia, Irene, Alex, Antonio ... and all of you who shared that time with me.

Por último, quiero agradecer a mis padres, abuelos, tíos, primos y amigos su apoyo incondicional a lo largo de los años. A Dani y Pilar, por nada en concreto, solo por estar cuando os he necesitado.

A ti, Fanny, por hacer todo los caminos en la vida juntos. Por entenderme o por hacer por entenderme, por acompañarme en mis aventuras y desventuras, por dejarlo todo. 



\section{Contents}

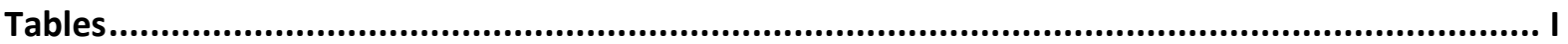

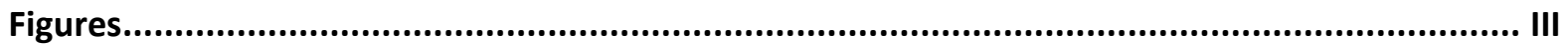

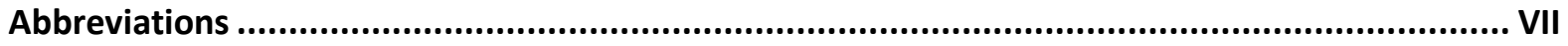

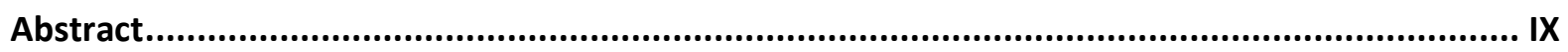

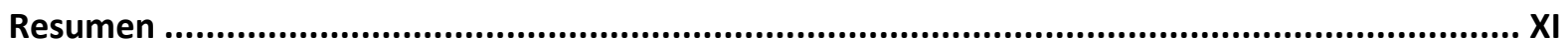

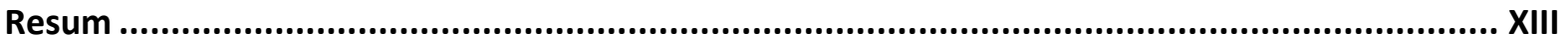

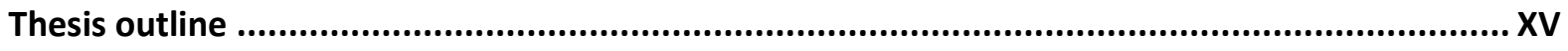

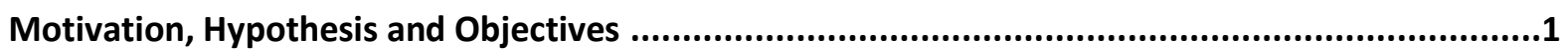

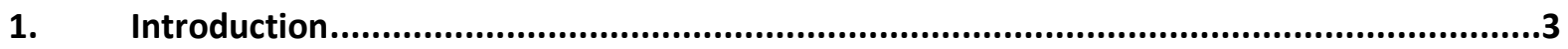

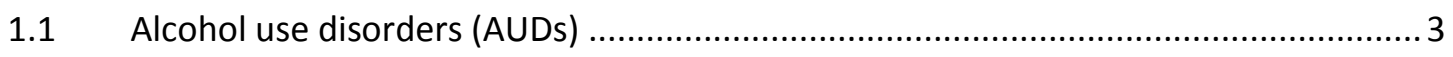

1.1.1 Animal models in alcoholism ............................................................... 7

1.2 Brain imaging to identify neuroanatomical basis of alcohol addiction ........................ 8

1.3 Machine Learning in Brain Imaging .................................................................... 11

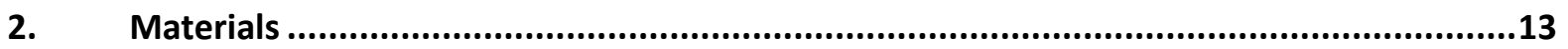

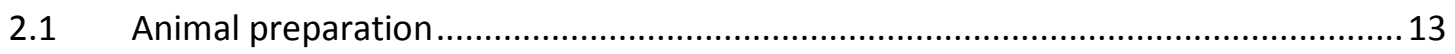

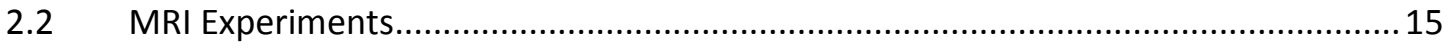

2.3 Software and Computational Tools ….................................................................. 17

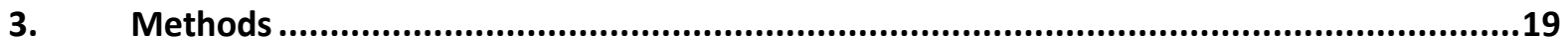

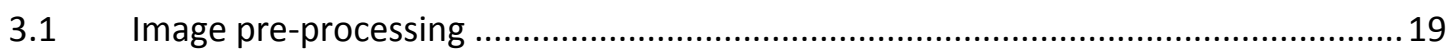

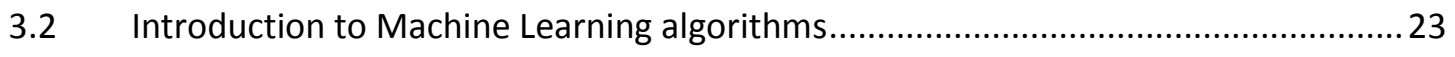

3.2.1 Support Vector Machine classification .........................................................2 23

3.2.2 Feature Extraction and Selection with Random Forest Algorithm ..................27

3.2.3 Validation and possible bias of the predictions ............................................30 
3.3 Multimodal magnetic resonance imaging as a tool for identifying the different stages

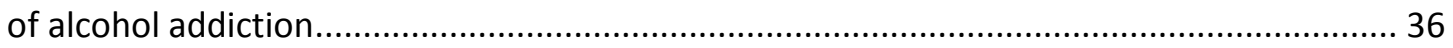

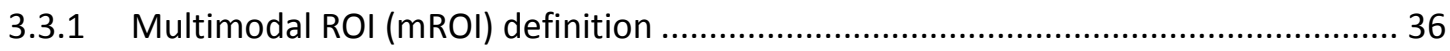

3.3.2 Feature extraction, ranking and support vector machine learning classification...... 40

3.4 A mesoscopic multimodal machine learning approach from alcohol initiation to treatment in msP rats

3.4.1 Feature extraction, ranking and support vector machine learning classification

4. Results.

4.1 Preliminary results

4.2 Multimodal magnetic resonance imaging as a tool for identifying the different stages of alcohol addiction.

4.2.1 Definition of the Finite Mixture Model ......................................................... 48

4.2.2 Multivariate analysis of MSDs .................................................................... 51

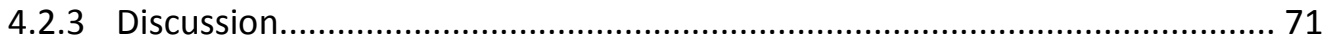

4.3 A mesoscopic multimodal machine learning approach from alcohol initiation to

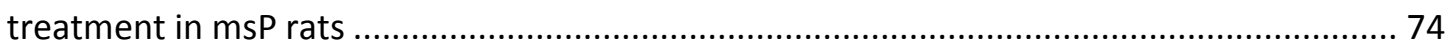

4.3.1 Effect of alcohol consumption in MRI parametric maps ............................... 74

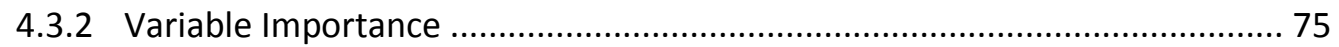

4.3.3 Naïve vs Ethanol Exposed SVM Classification .............................................. 80

4.3.4 Evaluation of treatment efficacy by SVM ................................................ 85

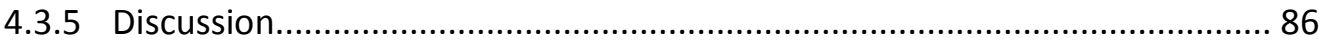

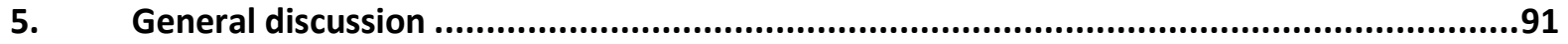

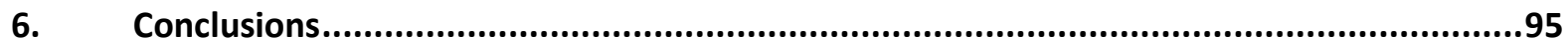

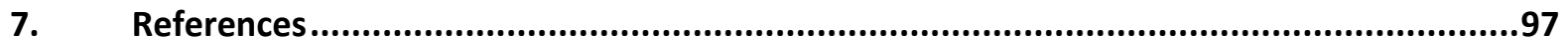

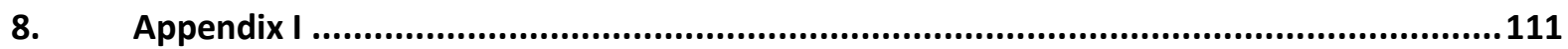




\section{Tables}

Table 4.1 Performance of unimodal and multimodal SVM models to identify msP rats from the Wistar control.

Table 4.2 Performance of unimodal and multimodal SVM models to identify msP rats after one month of heavy alcohol consumption.

Table 4.3 Performance of unimodal and multimodal SVM models to to distinguish between animals in a one month continuous drinking paradigm from the same subjects after a one week of forced abstinence...

Table 4.4 Performance of unimodal and multimodal SVM models to distinguish between animals in a one month continuous drinking paradigm from the same subjects after a one week of naltrexone treatment.

Table 4.5 Performance of unimodal and multimodal SVM models to distinguish abstinent subjects according to Naltrexone or saline treatment. 69

Table 4.6 Variable importance of the MRI maps. .78 


\section{Figures}

Figure 1.1 Alcohol consumption per capita in liters of pure ethanol in Europe ................................. 3

Figure 1.2 Effects of High-Risk Drinking. ................................................................................... 4

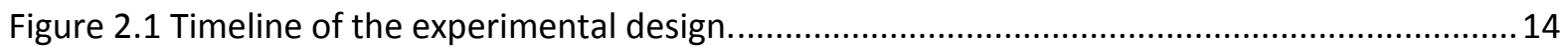

Figure 2.2 Ethanol consumption during one month of free-access................................................ 15

Figure 2.3 Magnetic Resonance scanner at Instituto de Neurociencias in Alicante in San Juan de

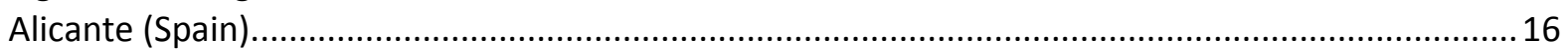

Figure 3.1 Template and tissue probability maps derived from MRI............................................... 22

Figure 3.2 SVM classification. A hyperplane is used to discriminate both classes $[-1,1] \ldots \ldots \ldots \ldots \ldots \ldots . . .25$

Figure 3.3 Hypothetical decision tree to identify classes ............................................................29

Figure 3.4 Classification schemes implemented …..................................................................... 33

Figure 3.5 Schematic representation of probability distribution of mixture of 2 Gaussian components

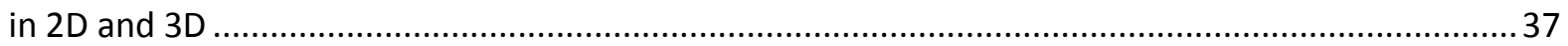

Figure 3.6 Framework of the proposed procedure.................................................................... 42

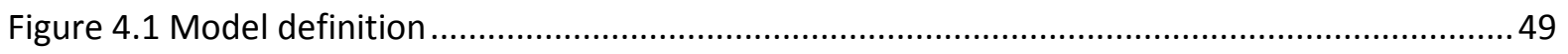

Figure 4.2 Fitting of the 4D data by mixture of seven Student's t distribution..................................50

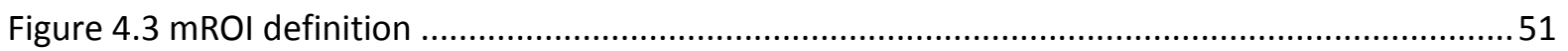

Figure 4.4 PCA decomposition and contribution of MRI-derived features to differentiate

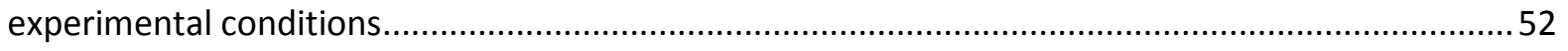

Figure 4.5 Results of the implemented model to distinguish between msP and Wistar subjects.......54

Figure 4.6 Performance of unimodal and multimodal SVM models to identify msP rats from the Wistar control.

Figure 4.7 Feature selection to classify msP rats after one month of heavy alcohol consumption ....57

Figure 4.8 Performance of unimodal and multimodal SVM models to identify msP rats after one month of heavy alcohol consumption. 
Figure 4.9 Feature selection of the implemented model to distinguish between animals in a one month continuous drinking paradigm from the same subjects after a one week of forced abstinence

60

Figure 4.10 Performance of unimodal and multimodal SVM models to distinguish between animals in a one month continuous drinking paradigm from the same subjects after a one week of forced abstinence.

Figure 4.11 Results of the implemented model to distinguish between animals in a one month continuous drinking paradigm from the same subjects after a one week of naltrexone treatment.. 63

Figure 4.12 Performance of unimodal and multimodal SVM models to distinguish between animals in a one month continuous drinking paradigm from the same subjects after a one week of naltrexone treatment. 64

Figure 4.13 Feature selection to classify abstinent subjects according to Naltrexone or saline treatment. 66

Figure 4.14 Performance of unimodal and multimodal SVM models to classify abstinent subjects according to Naltrexone or saline treatment 68

Figure 4.15 Relationship between variable importance and label-permutation tests. Variable importances for discriminating Wistar vs msP rats 70

Figure 4.16 Effect of inclusion of RF feature selection in the inner loop of the cross-validation scheme

Figure 4.17 Effect of alcohol consumption in $\mathrm{msP}$ rats compared of one month of isolation in the home-cage for controlling age effects $(n=17-18)$ 75

Figure 4.18 Importance of IMSD in discriminating Naïve and Exposed experimental states 77

Figure 4.19 Spatial representation of tissue parameter differences between naïve and post-alcohol exposure state.

Figure 4.20 Effect of overfitting in voxelwise classification. 80

Figure 4.21 Comparison between unimodal and multimodal performance.

Figure 4.22 Innate evidences of alcohol preference identified by multimodal neuroimaging ......

Figure 4.23 Probability of being classified as exposed animals strongly depends on initial alcohol preference but not on later preference. 84

Figure 4.24 Effect of naltrexone treatment and saline on the reduction of the prediction of the brain tissue through local multimodal fingerprints. 85 
Figure 4.25 Naltrexone efficacy is correlated to initial alcohol preference in the ventral thalamus but not to steady preference. 


\section{Abbreviations}

\begin{tabular}{|c|c|}
\hline $3 D$ & Tridimensional \\
\hline Acc & Accuracy \\
\hline AIC & Akaike Information Criterion \\
\hline ANOVA & Analysis of variance \\
\hline AUC-ROC & Area under the curve of the receiving-operating characteristic \\
\hline BET & Brain Extraction Tool \\
\hline $\mathrm{BIC}$ & Bayesian Information Criterion \\
\hline BSMCA & Best single modality classifier \\
\hline CSF & Cerebro-spinal fluid \\
\hline CV & Cross-validation \\
\hline DARTEL & Diffeomorphic Anatomical Registration through Exponentiated Algebra \\
\hline DTI & Diffusion Tensor Imaging \\
\hline EPI & Echo Planar Imaging \\
\hline $\mathrm{EtOH}$ & Ethanol \\
\hline FA & Fractional Anisotropy \\
\hline FD & Frame Displacement \\
\hline FOV & Field of view \\
\hline FS & Feature Selection \\
\hline GLM & General Linear Model \\
\hline GM & Gray matter \\
\hline GMv & Gray matter volume \\
\hline GRF & Gaussian random field \\
\hline MD & Mean Diffusivity \\
\hline MSD & Multimodal Signature Disease \\
\hline ML & Machine learning \\
\hline MRI & Magnetic Resonance Imaging \\
\hline $\mathrm{mROI}$ & Multimodal Region of Interest \\
\hline MSMCA & Most important single modality classifier \\
\hline $\mathrm{msP}$ & Marchigian-Sardinian rats \\
\hline RARE & Rapid Acquisition with Relaxation Enhancement \\
\hline RF & Random Forest \\
\hline Sens & Sensitivity \\
\hline SL & Statistical learning \\
\hline Spec & Specificity \\
\hline SPM & Statistical Parametric Map \\
\hline SVM & Support Vector Machine \\
\hline $\mathrm{T} 1$ & Longitudinal relaxation time \\
\hline $\mathrm{T} 2$ & Transversal relaxation time \\
\hline TE & Echo time \\
\hline
\end{tabular}


TPM Tissue probability map

TR Repetition time

VI Variable Importance

WM White matter

WMv White matter volume 


\section{Abstract}

Alcohol abuse is one of the most alarming issues for the health authorities. It is estimated that at least 23 million of European citizens are affected by alcoholism causing a cost around 270 million euros. Excessive alcohol consumption is related with physical harm and, although it damages the most of body organs, liver, pancreas, and brain are more severally affected. Not only physical harm is associated to alcohol-related disorders, but also other psychiatric disorders such as depression are often comorbiding. As well, alcohol is present in many of violent behaviors and traffic injures. Altogether reflects the high complexity of alcohol-related disorders suggesting the involvement of multiple brain systems.

With the emergence of non-invasive diagnosis techniques such as neuroimaging or EEG, many neurobiological factors have been evidenced to be fundamental in the acquisition and maintenance of addictive behaviors, relapsing risk, and validity of available treatment alternatives. Alterations in brain structure and function reflected in non-invasive imaging studies have been repeatedly investigated. However, the extent to which imaging measures may precisely characterize and differentiate pathological stages of the disease often accompanied by other pathologies is not clear. The use of animal models has elucidated the role of neurobiological mechanisms paralleling alcohol misuses. Thus, combining animal research with non-invasive neuroimaging studies is a key tool in the advance of the disorder understanding.

As the volume of data from very diverse nature available in clinical and research settings increases, an integration of data sets and methodologies is required to explore multidimensional aspects of psychiatric disorders. Complementing conventional mass-variate statistics, interests in predictive power of statistical machine learning to neuroimaging data is currently growing among scientific community.

This doctoral thesis has covered most of the aspects mentioned above. Starting from a wellestablished animal model in alcohol research, Marchigian Sardinian rats, we have performed multimodal neuroimaging studies at several stages of alcohol-experimental design including the etiological mechanisms modulating high alcohol consumption (in comparison to Wistar control rats), alcohol consumption, and treatment with the opioid antagonist Naltrexone, a well-established drug 
in clinics but with heterogeneous response. Multimodal magnetic resonance imaging acquisition included Diffusion Tensor Imaging, structural imaging, and the calculation of magnetic-derived relaxometry maps. We have designed an analytical framework based on widely used algorithms in neuroimaging field, Random Forest and Support Vector Machine, combined in a wrapping fashion. Designed approach was applied on the same dataset with two different aims: exploring the validity of the approach to discriminate experimental stages running at subject-level and establishing predictive models at voxel-level to identify key anatomical regions modified during the experiment course.

As expected, combination of multiple magnetic resonance imaging modalities resulted in an enhanced predictive power (between 3 and 16\%) with heterogeneous modality contribution. Surprisingly, we have identified some inborn alterations correlating high alcohol preference and thalamic neuroadaptations related to Naltrexone efficacy. As well, reproducible contribution of DTI and relaxometry -related biomarkers has been repeatedly identified guiding further studies in alcohol research.

In summary, along this research we demonstrate the feasibility of incorporating multimodal neuroimaging, machine learning algorithms, and animal research in the advance of the understanding alcohol-related disorders. 


\section{Resumen}

El abuso de alcohol es una de las mayores preocupaciones de las autoridades sanitarias en la Unión Europea. Aunque resulta difícil de establecer cifras con exactitud, se estima que unos 23 millones de europeos desarrollan patologías derivadas del abuso de alcohol con un coste de unos 150.000 millones de euros para la sociedad. El consumo de alcohol en exceso afecta en mayor o menor medida la totalidad del organismo siendo el páncreas e hígado los más severamente afectados. Además de estos, el sistema nervioso central sufre deterioros relacionados con el alcohol y con frecuencia se presenta en paralelo con otras patologías psiquiátricas como la depresión u otras adicciones como la ludopatía. La presencia de estas comorbidades demuestra la complejidad de la patología en la que multitud de sistemas neuronales interaccionan entre sí.

El uso de herramientas como electroencefalogramas (EEG) o imágenes de resonancia magnética (RM) han ayudado en el estudio de enfermedades psiquiátricas facilitando el descubrimiento de mecanismos neurológicos fundamentales en el desarrollo y mantenimiento de la adicción al alcohol, recaídas y el efecto de los tratamientos disponibles. A pesar de los avances, todavía se necesita investigar más para identificar las bases biológicas que contribuyen a la enfermedad. En este sentido, los modelos animales sirven, por lo tanto, a discriminar aquellos factores únicamente relacionados con el alcohol controlando otros factores que facilitan el desarrollo del alcoholismo. Estudios de resonancia magnética en animales de laboratorio y su posterior evaluación en humanos juegan un papel fundamental en el entendimiento de las patologías psiquatricas como la addicción al alcohol.

La imagen por resonancia magnética se ha integrado en entornos clínicos como prueba diagnósticas no invasivas. A medida que el volumen de datos se va incrementando, se necesitan herramientas y metodologías capaces de fusionar información de muy distinta naturaleza y así establecer criterios diagnósticos cada vez más exactos. El poder predictivo de herramientas derivadas de la inteligencia artificial como el aprendizaje automático sirven de complemento a tradicionales métodos estadísticos.

A lo largo de esta investigación se han abordado la mayoría de estos aspectos. Se han obtenido datos multimodales de resonancia magnética de un modelo validado en la investigación de 
patologías derivadas del consumo del alcohol, las ratas Marchigian-Sardinian desarrolladas en la Universidad de Camerino (Italia) y con consumos de alcohol comparables a los humanos. Para cada animal se han adquirido datos antes y después del consumo de alcohol y bajo dos condiciones de abstinencia (con y sin tratamiento de Naltrexona, una medicaciones anti-recaídas usada como farmacoterapia en el alcoholismo). Los datos de resonancia magnética multimodal consistentes en imágenes de difusión, de relaxometría y estructurales se han fusionado en un esquema analítico multivariable incorporando dos herramientas generalmente usadas en datos derivados de neuroimagen, Random Forest y Support Vector Machine. Nuestro esquema fue aplicado con dos objetivos diferenciados. Por un lado, determinar en qué fase experimental se encuentra el sujeto a partir de biomarcadores y por el otro, identificar sistemas cerebrales susceptibles de alterarse debido a una importante ingesta de alcohol y su evolución durante la abstinencia.

Nuestros resultados demostraron que cuando biomarcadores derivados de múltiples modalidades de neuroimagen se fusionan en un único análisis producen diagnósticos más exactos que los derivados de una única modalidad (hasta un 16\% de mejora). Biomarcadores derivados de imágenes de difusión y relaxometría discriminan estados experimentales. También se han identificado algunos aspectos innatos que están relacionados con posteriores comportamientos con el consumo de alcohol o la relación entre la respuesta al tratamiento y los datos de resonancia magnética.

Resumiendo, a lo largo de esta tesis, se demuestra que el uso de datos de resonancia magnética multimodales en modelos animales combinados en esquemas analíticos multivariados es una herramienta válida en el entendimiento de patologías psiquiátricas como el alcoholismo. 


\section{Resum}

L'abús de alcohol es una de les majors preocupacions per part de les autoritats sanitàries de la Unió Europea. Malgrat la dificultat de establir xifres exactes, se estima que uns 23 milions de europeus actualment sofreixen de malalties derivades del alcoholisme amb un cost que supera els 150.000 milions de euros per a la societat. Un consum de alcohol en excés afecta en major o menor mesura el cos humà sent el pàncreas i el fetge el més afectats. A més, el cervell sofreix de deterioraments produïts per l'alcohol i amb freqüència coexisteixen amb altres patologies com depressió o altres addiccions com la ludopatia. Tot aquest demostra la complexitat de la malaltia en la que múltiple sistemes neuronals interactuen entre si.

Tècniques no invasives com el encefalograma (EEG) o imatges de ressonància magnètica (RM) han ajudat en l'estudi de malalties psiquiàtriques facilitant el descobriment de mecanismes neurològics fonamentals en el desenvolupament i manteniment de la addició, recaiguda i la efectivitat dels tractaments disponibles. Tot i els avanços, encara es necessiten més investigacions per identificar les bases biològiques que contribueixen a la malaltia. En aquesta direcció, el models animals serveixen per a identificar únicament dependents del abús del alcohol. Estudis de ressonància magnètica en animals de laboratori i posterior avaluació en humans jugarien un paper fonamental en l' enteniment de l'ús del alcohol.

L'ús de probes diagnostiques no invasives en entorns clínics has sigut integrades. A mesura que el volum de dades es incrementa, eines i metodologies per a la fusió d' informació de molt distinta natura i per tant, establir criteris diagnòstics cada vegada més exactes. La predictibilitat de eines desenvolupades en el camp de la intel-ligència artificial com la aprenentatge automàtic serveixen de complement a mètodes estadístics tradicionals.

En aquesta investigació se han abordat tots aquestes aspectes. Dades multimodals de ressonància magnètica se han obtingut de un model animal validat en l'estudi de patologies relacionades amb el consum d'alcohol, les rates Marchigian-Sardinian desenvolupades en la Universitat de Camerino (Italià) i amb consums d'alcohol comparables als humans. Per a cada animal es van adquirir dades previs i després al consum de alcohol i dos condicions diferents de abstinència (amb i sense tractament anti-recaiguda). Dades de ressonància magnètica multimodal constituides 
per imatges de difusió, de relaxometria magnètica i estructurals van ser fusionades en esquemes analítics multivariats incorporant dues metodologies validades en el camp de neuroimatge, Random Forest i Support Vector Machine. Nostre esquema ha sigut aplicat amb dos objectius diferenciats. El primer objectiu es determinar en quina fase experimental es troba el subjecte a partir de biomarcadors obtinguts per neuroimatge. Per l'altra banda, el segon objectiu es identificar el sistemes cerebrals susceptibles de ser alterats durant una important ingesta de alcohol i la seua evolució durant la fase del tractament.

El nostres resultats demostraren que l'ús de biomarcadors derivats de varies modalitats de neuroimatge fusionades en un anàlisis multivariat produeixen diagnòstics més exactes que els derivats de una única modalitat (fins un $16 \%$ de millora). Biomarcadors derivats de imatges de difusió i relaxometria van contribuir de distints estats experimentals. També s'han identificat aspectes innats que estan relacionades amb posterior preferències d'alcohol o la relació entre la resposta al tractament anti-recaiguda i les dades de ressonància magnètica.

En resum, al llarg de aquest treball, es demostra que l'ús de dades de ressonància magnètica multimodal en models animals combinats en esquemes analítics multivariats són una eina molt valida en l'enteniment i avanç de patologies psiquiàtriques com l'alcoholisme. 


\section{Thesis outline}

The $\mathrm{PhD}$ thesis report is structured along seven main sections which try to sum up in a concise and organized way to make the text easier to read and interpret. The contents of each section in this PhD Thesis report are structured as follows:

The first section is devoted to Motivation, Hypothesis, and Objectives of the research work.

The Introduction chapter (Chapter 1 ) offers a background of the main concepts appearing along this dissertation. First, the main ideas of the alcohol use disorders and its preclinical research are introduced (Section 1.1). Previous findings in the field of the alcohol research by the use of magnetic resonance imaging are summarized in the Section 1.2. A brief introduction of machine learning and neuroimaging in psychiatric disorders is also provided (Section 1.3).

Material chapter (Chapter 2) includes a description of the animal experimentation and preparation (Section 2.1), MRI acquisitions (Section 2.2), and the software used in the analysis of the data (Section 2.3).

Methods chapter (Chapter 3 ) describes the thesis methodology. Image preprocessing previous to analysis is detailed in Section 3.1. General machine learning algorithms are introduced in section 3.2. Implementation of an analytical framework to cope with multimodal brain imaging is described in section 3.3. The framework is evaluated in order to identify different experimental conditions in two different strategies (Sections 3.4 and 3.5).

Chapter 4 (Results and Discussion) is devoted to show and discuss the main results obtained in this research. Preliminary results of a pilot study are found in Section 4.1. In order to differentiate the findings and results that have been obtained by the proposed methodologies, the results are divided in two chapters (Section 4.2 and 4.3).

General Discussion (Chapter 5) discusses the main results obtained in this thesis as well as further work that could be performed.

Conclusions (Chapter 6) sum up all the findings obtained in this research. 
As part of the research, we performed a pilot MRI experiment in order to establish MRI sequence and the analytical tools. Both methodological and acquisition approach differed substantially with the final methodology presented in this thesis. Such details are found in Appendix I. 


\section{Motivation, Hypothesis and Objectives}

Alcohol abuse is one of the most alarming issues for the health authorities. It is estimated that at least 23 million of European citizens are affected by alcoholism causing a cost around 270 million euros. Excessive alcohol consumption is related with physical harm and, although it damages the most of body organs, liver, pancreas, and brain are more severally affected.

Alcohol addiction, as other substances of abuse, is characterized by frequent episodes of intoxication, preoccupation with alcohol, intake of alcohol despite the adverse consequences, compulsion to seek (craving) and consume alcohol, loss of control in limiting the amount of alcohol intake and the emergence of negatives emotional states when the access to the drug is not available. These aspects of the pathology can affect alcoholics in their emotional stability, finances, career, and the ability to build and have good relationships with the others. Despite the efforts, alcoholism treatment options are very limited, with the opioid antagonist naltrexone as a prototypical example providing proof-of-concept for successful pharmacotherapy of alcoholism although with modest effect sizes.

Magnetic resonance imaging (MRI) has provided considerable in vivo evidence of perturbed neural mechanisms underpinning initiation and maintenance of most drugs of abuse. However, despite the substantial advance in the understanding of alcohol misuse by brain imaging techniques, its integration on clinical settings is so far limited, partly because of a lack of reliable factors characterizing pathological states associated to alcohol use and abuse. With the aim to advance the development of pharmacotherapy for alcohol addiction, the project TRANSALC (Translational Neuroimaging in Alcoholism, http://www.transalc.eu) started in March 2011 under a joint funding scheme implemented by ERA-NET NEURON. Specifically, TRANSALC aimed to develop translational in vivo brain imaging tools for improving the predictive value of animal experiments for the development of clinically effective medications in alcohol research.

This dissertation focuses on the assessment at different experimental stages of alcohol abuse in animal models from the inborn aspects which will derive in addiction to structural alterations produced by a chronic abuse. This study takes advantage of all the possibilities that MRI offers by 
univariate and multivariate analysis. The main hypothesis of this work is that the alcoholism can be better understood by the combination of complementary MRI modalities.

To test the main hypothesis, the following objectives are proposed:

1. To develop a methodology that allows discriminating different stages of the alcoholism.

1.1. To merge brain areas according to their multimodal MRI pattern using a probabilistic framework.

1.2. To discriminate different experimental conditions (exposition and treatment) based on multimodal MRI information.

2. To identify brain alterations at different stages of alcoholism using complementary MRI modalities.

2.1. To implement a multivariate voxel-based classification approach in order to identify local changes after an alcohol consumption period.

2.2. To study the recovery of brain alteration caused by alcohol during the withdrawal period. 


\section{Introduction}

\subsection{Alcohol use disorders (AUDs)}

Alcohols, compounds having hydroxyl functional group bound to a carbon atom, have been used as the main ingredient of beverages since early Egyptian civilization. Despite in the 16th Century alcoholic beverages had medicinal purposes, the abuse is one of the most alarming issues in the current European Union. Concretely, the alcohol use is the third leading risk for burden of disease for the health authorities in Europe. Globally, alcohol consumption was behind of about 3.3 million deaths in 2012 representing the $5.9 \%$ of all global deaths. Alcohol addiction is estimated to affect 23 million Europeans ( $5 \%$ of men, $1 \%$ of women), thus probably being the most prevalent neuropsychiatric disorder afflicting our society (1). The World Health Organization emphasizes the high rates of alcohol consumption in the EU where each adult intakes 11 liters of pure alcohol almost doubling the global average. EU citizens engage in harmful drinking with levels of above $20 \mathrm{~g}$ (women) or $40 \mathrm{~g}$ alcohol per day (men) (2).

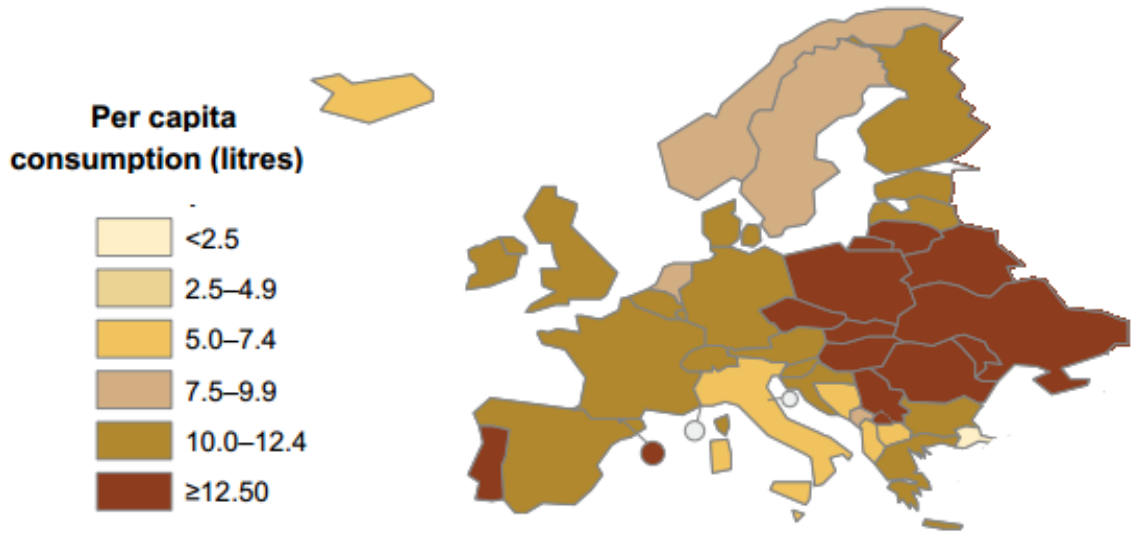

Figure 1.1 Alcohol consumption per capita in liters of pure ethanol in Europe. Adapted from (2)

Alcohol abuse is implicated in a wide variety of diseases, disorders, and injures, as well as many social and legal problems. Physical damage is often resulting of long-term excessive consumption. Although the most of body organs are affected by alcohol intoxication, liver, pancreas, and brain are more severally affected.

The mortality attributable to alcohol consumption is high ( 1 in 7 male deaths and 1 in 13 
female deaths in the adult aged group) despite of the constant awareness campaigns and has been established as one of major risk in the countries of EU (1). The three most important causes of death which are related with the chronic alcohol consumption are cancer (mouth, larynx, pharynx, esophageal, liver, colon, and rectal and female breast cancer), liver cirrhosis, and injuries (3). Moreover, much more common medical complications such as hypertension, gastritis, diabetes and some forms of stroke are aggravated by alcohol abuse, as well as mental disorders such as depression (4). Automobile and pedestrian injures falls and work-related harm result from excessive alcohol consumption. Figure 1.2 summarizes the most common effects of the high alcohol consumption.

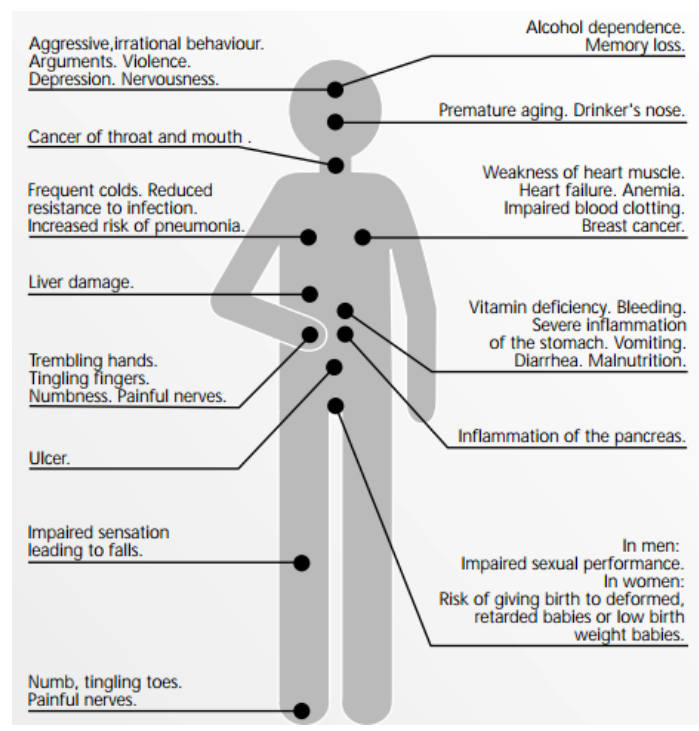

Figure 1.2 Effects of High-Risk Drinking. Reproduced from (5)

Measuring the resultant harms in economic sense is difficult, but a conservative estimate of the annual cost to the EU society for pain, suffering and lost life that occur due to health, social and criminal problems caused by alcohol puts a price to around 150 billion $€$ annually (3). Because its physical damage and economic cost for the society, it is important to understand how alcohol use becomes into misuse. In the following, neurobiological and neuropsychiatric aspects of alcohol misuse will be briefly introduced.

Alcohol-related diseases are not "all or nothing" but it is a chronic, progressive and high-risk of relapsing disorder. At early stages of the disorder, the subject experiments with the alcohol 
experiencing the rewarding aspects of the alcohol consumption (positive reinforcing and euphoria). The positive reinforcing leads to an increase in the frequency and magnitude of drinking. As the disorder advances, the subject presents dysphoric behaviors during periods when alcohol consumption is not available. Dysphoric effects can be physiological (hangover and tachycardia.) or behavioral (negative consequences in career, violence, being arrested during the intoxication). In order to alleviate such negative sensations, dependent subjects often seek alcohol. Therefore, progression of alcohol dependence is characterized by positive reinforcement periods (mainly predominating early stages of experimentation with the drug) and negative reinforcement periods (later stages of the dependency) (6). Thus, from a neuropsychiatric perspective, the alcoholism can be better defined as a pathological behavioral syndrome and its development is characterized by frequent episodes of intoxication, preoccupation with alcohol, intake of alcohol despite the adverse consequences, compulsion to seek (craving) and consume alcohol, loss of control in limiting the amount of alcohol intake and the emergence of negatives emotional states when the access to the drug is not available. Therefore, alcoholics and alcohol abusers can also be affected in their emotional stability, finances, career, and the ability to build and have good relationships with the others. Furthermore, alcohol abuse or dependence can coexists with, contribute to, result from or worse several psychiatric disorders. The mental health problems that most commonly co-occur with substance abuse are depression, anxiety disorders, and bipolar disorder.

Alcohol use disorder is considered a mental disease by the Diagnostic and Statistical Manual of Mental Disorders or DSM (7) and the diagnostic criteria of alcohol use disorder includes:

1. Alcohol is often taken in larger amounts or over a longer period than was intended.

2. There is a persistent desire or unsuccessful efforts to cut down or control alcohol use.

3. A great deal of time is spent in activities necessary to obtain alcohol, use alcohol, or recover from its effects.

4. Craving, or a strong desire or urge to use alcohol.

5. Recurrent alcohol use resulting in a failure to fulfill major role obligations at work, school, or home.

6. Continued alcohol use despite having persistent or recurrent social or interpersonal problems caused or exacerbated by the effects of alcohol. 
7. Important social, occupational, or recreational activities are given up or reduced because of alcohol use.

8. Recurrent alcohol use in situations in which it is physically hazardous.

9. Alcohol use is continued despite knowledge of having a persistent or recurrent physical or psychological problem that is likely to have been caused or exacerbated by alcohol.

10. Tolerance, as defined by either of the following:

a) A need for markedly increased amounts of alcohol to achieve intoxication or desired effect

b) A markedly diminished effect with continued use of the same amount of alcohol.

11. Withdrawal, as manifested by either of the following:

a) The characteristic withdrawal syndrome for alcohol (refer to criteria A and B of the criteria set for alcohol withdrawal)

b) Alcohol (or a closely related substance, such as a benzodiazepine) is taken to relieve or avoid withdrawal symptoms.

These symptoms are often grouped as impaired control over the alcohol (criteria 1-4), social impairment (criteria 5-7), risky use of the substance (criteria 8-9), and pharmacological criteria (criteria 10-11). The presence of at least 2 of these symptoms in the last twelve months is indicative of alcohol use disorder grading mild (2-3 symptoms), moderate (4-5 symptoms), or severe (more than 6 symptoms). Note that diagnosis has changed with respect to previous versions where alcohol abuse and alcohol dependence were considered two distinct entities.

Since only two of the two symptoms are sufficient to diagnose alcohol use disorders, clinical population and alcohol-related effects are highly heterogeneous with different behavioral and physical consequences, where several neurobiological systems (cholinergic, dopaminergic, GABAergic, glutamatergic, serotonergic, noradrenergic, corticotrophin releasing hormone, opioid, and neuropeptide $Y$ ) are thought to mediate independently or interactively modulating acute rewarding and cognitive and behavioral effects and neuroadaptations facilitating addiction $(8,9)$.

Although alcohol addiction is highly prevalent, very few pharmacotherapies are available. Currently disulfiram, naltrexone, acamprosate, nalmefene, and gamma hydroxybutyrate (GHB) are often used for alcohol disorder treatment by prolonging abstinence periods or creating aversive reactions to alcohol. Unfortunately, these medications are not effective in all patients and their 
response is generally modest. The heterogeneity of treatment response is thought to be mediated by patient characteristics and genetic factors (10-12). In this work, we explored the effect of Naltrexone which blocks the release of dopamine into the nucleus accumbens, amygdala, and forebrain. This mechanism reduces the positive reinforcement effects in patients.

Due to the substantial heritable aspect, one of the most important goals of the addiction research on the alcoholism is to understand the neural aspects and the progressive pathological transition between a moderate alcohol use and the alcohol dependence $(13,14)$. Techniques to assess the molecular, cellular, and behavioral events associated with the alcohol dependence in conjunction with animal models that duplicate several aspects of alcohol addiction in humans are widely used in the preclinical research.

\subsubsection{Animal models in alcoholism}

Strictly, an animal model is a preparation in one organism that allows for the study of one or several aspects of a human condition providing insights into the neurobiological, psychological, and/or etiological mechanisms of the human pathology. The most common type of mammal employed in preclinical research is the rodent due to its genome is very similar to that of humans, but also because of the availability, ease to handling, high reproductive rates, and a relative low cost of use and maintenance.

Despite drug abuse in humans is a complex situation in which social and personal reasons as well as behavioral and cognitive deficits prior to drug initiation, animal models provide valuable means to investigate the stages of the addiction including the earlier contact with the drug, the maintenance phase, binges and escalation of drug intake, compulsive drug intake and an inability to inhibit drug-seeking behaviors and continued drug use despite negative or adverse consequences.

It is widely known that rodents consume naturally and voluntary large amounts of alcohol in the laboratory or in the wild setting (usually rotten fruits). This natural behavior positions rats and mice as suitable species to try to understand various aspects of alcohol addiction typical of humans as heavy alcohol consumption. Commonly human alcohol consumption is approached using 
preference studies in rodent, in which animals can choose freely between water and alcohol solutions and the consumed amounts of each fluid are measured (Two-Bottle Free Choice Drinking Paradigm) or other alcohol self-administration paradigms resulting in pharmacologically relevant $\operatorname{BACs}(9,15)$. A subset of this animals present a preference for high alcohol concentrations and this fact allows to researcher to generate pairs of breeds characterized by low or high level alcohol consumption, namely, to classify subjects respect to alcohol preference despite of non-sweet taste. This sort of models represents well-established rodent models, the alcohol-preferring rats. Along this work we based our research in Marchigian Sardinian alcohol-preferring (msP) rats. Starting from the 13th generation of Sardinian alcohol-preferring (sP) rats originally developed at the Department of Neuroscience, University of Camerino, Italy and after 20 generations of selective breeding, the Department of Experimental Medicine and Public Health developed a genetically selected Marchigian Sardinian alcohol-preferring (msP) rat, an established selection-based model for the investigation of the neurobiological aspects of the alcohol addiction (16). In the manner of SP line, $\mathrm{msP}$ rats have a natural preference for ethanol intake but there are differences in genotypic and phenotypic characteristics between them due to the two breeding program were generated under different husbandry conditions and different selection criteria. The Marchigian-Sardinian alcoholpreferring rats mimics several aspects of human disease such as the occurrence of binge-like ethanol drinking, psychological withdrawal symptoms, escalating alcohol intake upon abstinence and highvulnerability to stress-mediated relapse.

The opioid antagonist naltrexone has been investigated in msP rats demonstrating a reduction in EtOH consumption (17). However, new treatments need to surpass the efficacy of naltrexone considered as clinical reference compound on the basis of solid scientific evidence. In the past, discrepancies between pharmacological targets put forward by animal models and the effect of respective drugs in clinical trials have been repeatedly reported (18).

\subsection{Brain imaging to identify neuroanatomical basis of alcohol addiction}

An important aspect of biomedicine system is the identification of biomarkers in medical 
diseases. A biomarker is defined as any biologic feature that can be used to measure or predict the presence or progress of disease or the effects of the treatment $(19,20)$. Over the past three decades, magnetic resonance imaging (MRI) has provided considerable in vivo evidence that structural and functional biomarkers associated with heavy and prolonged exposure to most drugs of abuse. The major findings in addictive behaviors have been done as a result of emergence of neuroimaging techniques in combination with the psychopharmacology and molecular genetics.

Hundreds of studies have employed brain imaging techniques in the advance of the understanding of the neurochemical and neurobiological basis of the addiction and alcoholattributable brain damage. Structural magnetic resonance imaging (sMRI), Diffusion Tensor imaging (DTI), spectroscopy, functional magnetic resonance imaging, and Positron Emission Tomography (PET) are useful to elucidate the neurobiological mechanisms underlying alcohol-related disorders.

Human neuroimaging studies have evidenced regional brain atrophy. Reduced gray matter volume in frontal $(21-23)$ and limbic $(24,25)$ areas has been reduced in alcohol-dependents patients and loss of volume in frontal white matter areas (26). An association between a loss of gray matter volume in reward-related areas and alcoholism has been also reported (25). The extent of volume changes is related to temporal and quantity of alcohol intake patterns $(27,28)$, age $(28)$ and sex of the patient (29-31).

It is well documented that alcohol use disorders are associated with changes in the integrity of white matter tracts. Many previous DTI studies associates the reduction of FA in the genu of the corpus callosum, centrum semiovale (32) and areas in the frontal and superior sites such as frontal forceps, internal and external capsules, and fornix (33) which suggests a demyelination of fiber tract and axonal degeneration (34). Another DTI studies reveal that white matter fibers from midbrain and pons are disrupted by chronic alcohol intake affecting cognitive abilities (35) and abnormalities in motivational and reward related brain areas (36). Alcohol does not affect males and females in the same way. Female brain is more vulnerable to the alcohol effects $(37,38)$. As well as gender, callosal structure is affected by the age of the patient (39).

Kong and colleagues report that changes in the brain after acute alcohol consumption are not permanents and diffusion parameters such as FA and MD are not irreversible(40). The brain edema 
produced for the toxic effect in the brain could be the explanation of these structural changes. Disruptions of brain microstructure seem to be caused by changes in intracellular and extracellular fluid accumulation which restrict water diffusion (34).

MRI relaxometry allows investigating the interaction between molecules and the tissue neurochemistry. For instance, $\mathrm{T} 2$ relaxation time is influenced by the amount of water proton in a specified region $(41,42)$. Prolongation of $\mathrm{T} 2$ relaxation times in central pons has been observed in alcoholics as a contributory mechanism of macrocytic anemia (low red blood count and high mean corpuscular volume) (43). Sheu reports associations between T2 in dopamine targets regions (caudate, putamen, thalamus and dorsolateral prefrontal cortex, nucleus accumbens, substantia nigra) and the use of the drugs and alcohol (44). As well, iron and microglia activation in the brain accumulation (45-47) associated to alcoholism has been established as a cause of reduction (shortening) in T2 relaxation times (48). As well as structural changes, functional reorganization has been probed. Alcohol-dependent subjects differ from healthy controls in brain activation including the cortical areas when the subject is performing several tasks in the scanner.

More recently, a MRI study compares between the Marchigian-Sardinian alcohol preferring rat and wild Wistar rats (genetic control) in order to study the heritable aspects which are responsible of alcohol addiction (49). Decreased gray matter volume in fronto-parietal areas (anterior cingulate and insular cortex), in addition to posterior cingulate (retrosplenial) cortex and the dorso-lateral thalamus and ventral tegmental area were founded. However, no significant differences in the diffusion parameters were reported. Therefore, white-matter degeneration does not represent an etiological factor for alcohol addiction. As it will be described in further chapters, our work differs substantially in both experimental design (Section 2.1) and analytical approaches (Sections 3.3 and 3.4). Gozzi and coworkers investigated the msP and Wistar rats from the innate aspects underlying higher alcohol consumption by assessment of multiple univariate MRI analysis; we aim to integrate multiple MRI modalities in a multivariate analytical scheme in a longitudinal experiment.

As described, alcohol use and abuse disorders affect the brain structures in multiple ways. Integrative statistical methods are lacking to handle overwhelming volume of data derived from complementary diagnostic tools. A set of methods originated in Machine Learning and Statistical 
Learning offers solutions to the huge amount of technically distant information characterizing brain diseases.

\subsection{Machine Learning in Brain Imaging}

Traditionally neuroimaging research has been performed through statistical inference or mass-univariate testing extracting features from one neuroimaging modality alone. Mass-univariate statistical testing performs iteratively statistical tests at each voxel making easy the understanding of brain structure and function. For example, task-related brain response is commonly investigated by temporal correlations of blood-oxygen-level-dependent (BOLD) signals and the experimental task time courses. Spatial representation of significant statistical effects enables us to identify taskrelated activations. Similar group statistical inferences can be performed in static brain images for MRI-derived parametric maps. One of the most popular analytical techniques to investigate brain structure is Voxel-Based morphometry which calculates voxel-enclosed tissue content (50). Finally, statistical inferences across groups in cross-sectional or several temporal stages in longitudinal studies are investigated. Similar analysis is performed in a wide variety of neuroimaging techniques such as DTI indices $(51,52)$; FDG-PET $(53,54)$ or resting-state fMRI-derived measures $(55-57)$.

An alternative to statistical approaches traditionally used in neuroimaging field is the Machine learning (ML), a branch of artificial intelligence, providing a huge group of algorithms of methods which allow accurately predict unseen data from empirically-developed classifiers integrating complementary information increasing diagnostic accuracies (58) or sensitivity to detect specific changes produced by a particular mental state. These techniques and algorithms concerned with the identification of patterns or regularities in data are known as supervised learning. For instance, machine learning based Multivariate-Pattern analysis has been successfully applied to functional MRI to detect patterns of BOLD activity associated to a particular cognitive or pathological mental state $(59,60)$. However, the most exploited application is to build automated and objective diagnostic tools predicting medical state. This idea has been widely applied in several neuroimaging studies in Alzheimer's disease (AD) (61-63), attention-deficit/hyperactivity disorder (ADHD) $(64,65)$, several forms of epilepsy $(66,67)$, dementia $(68)$ and many others psychiatric disorders. 
A potential application of $\mathrm{ML}$ approaches is the possibility of mapping pathological trajectories in patients. For instance, Luo and colleagues combined behavioral and PET predictors to predict treatment clinical outcomes (69) in a follow-up experimental design for cocaine addiction with successful predictive power. A recent work based on structural connectome (67) predicts with a $70 \%$ of accuracy the epilepsy clinical outcome after the surgery.

Despite the use of $M L$ techniques has enabled to identify strong predictors of drug initiation, maintenance and treatment response, few investigations have been carried on alcohol research in combination with neuroimaging biomarkers. By systematic search in PubMed database with the keywords machine learning, neuroimaging, and alcohol only 5 original works have been published so far (a review and a non-imaging study result also from the search). The oldest one dates back to year 2014 reflecting that predictive analysis is a hot topic in addiction research. Two of them aim to anticipate alcohol misuse in adolescents $(70,71)$. Both studies combine multimodal neuroimaging data, neuropsychological testing, and demographic and clinical information to identify biomarkers predicting future addictive behaviors. It is well-known that demographic status interacts with genetics leading drug misuses (72). Therefore, it is unclear from these multidimensional studies whether solely genetic factors can anticipate alcohol misuse. Seo and co-workers (73) aim to advance future relapse by purely clinical and neuroimaging predictors obtaining high accuracy rates of almost $80 \%$ which surpassed substantially purely clinical predictive models. Subthalamic nucleus functional connectivity discriminated alcohol drinkers with an accuracy of $60 \%$. Interestingly, classification in binge drinkers vs age-matched healthy group showed better performance (71.9\%) than discriminating alcohol dependents (64.7\%) reflecting probably that neuroadaptations are not permanent during abstinence periods (74). Other alcohol-related studies have used machine learning methods in the field of the genetics (75) or in the characterization of alcohol-related behaviors (76). Results obtained during the research presented in this dissertation have recently contributed to the available literature expanding the current knowledge by a data-driven and MRIderived definition of multimodal spatial signatures in a longitudinal experiment performed in msP rats (77). In the following, methodological framework and results will be presented. 


\section{Materials}

\subsection{Animal preparation}

A total of $18 \mathrm{msP}$ rats $(370-480 \mathrm{~g})$ and 10 Wistar rats $(350-400 \mathrm{~g})$ were used in these experiments. Experiments were performed at the Neuroscience Institute of the Spanish National Research Council (CSIC) - Miguel Hernández University. Rats were individually housed in transparent polycarbonate cages with bedding material, and a wooden stick and nesting material were given as enrichment. Rats had ad libitum access to food and water and were housed under controlled temperature $\left(22 \pm 2^{\circ} \mathrm{C}\right)$ and relative humidity $(55 \pm 10 \%)$ on a 12-hour light/dark cycle. The msP rats (imported from the breeding facility at the University of Camerino) were individually housed for 30 days with access to two drinking bottles, one containing water, and the other $10 \%$ EtOH diluted in water. Fluids consumption and animal weight were registered every 2-3 days concomitant with replacement of the bottles content. After one month of two-bottle free-choice drinking regime, the EtOH-containing bottle was removed. At this point msP animals $(n=18)$ were splitted in two groups. In the first group animals proceeded into abstinence for one additional week $(n=9)$. In the second group, the abstinent animals were medicated with $2.5 \mathrm{mg} / \mathrm{Kg} /$ day (i.p.) of naltrexone $(\mathrm{n}=9$ ). This dose has been demonstrated to reduce significantly voluntary EtOH consumption (17).

The timeline of the experimental design is depicted in the figure 2.1. 

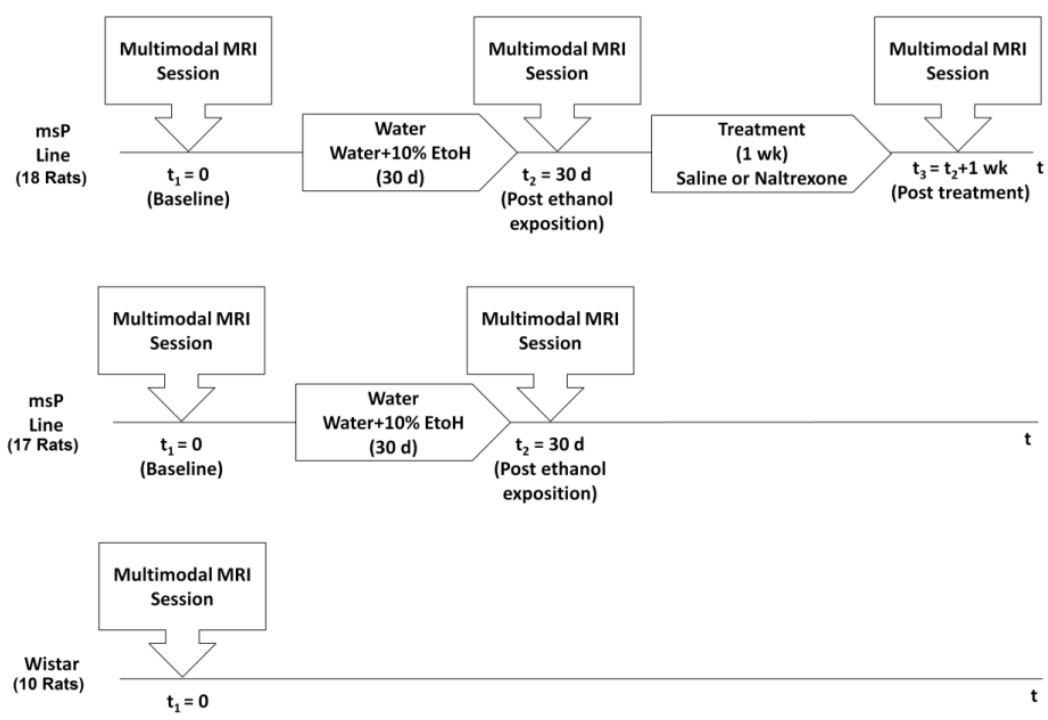

Figure 2.1 Timeline of the experimental design. Experimental design indicating the specific time points for treatment and imaging sessions performed in the longitudinal study of msP rats (upper diagram shows EtoH exposition and the middle panel its control) and the Wistar rats (lower diagram). Adapted from (77).

In the next section, behavioral results will be shown. These results are out of the main topic of this dissertation and therefore, not included in the main Results section.

All experiments were approved by the local authorities (IN-CSIC) and were performed in accordance with Spanish (law 32/2007) and European regulations (EU directive 86/609, EU decree 2001-486).

\section{Alcohol preference ratio}

During the period allocated for drinking in the two bottle free-choice paradigm, a time dependent pattern consumption assessed by the ratio of EtOH consumption and total fluids intake was found $\left(F_{(2.251,42.86)}=9.663, p=0.0001\right.$, one way repeated measures ANOVA). While the amount of alcohol is constant along the exposition $\left(F_{(2.705,45.99)}=0.7106, p=0.5369\right.$, one way repeated measures ANOVA), rats consumed less water at the end of the alcohol exposition $\left(F_{(1.436,24.41)}=8.597, p=0.0034\right.$, one way repeated measures ANOVA). An alternative is to calculate preference score defined as a ratio of taste solution intake to total fluid intake (ethanol solution plus water) (78). The daily fluid consumption and the ratio are shown in Figure 2.2A. A plateau in the preference of alcohol respect 
water is reached in the day 8 of alcohol exposure $\left(F_{(4.567,77.63)}=1.986, p=0.0961\right.$, one way repeated measures ANOVA). Using the $8^{\text {th }}$ day as the break point and leaving out the $5^{\text {th }}$ day, the alcohol preference is increased a $17.3 \%(t=3.94, p=0.0011)$. Average of immediate and at plateau alcohol choice over water is showed in Figure 2.2B. No significant Pearson correlation between transitory or initial preference and steady preference was found $(r=0.286, p=0.249)$.
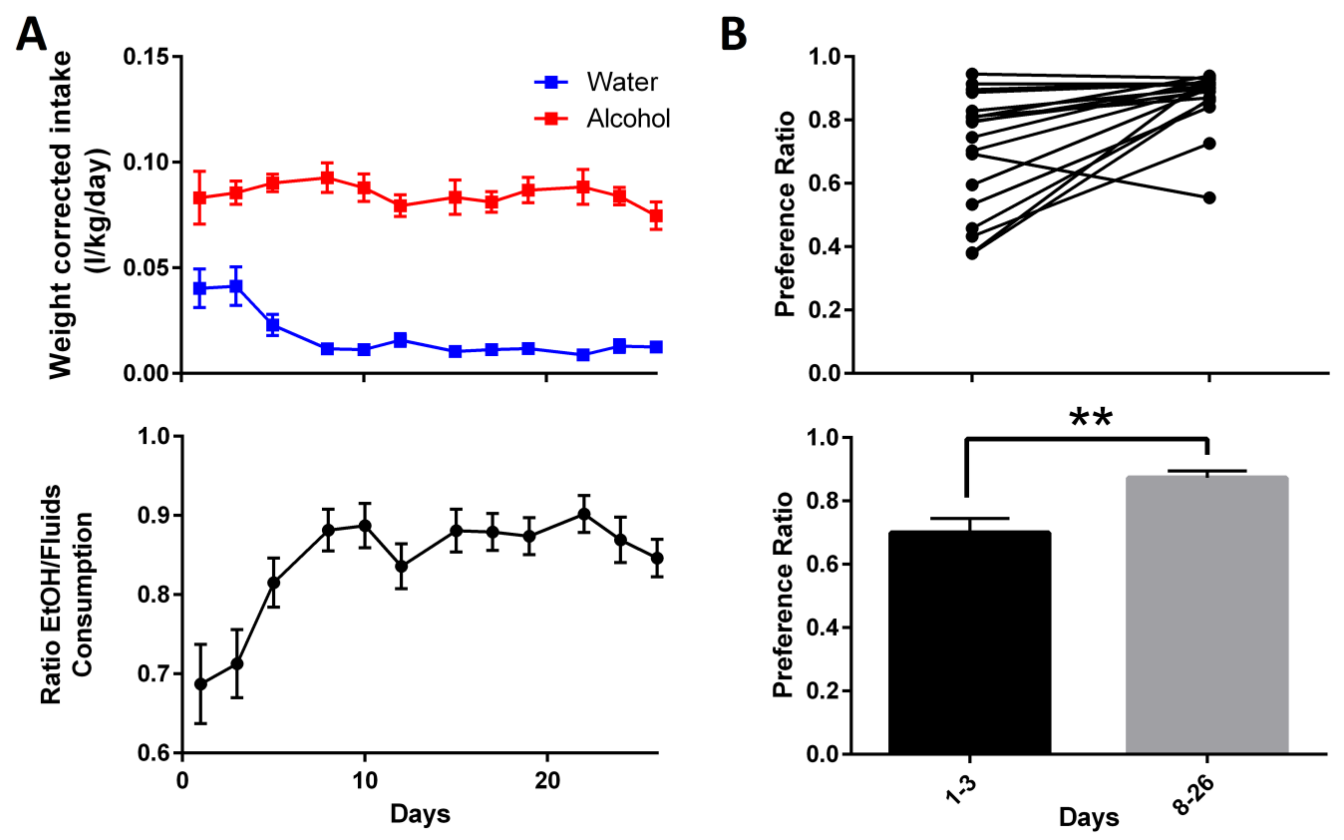

Figure 2.2 Ethanol consumption during one month of free-access. A) Alcohol and water consumption during the two bottles free-access paradigm (top) and alcohol preference ratio (down). (B) Individual changes in the preference before and after the breaking point (top) and mean comparisons (down). There were a significant increased alcohol preference ( $t=3.94, p=0.0011$, paired $t$ test). Data are expressed as mean $\pm S E M$.

\subsection{MRI Experiments}

Three multimodal imaging sessions were scheduled longitudinally in msP rats, before and after 1 month of alcohol drinking (session t1 and t2, respectively), and after one week of abstinence in the absence or presence of naltrexone medication ( $\mathrm{t} 3$ ) (see above and Fig. 5.1A). Imaging experiments were performed under anesthesia. Anesthesia was induced with $4-5 \%$ isoflurane in oxygen (0.8-1 L/min) and animals secured on a custom-made holding apparatus with a tooth bar and 
a nose cone. During scanning, the isoflurane concentration was maintained at $1.5 \%$, the body temperature was kept constant with a heating pad and physiologic parameters as oxygen saturation (SpO2), pulse distension, breathing and heart rate were monitored (MouseOx, Starr Life Sciences, Oakmont, US). A group of 10 Wistar rats were also scanned at t1 to assess inter-strain comparison.

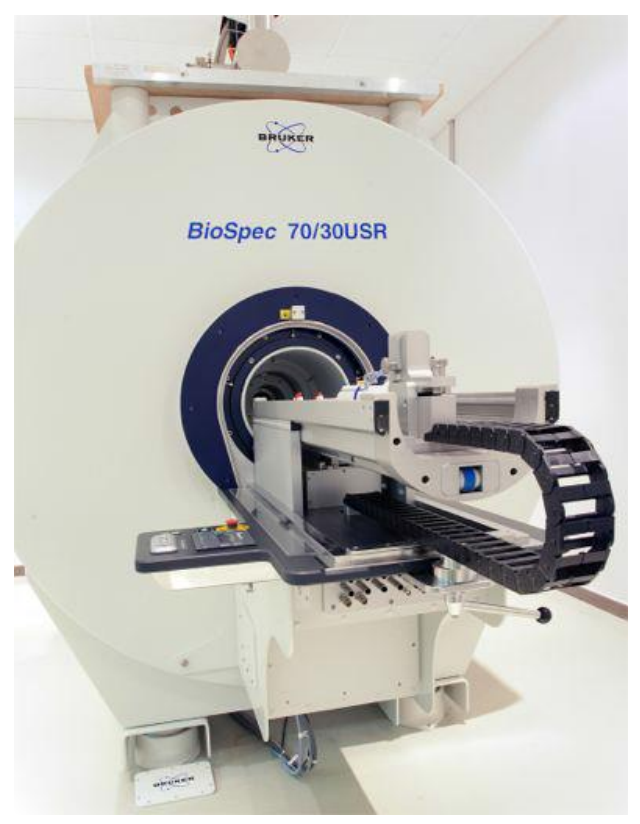

Figure 2.3 Magnetic Resonance scanner at Instituto de Neurociencias in Alicante in San Juan de Alicante (Spain). 7 T MRI scanner (Bruker, BioSpect 70/30, Ettlingen, Germany) used for the acquisition of the data presented in this Doctoral Thesis.

MRI experiments on rats were performed on a $7 \mathrm{~T}$ scanner (Bruker, BioSpect 70/30, Ettlingen, Germany) using a receive-only phase array coil with integrated combiner and preamplifier in combination with an actively detuned transmit-only resonator. Fourteen horizontal slices were planned for every subject (field of view $[\mathrm{FOV}]=32 \times 32 \mathrm{~mm}^{2}$, matrix size $=128 \times 128$, in-plane resolution $=0.25 \times 0.25 \mathrm{~mm}^{2}$, slice thickness $=1 \mathrm{~mm}$ ). All imaging modalities were acquired with the same geometry. Two superior and two inferior slices were removed in the relaxometry maps in order to reduce the MRI acquisition time.

Diffusion Tensor Imaging (DTI) data was acquired using an EchoPlanar Imaging diffusion sequence, with 30 uniformly distributed gradient directions, $b=670 \mathrm{~s} / \mathrm{mm}^{2}$, with four non-diffusion weighted images, repetition time $(T R)=4000 \mathrm{~ms}$, and echo time $(T E)=23 \mathrm{~ms}$. 
T2 map images were acquired using a multi-slice multi-echo sequence (TR = $6000 \mathrm{~ms}$, TE $=[12$ 24364860728496108120132144156168180192204216228240252264276288300312 324336348 360] ms).

T1 map images were acquired using a Rapid Acquisition with Relaxation Enhancement (RARE) sequence with variable repetition time (TE = $12.61 \mathrm{~ms}, \mathrm{TR}=\left[\begin{array}{l}155250400800160035006000\end{array}\right.$ ms).

T2-weighted images used for multimodal image coregistration were acquired using a Rapid Acquisition with Relaxation Enhancement (RARE) pulse sequence (TR $=3800 \mathrm{~ms}$, TE $=14 \mathrm{~ms}$, number of averages $=6, \mathrm{FOV}=32 \times 32 \mathrm{~mm}^{2}, 28$ horizontal slices of $0.5 \mathrm{~mm}$ thickness, and matrix size $=256 \times$ 256 , resulting in $0.125 \times 0.125 \times 0.5 \mathrm{~mm}^{3}$ voxel resolution) .

Residual artifacts might affect in a variety of ways the tensor reconstruction (79). As it has been also demonstrated, alcohol exposition increases the involuntary motor behavior in anesthetized subjects. We used the alignment parameters $([\alpha, \beta, \varphi, \mathrm{x}, \mathrm{y}, \mathrm{z}], 3$ rotation and 3 translation, respectively) of EPI dataset acquired during the scheduled MRI sessions (data not used in this work) to calculate the mean frame displacement (FD, Ec. 3.1) (80).

$$
F D_{i}=\left|\Delta d_{i x}\right|+\left|\Delta d_{i y}\right|+\left|\Delta d_{i z}\right|+\left|\Delta \alpha_{i}\right|+\left|\Delta \beta_{i}\right|+\left|\Delta \varphi_{i}\right|
$$

where

$$
\Delta d_{i x}=d_{(i-1) x}-d_{i x}
$$

similarly for the $\alpha, \beta, \varphi, y$ and $\mathrm{z}$. Rotational parameters were transformed to millimeters (radius of the sphere $5 \mathrm{~mm}$ matching rat brain dimensions). No differences in average unconscious movement were found at any experimental condition.

\subsection{Software and Computational Tools}

Image analysis was performed using Statistical Parametric Mapping Software (SPM, Wellcome 
Trust Centre for Neuroimaging, Institute of Neurology, University College London, London, United Kingdom), FMRIB Software Library v5.0 (FSL, Analysis Group, FMRIB, Oxford, United Kingdom) and a collection of in-house programs developed using MATLAB 7.1 (The Mathworks Inc., Natick, MA, USA) and the free software environment for statistical computing and graphics $R$ ( $R$ Foundation for Statistical Computing, Vienna, Austria). Other R packages used in the analysis incorporated the libraries Caret (https://topepo.github.io/caret), flowClust (81), and the package ggplot2 for visualizing some of the results (https://cran.r-project.org/web/packages/ggplot2). 


\section{Methods}

\subsection{Image pre-processing}

Image data was converted from Bruker MRI format to Analyze format. All rat images were resized by a factor of 10 (to fit human brain dimensions and use default settings of FSL and SPM tools). A voxel-by-voxel nonlinear least squares fitting of T1 and T2 to a monoexponential signal decay curve (Ec. 3.1 and 3.2 respectively) was performed at the relaxometry dataset. Parameters of the exponential signal decay were calculated and estimated by the non-linear least-squares fitting Trust-Region algorithm. T1 and T2 maps were corregistered to the T2-weighted images.

$$
\begin{gathered}
\mathrm{S}=\mathrm{S}_{0}\left(1-\mathrm{e}^{-\mathrm{TR} / \mathrm{T} 1}\right) \\
\mathrm{S}=\mathrm{S}_{0} \mathrm{e}^{-\mathrm{TE} / \mathrm{T} 2}
\end{gathered}
$$

DTI data were corrected for motion and eddy current artifacts and local diffusion tensor were fitted using FSL. From the diffusion tensor components, fractional anisotropy (FA) and mean diffusivity maps (MD) were determined.

Structural images were used to create the group template and parametric maps using the SPM Diffeomorphic Anatomical Registration through Exponentiated Algebra or DARTEL procedure (82). Detailed procedure is described in the following section. Briefly, for each subject, the structural image was first segmented and gray- and white-matter tissues collected to prepare the group template. Afterwards, an averaged- gray matter, white matter and cerebrospinal fluid segments were non-linear normalized to the stereotaxic coordinates (83) and used as tissue probability maps in the Unified Segmentation approach implemented in SPM (84). Gray and white matter segments were non-linearly warped and modulated to preserve the local volume of the tissue. FA MD, T1, and T2 maps were also non-linearly normalized and resampled. In order to reduce misalignments artifacts between MRI modalities and increase the performance, normalized parametric maps were smoothed with a $6 \mathrm{~mm}$ isotropic Gaussian Kernel filter (85). Smoothing approximates the data to be normally distributed, a requirement of Gaussian random field theory (GRF) to correct for type I 
error.

\section{Tissue Probability maps and template creation}

Healthy brains are generally formed for three broad tissue types: grey matter (GM), white matter (WM) and cerebro-spinal fluid (CSF). The tissue classification employed in this work has been carried out on SPM software. The tissue classification algorithm implemented at SPM lead to assign to each voxel a probability of belonging to a particular tissue class based only in its intensity and a prior knowledge of the spatial distributions of these tissues in the form of prior probability maps or indistinctly called tissue probability maps.

Several papers have been focused on the construction of highly detailed rodent high field MRI templates for a variety of neuroimaging applications such as robust morphometric analysis and DTI (86-89). Nevertheless, none of them enjoyed great popularity among the scientific community and the tendency is to create a set of template and tissue probability maps for each application or experiment. Only the proposed by Schwarz (83) has enjoyed of popularity and has been widely used in several works, although unfortunately these set of templates do not include an GM,WM,CSF prior information.

The probabilistic segmentation framework implemented by SPM leads to unify a tissue classification approach and a registration with a template through a circular procedure which includes parameters that account for tissue classification, intensity inhomogeneity correction and registration.

From msP rats, a group template and tissue classification maps were created by using the SPM and FSL software. Biedermann and coworkers proposed an approach to create the template and slightly modifications in some steps have been included (90). T2 weighted structural data were realigned using a rigid transformation. Realigned images were rescaled by a factor of 0.5 in the anterior-posterior direction in order to make the brain more spherical and to raise the accuracy of skull-stripping (BET, (91)). Then, BET output was reversed to the original shape. Due to the lack of prior knowledge of tissue probability, same probability of each voxel to belong to each tissue is assumed. Namely, mask outputted from BET was used as a prior knowledge in the SPM probabilistic segmentation framework. As the segmentation approach is based on a mixture of Gaussian, one 
component per tissue class and 4 Gaussians for noise and non-brain tissue were used to fit to intensity histogram. Spatial probability maps were replaced with the mask of the whole brain in the SPM routine for the first run. SPM was run again replacing the spatial probability maps with the output of the first run. In order to improve the accuracy of skull-stripping, a spatial probability map of the brain tissue was created from the sum of the tissue probability maps. A new run was performed as before but using a brain probability map rather than BET output. Once all the images were segmented and assigned to each tissue class, a group template for the brain tissues was created. GM and WM segments were collected together in order to create an individual flow field using the SPM Diffeomorphic Anatomical Registration through Exponentiated Algebra (82). It models the non-linear spatial transformation matching the group template. All the tissue segments and anatomical image were warped to the group space using the flow field previously calculated in order to create a mean tissue probability map and an anatomical template of the group. Finally, data were non-linearly transformed to the reference anatomical space using the Paxinos and Watson Atlas (92). Templates and tissue probability maps are shown in Figure 3.1. 


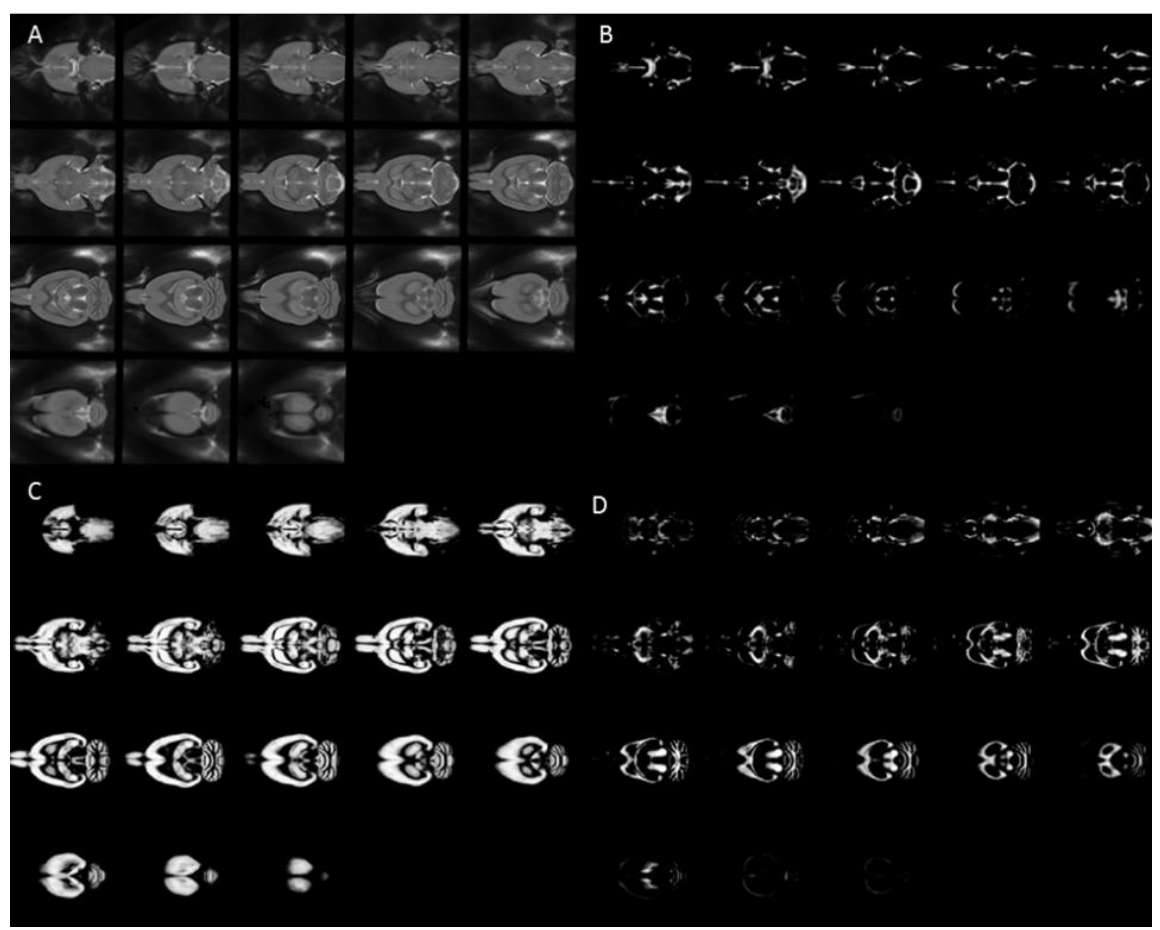

Figure 3.1 Template and tissue probability maps derived from MRI. Ventral to dorsal slices of the average group template (a), cerebro spinal fluid (B), grey (C) and white (D) matter probability map used to estimate grey matter and white matter maps. 


\subsection{Introduction to Machine Learning algorithms}

In this section the ML approach used across the entire work is introduced. The first two subsections are devoted to present the general overview of two of the main concepts used in $\mathrm{ML}$ applications: classification and feature selection. Validation and common pitfalls are presented in the third subsection. A fourth part is devoted to the implemented classification framework in this research.

\subsubsection{Support Vector Machine classification}

As introduced in Chapter 1 , in biomedical and biological data analysis such as genetics or neuroimaging studies, an important task is to predict a categorical response from observed characteristics. For this purpose, two main families of algorithms exist: unsupervised and supervised learning.

Unsupervised analysis or unsupervised clustering analysis is an important and powerful tool to identify subgroups in the sample. The goal of unsupervised learned approaches is to identify underlying data structure or probability distribution generating such data patterns. Unsupervised learning problems can be grouped into clustering and association problems. Association problems consist in solving the problem $A \rightarrow B$, where $A$ and $B$ are two disjoint item sets. A simple example of association problems is to identify the rules that describe why people who buy $A$ also tend to buy $B$. Clustering is the process of portioning a set of patterns into clusters or groups by minimizing and maximizing similarities intraclusters and interclusters respectively. A clustering technique assessed during the research in the context of this dissertation is detailed in the Section 3.3.

In this research we mainly focus in the classification problem of several experimental conditions, namely, determine the categorical response (class) of instances that are characterized by variables (features or predictors are used indistinctly). This is the main goal of supervised learning approaches or classifiers.

Classifiers can be also grouped as generative or discriminative. Generative classifiers learn a model of the joint probability $p(x, y)$ of inputs and outputs and use Bayes rules to calculate $p(y \mid x)$. In contrast, discriminative classifiers solve the problem $p(y \mid x)$ without solving an intermediate step. 
In this work, we implemented a discriminative classifier based on Support Vector Machines (SVM).

As introduced previously, a classifier is a set of rules or functions $f$ that partitions a set of objects $x_{i} \in \mathbb{R}^{n}, i=1, \ldots, l$ into classes $y_{i} \in\{-1,1\}, i=1, \ldots, l$ for the binary classification. The classifying task can be simplified as a task of calculating a separating hyperplane that distinguishes two classes (Eq. 3.1).

$$
f\left(x_{i}\right)=\operatorname{sign}\left(\omega x_{i}+b\right)
$$

The hyperplane $H$ is a linear function that is capable of separating the training data accurately by minimizing some loss function (93). The hyperplane is described as (Eq. 3.2):

$$
H: \omega^{T} x_{i}+b=0
$$

Where $\omega$ is the learning weight vector perpendicular to the hyperplane separating classes, $b$ an offset, and $x_{i}$ the observed data. Once the hyperplane or classification rule is learned from the training data, the model can be utilized as a decision function to categorize new samples. However, a model that works correctly with the training data can fail predicting unseen samples if a local optimum solution is achieved instead of a global solution (convex optimization). To overcome this limitation, the Support Vector Machines (SVM) has emerged as a powerful tool for statistical pattern recognition becoming one of the most popular algorithm in classification tasks $(94,95)$. SVM algorithms find the optimal model maximizing the distance from the separating hyperplane and the closest training sample. This distance is called margin and the generalization of the model improves as the margin increases. Another of the most important benefits that makes SVM an excellent tool in the field of supervised pattern recognition methods is the ability to transform nonlinear problems into linear and thus simplifies the model from the fact that most linear methods only require the computation of dot products. This is known as the kernel trick. Commonly used kernels are for instance Gaussian, Polynomial and sigmoid. Kernel-trick is not exclusive of SVM algorithm. Another kernel-based learning approaches are Kernel Fisher discriminant or Kernel Principal Components $(96,97)$. 


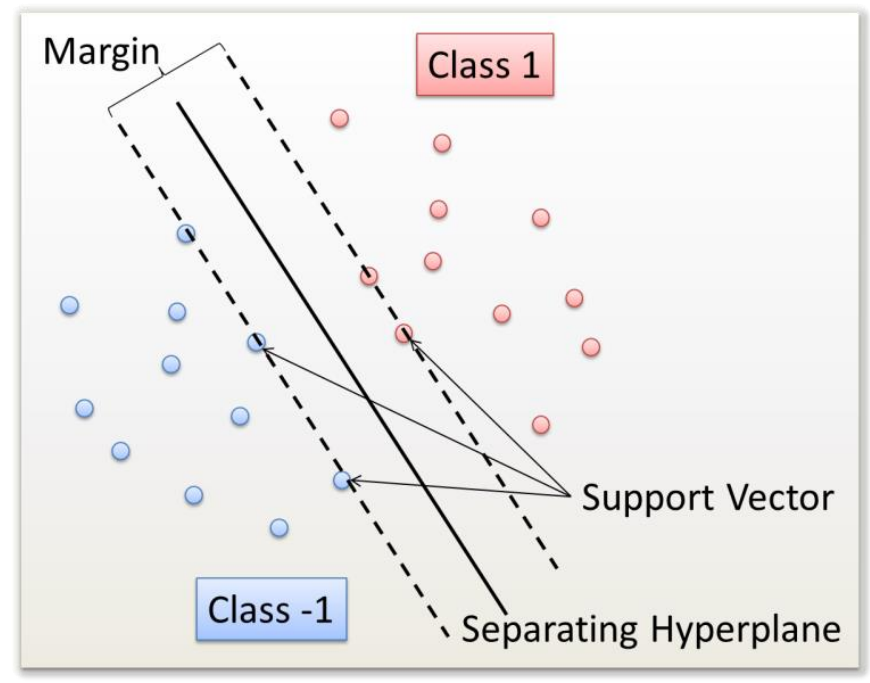

Figure 3.2 SVM classification. A hyperplane is used to discriminate both classes [-1, 1$]$.

As introduced above, the goal of the approach is to find the optimal hyperplane with the maximal separation between two classes (Fig. 3.2). The margin measured perpendicularly to the hyperplane is $1 /\|\omega\|^{2}$. To optiimize the SVM model consist in maximizing $1 /\|\omega\|^{2}$, i.e. minimizing $\|\omega\|$ in the equation. The SVM formulation is traditionally extended by slack variables to allow a certain degree of misclassification of the data $(\xi, C)$ (Eq. 3.5).

$$
\min _{\omega, b, \xi} \frac{1}{2} \omega^{T} \omega+C \sum_{i=1}^{l} \xi_{i}
$$

subject to $y_{i}\left(\omega^{T} x_{i}+b\right) \geq 1-\xi_{i}$ and $\xi_{i} \geq 0, i=1, \ldots, l$.

The above problem (Ec. 3.5) is usually solved by the dual problem:

$$
\min _{\alpha} \frac{1}{2} \alpha^{T} Q \alpha-e^{T} \alpha
$$

subject to $y^{T} \alpha=0,0 \leq \alpha_{i} \leq 1, i=1, \ldots, l$. $e$ is a vector of all ones and $\mathrm{Q}$ is a $l \times l$ positive semidefinite matrix with $Q_{i j}=y_{i} y_{j} x_{i}^{T} x_{j}$. After solving the problem (Eq. 3.6), the optimal $\omega$ satisfies 


$$
\omega^{*}=\sum_{i=1}^{l} y_{i} \alpha_{i} x_{i}
$$

And the offset $b$ is given by solving:

$$
y_{i}\left(x_{i} \omega^{*}+b\right)=1
$$

Then, we can rewrite the classification problem (Eq. 3.3) as the decision function

$$
f(x)=\operatorname{sign}\left(\omega^{*} x+b^{*}\right)=\operatorname{sign}\left(\sum_{i=1}^{l} y_{i} \alpha_{i} x_{\boldsymbol{i}} x+b^{*}\right)
$$

Note that in the above formulation no mapping into higher-dimensional space $\phi$ has been used. If a non-linear kernel $K\left(\boldsymbol{x}_{\boldsymbol{i}}, \boldsymbol{x}\right)=\phi\left(\boldsymbol{x}_{\boldsymbol{i}}\right) \phi(\boldsymbol{x})$ is used to solve nonlinear problems the decision function can be rewritten as:

$$
f(x)=\operatorname{sign}\left(\omega^{*} \phi(\boldsymbol{x})+b^{*}\right)=\operatorname{sign}\left(\sum_{i=1}^{l} y_{i} \alpha_{i} K\left(\boldsymbol{x}_{\boldsymbol{i}}, \boldsymbol{x}\right)+b^{*}\right)
$$

In this work we adopted linear kernel function $K\left(\boldsymbol{x}_{\boldsymbol{i}}, \boldsymbol{x}_{\boldsymbol{j}}\right)=1+\boldsymbol{x}_{\boldsymbol{i}}^{T} \boldsymbol{x}_{\boldsymbol{j}}$ resulting in a good performance.

One of the disadvantages of SVM classifiers is that it does not provide posterior probabilities and it is limited to provide decision values in the range of $[-\infty, \infty]$. To overcome this limitations, Platt scaling transforms the outcome of a classification model into prediction probabilities by fitting a logistic regression (98). 


\subsubsection{Feature Extraction and Selection with Random Forest Algorithm}

There are several methodologies or algorithms to define the set of rules determining the class with the greatest possible accuracy. However, the high dimensionality of feature space $\mathrm{p}$ and the low number of samples $\mathrm{n}$ are common characteristics in many of the biomedical machine learning applications ( $\mathrm{n}<<\mathrm{p}$ or so-called small $n$, large $p$ problem). Therefore, the identification of important predictors and discarding irrelevant ones is a critical step to (i) avoid overfitting (loss of generality of the prediction model) $(99,100)$, (ii) reducing the complexity of computation for prediction; (iii) removing information redundancy (cost savings) (101, 102); (iv) easing interpretation and (v) reducing the effect of the so-called curse of dimensionality $(103,104)$ This task is known as Feature Selection and is often used used before prediction models (regression or classification). The selection of features with higher predictive power is not a trivial task. Brute-force algorithms evaluating every possible subset of features are computationally expensive growing exponentially with the number of predictors. When exact techniques are not available because a large dimensionality of feature space, heuristic approaches can be used to rank predictors according to some predictive contributive power $(105,106)$.

Three main classes of feature selection techniques coexist: internal variable selection, filtering and wrapped methods.

Internal variables selection methods perform variable selection in the training or learning process. An example of tool implementing internal variable selection is the decision trees (DT). An intrinsic characteristic of the decision trees (DT) is to build decision rules according to some selected attribute (107). The selection of the variable is chosen by maximizing node purity of its descendant nodes. Next sections will elaborate this aspect.

Variable filtering consists in removing some variables according to a chosen statistic defined on the random variable over multiple data groups. Therefore, filters are univariate statistics including diverse discriminative power such as t-statistics, F-statistics, Fischer's discriminant ratio, maximum entropy, etc. 
Wrapped methods find the small subset of predictors that achieves the maximal performance model in terms of predictability. The search algorithm includes stepwise (forward or backward or both) variable inclusion by maximizing model performance.

In this research, we focus in wrapped methods by identifying a subset of important predictors. Different approaches can be used to rank features. The basis of such approaches is to calculate some measure of variable importance. Univariate tests over one feature can be performed ranking features according its discriminative power. In contrast, multivariate approaches consider the entire set or a subset of features simultaneously. An appropriate widely used approach for prediction and variable selection is the nonlinear and nonparametric random forest (RF) method. In the following, a more detailed introduction is provided.

Random forest is a popular and very efficient algorithm based on model aggregation ideas for regression and classification problems which belongs to the family of ensemble methods. From (108), RF is described as a "combination of tree predictors such that each tree depends on the values of a random vector sampled independently and with the same distribution for all trees in the forest". RF algorithm also possesses some appealing properties making it well-suited for prediction and feature selection estimation: (i) it shows good performance in small $n$, large p problems, (ii) it performs internal feature selection, (iii) it can handle nonlinearities and correlations between features, (iv) it is applicable for both binary and multicategory classification tasks, (v) it is applicable for continuous and categorical predictors, and (vi) it does not require much fine-tuning of parameters to achieve good performance.

First of all, let us introduce the decision tree (DT). DT is a non-parametric supervised learning method to predict a response variable from observed data features. DT consists in a set of splitting nodes which sends the data to the right and left leaf by thresholding a single observed variable (decision rule). In general, a set of decision rules form a decision tree. In the figure below, an instance of a binary decision tree is depicted. The set of decision rules $X_{i}>t_{j}, X_{i} \leq t_{j}$ define the decision path to classify a new sample (class 1 or class 2). An example is depicted in Figure 3.3. 


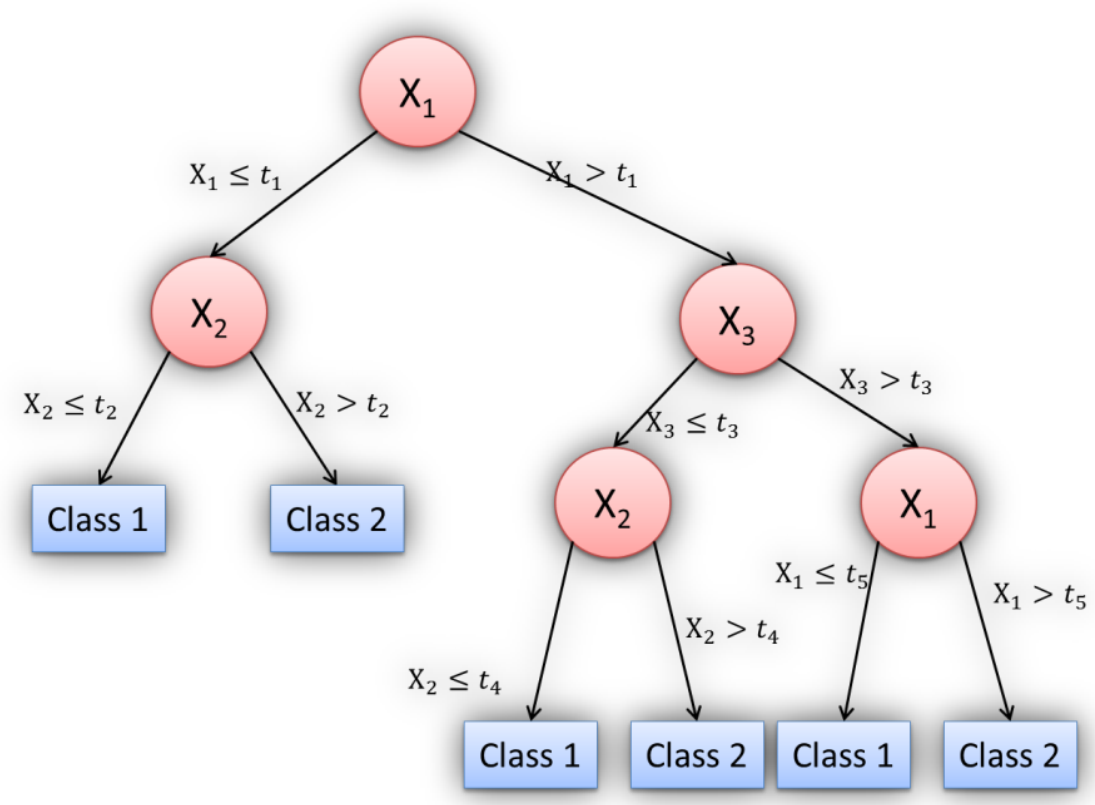

Figure 3.3 Hypothetical decision tree to identify classes

Specifically, a tree is built from a learning data set using a recursive procedure which identifies at each node $\tau$, the split $X_{i}=t_{j}$ which minimizes some impurity measurement. In $R F$, the Gini Index is often used to measure the node purity

$$
i(\tau)=1-\sum_{k=1}^{K} p_{k}{ }^{2}
$$

where $p_{k}$ is the fraction of the $n$ samples for each class in one of the subnodes $\tau^{L}$ and $\tau^{R}$ (left and right leaf respectively) with the respective fractions $p_{L}=\frac{n_{L}}{n}$ and $p_{R}=\frac{n_{R}}{n}$. Then, the mean decrease of the Gini Index at a node of the tree is defined as

$$
\Delta i(\tau)=i(\tau)-p_{R} i\left(\tau^{R}\right)-p_{L} i\left(\tau^{L}\right)
$$

As stated by Breiman, the principle of random forest is to combine many weak binary decision trees to make stable predictions. Each tree $\mathrm{T}$ in the forest (with ntree trees) is built using a bootstrap 
sample of the data, and at each split the candidate set of variables is a random subset of the variables (mtry). As mentioned above Random Forest algorithm assigns implicitly importance to each predictor, the Gini importance. The Gini importance of a variable is the sum of all the decrease in Gini impurity index for a given predictor normalized by the number of trees in the forest (3.13).

$$
I_{G}\left(x_{k}\right)=\frac{1}{n_{\text {tree }}} \sum_{t=1}^{n_{\text {tree }}} \sum_{\tau} \Delta i_{x_{k}}(\tau, t)
$$

In addition to mean decrease Gini as measure of importance, a different measure of importance has been proposed based on the decrease when the variable used in the decision rule is permuted in the out-of-bag set, the mean decrease accuracy. Both measurements have shown to be biased in some extent (109). However, the stability of both measurements is still under discussion. We performed preliminary explorations demonstrating a good agreement in both estimated variable importance measures (correlations above 0.85 , results not shown). For that reason, and that mean decrease accuracy implementation often results in negative importance weights hard to interpret, we adopted the Gini importance as measure to rank the features in this research.

\subsubsection{Validation and possible bias of the predictions}

In the previous sections, approaches for learning the decision rules for predicting unseen data have been introduced in detail. A common practice to evaluate the validity of the prediction models and the error generalization are the resampling methods. Specifically in this research we used k-fold cross-validation (CV) methods which splits data sets in two unbalanced sets: training and testing sets. Training and testing set is constructed by randomly partitioning the data in $\mathrm{k}$ folds and choosing one of the folds for testing and the remaining k-1 for training. Prediction model are then fitted to samples from training set and evaluated in the testing set. This produces $k$ models that are all trained on different subsets of the initial dataset and where each of the subsets have been used as the test partition once resulting in k error estimations. The cross-validation estimated accuracy is computed as the average across the $\mathrm{k}$ models. Particular cases of cross-validation is the leave-oneout cross-validation (LOOCV) with $k$ equals the number of samples and stratified $k$-fold cross- 
validation (partitioning is not entirely random). We adopted the latter one for evaluating classification models with longitudinal outcomes. In this case the folds were subject-wise constructed meaning that the training or testing set includes all longitudinal samples of subjects randomly selected.

In order to quantify the predictive power of the model, accuracy (3.12), sensitivity (3.13) and specificity (3.14) are the terms commonly used in binary classification. In general, sensitivity and specificity indicate how well the model predicts each of the categories, whereas accuracy measures how well the test predicts both categories. Mathematically, these measures can be quantified using the following equations:

$$
\begin{gathered}
\text { Accuracy }=\frac{T P+T N}{T P+T N+F P+F N} \\
\text { Sensitivity }=\frac{T P}{T P+F N} \\
\text { Specificity }=\frac{T N}{T N+F P}
\end{gathered}
$$

where TP, TN, FP, FN represents the number of true positives, true negatives, false positives and false negatives respectively. Sensitivity and specificity can be interchangeable according how positive class is defined. As well, Receiver-operating characteristic (ROC) provides a way to summarize the accuracy of the prediction (true-positive rate vs false-positive rate) associated at different cutting values. The area under curve the ROC (AUC-ROC) is a useful summary of the curve whereas AUC-ROC $\approx 1$ indicates excellent classification and AUC-ROC $\approx 0.5$ classification by chance and therefore no classification utility. In general, a tradeoff between sensitivity and specificity is desirable, however in some medical applications for early detection and treatment of pathologies, higher sensitivity, rather than higher specificity would be desirable.

Special considerations are required when supervised feature selection or model hyperparameter tuning precedes the data-driven classification. Specifically, a significant bias in the evaluation of the generalization of the prediction can be introduced when both training and testing set in k-fold cross validation are included are used for supervised feature selection (110). As evident, 
the testing set leaks partly the training phase limiting the generalization of the result and resulting in an optimistic and unrealistic estimation of the error. In the next section, we introduce two different frameworks to evaluate the introduced bias.

\subsubsection{Wrapped SVM classification according RF variable importance}

Several ways to combine multisource data sets in a $\mathrm{ML}$ scheme are currently used. For instance, multiple kernel learning (111) whereas multiple predictive models could be constructed from individual neuroimaging sources and then combining them to form a single kernel (112). A practical alternative is to concatenate multisource predictors in a single feature vector as input for predictive models. In this work we propose the use of RF to rank the multimodal features altogether according its importance and subsequently, to iteratively and forwardly train SVM models with different sizes of variable subsets. Here are the generic steps of the procedure:

1. Partition the data for k-fold cross-validation.

2. On each CV training set, RF is trained using all the variables (note that RF algorithm randomly selects a subset of variables and samples) to produce a ranking according their Gini Importance (Record the CV training set importances).

3. Train SVM learning machine with the most important feature and the training test and evaluate the performance of the classification in the testing set. Repeat this until all the predictors are used.

4. Average the performance across all the k-folds to obtain performance as function of the number of predictors $p$.

5. Select $p^{*}$ that maximizes the accuracy vs. number of variables.

It is important to note the fact that different folds will produce a different feature ranking given the high variance of variable importance in small data sets and therefore, will make hard to identify subsets of features with high contributions to the classification. To overcome this limitation, random forest can run on the entire data set to provide a single ranking. However, as mentioned above, this modification results in a suboptimal design of the analytical pipeline which may overfit 
the results and therefore reduces the generalization of the approach. For this we have designed two frameworks (Figure 3.4 and Algorithm 3.1 and Algorithm 3.2). In order to quantify the introduced overestimation, we deliberately biased the approach by including the training sets as part of the features selection step (Figure 3.4B and Algorithm 3.1). It is worth to emphasize that implementation 1 finds the best dimension of the model. On the contrary, the implementation 2 finds the subset of variables for making accurate predictions.

Both schemes rank features according RF importances and perform classification based in SVM with linear kernels. Note that different algorithms might be implemented into the classification scheme. Nevertheless, both RF and SVM have been proven to perform accurately in classification based on neuroimaging biomarkers.

A

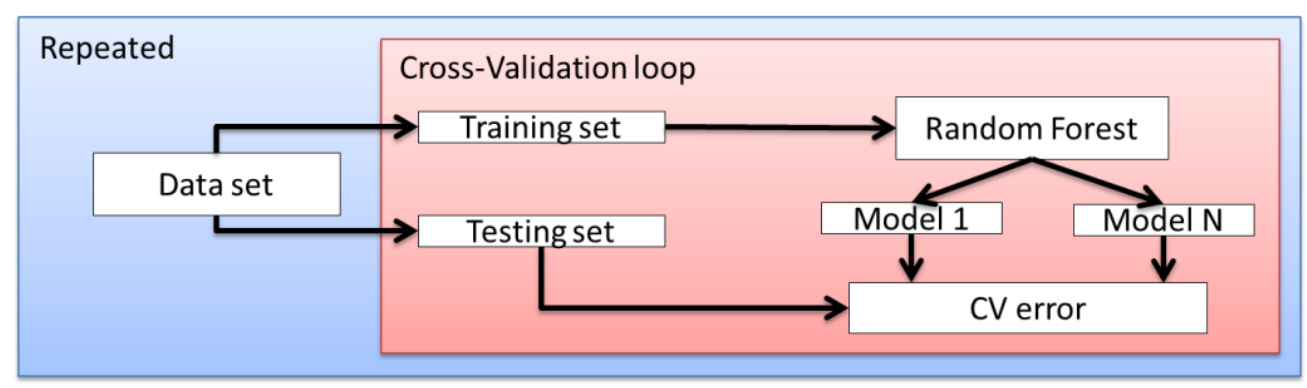

B

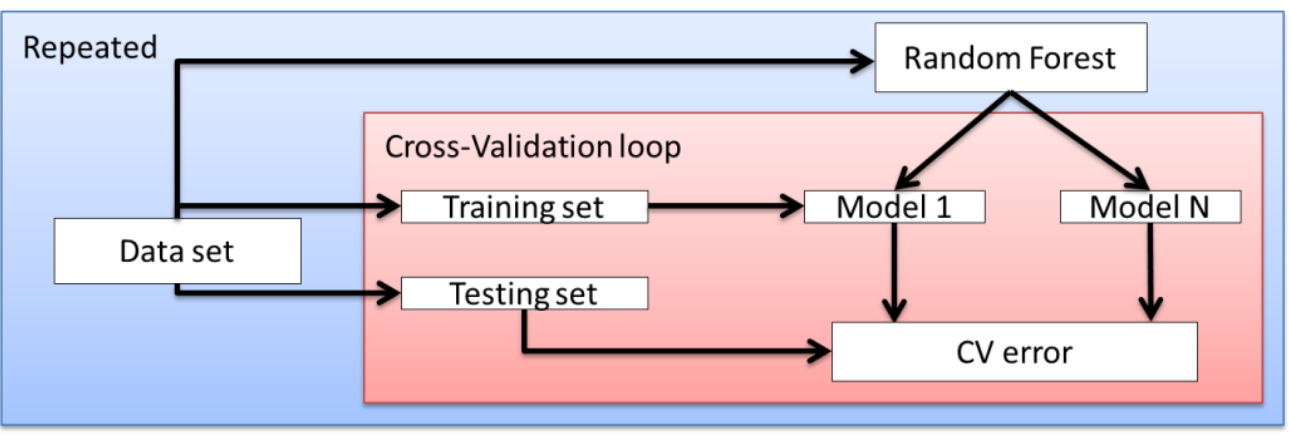

Figure 3.4 Classification schemes implemented. (A) Optimal framework avoiding risk of overfitting and (B) framework with high risk of producing biased predictions. Detailed description of the algorithms is provided in Algorithm 5.1 and 5.2. 


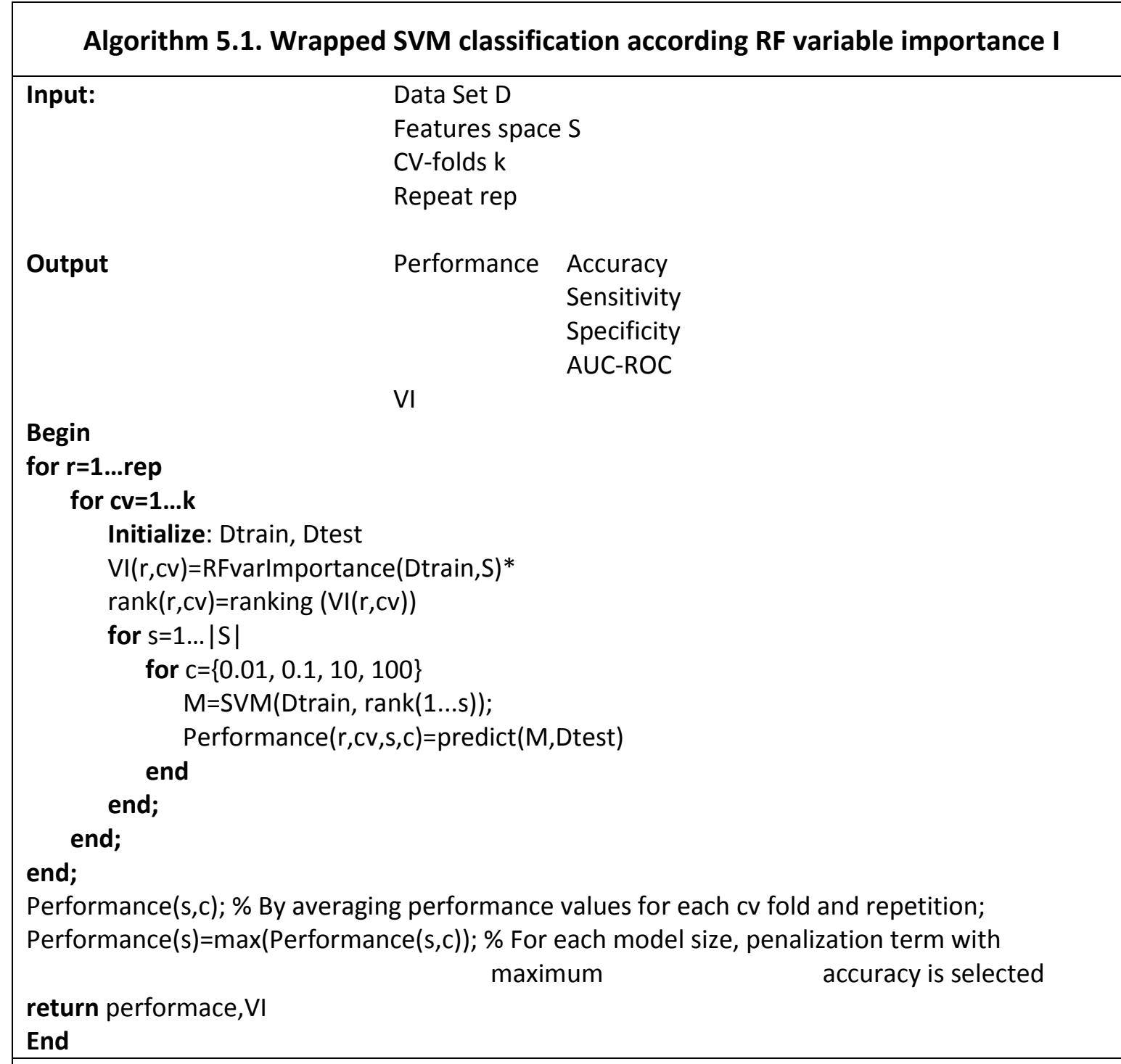

Algorithm 5.2. Wrapped SVM classification according RF variable importance II

\begin{tabular}{|c|c|c|}
\hline \multirow[t]{4}{*}{ Input: } & \multicolumn{2}{|l|}{ Data Set D } \\
\hline & \multicolumn{2}{|c|}{ Features space S } \\
\hline & \multicolumn{2}{|l|}{ CV-folds k } \\
\hline & \multicolumn{2}{|l|}{ Repeat rep } \\
\hline \multirow[t]{5}{*}{ Output } & Performance & Accuracy \\
\hline & & Sensitivity \\
\hline & & Specificity \\
\hline & & AUC-ROC \\
\hline & \multicolumn{2}{|l|}{$\mathrm{VI}$} \\
\hline
\end{tabular}




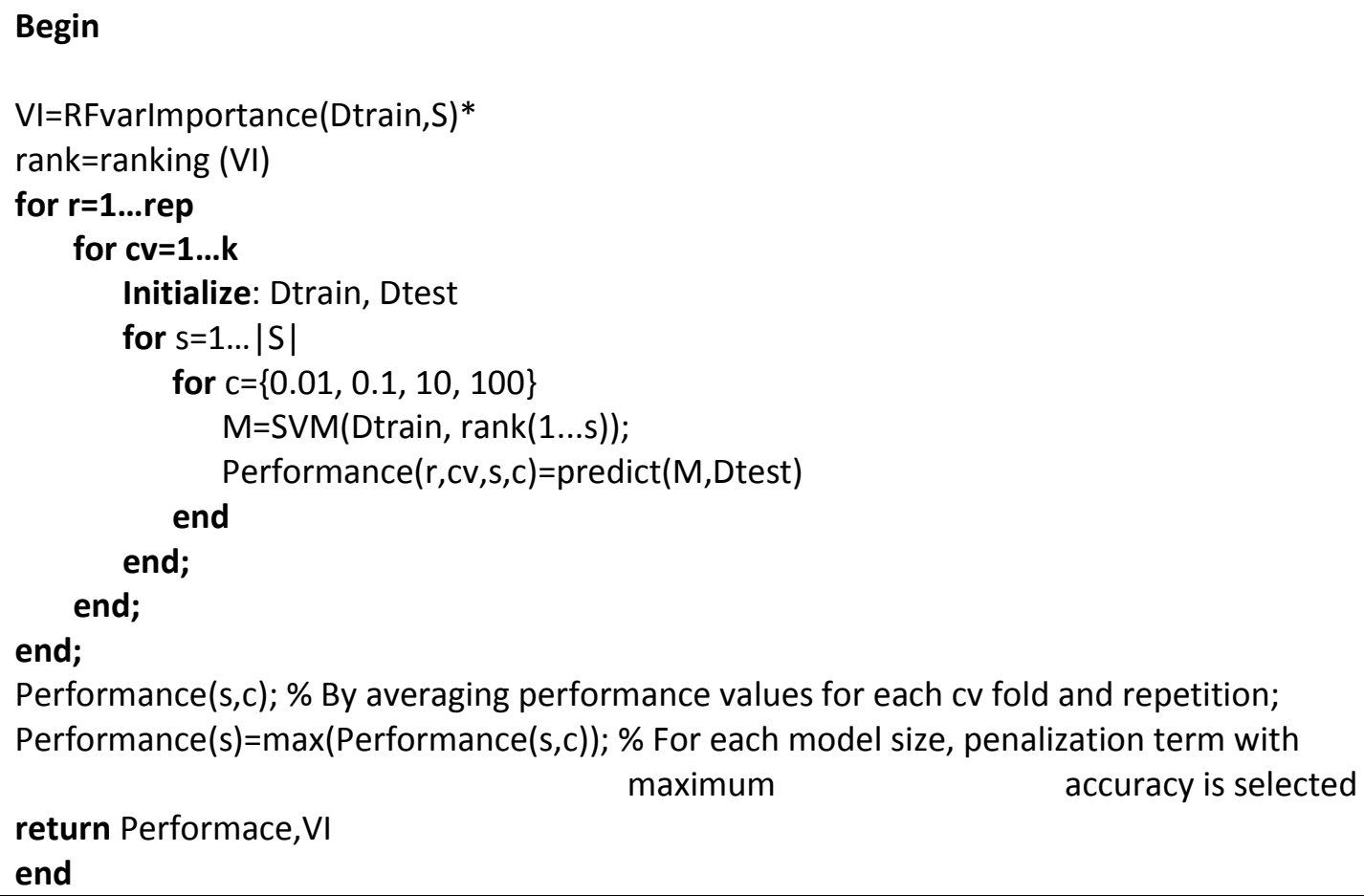




\subsection{Multimodal magnetic resonance imaging as a tool for identifying the different stages of alcohol addiction}

Work in this chapter has been partly published in (77):

Cosa, A., Moreno, A., Pacheco-Torres, J., Ciccocioppo, R., Hyytia, P., Sommer, W. H., Moratal, D. \& Canals, S. 2016. Multi-modal MRI classifiers identify excessive alcohol consumption and treatment effects in the brain. Addiction Biology 2016 Jun 8. [Epub ahead of print].

In this section, we propose a framework to explore the simultaneous combination of multiple parameters extracted from different MRI modalities such as mean water diffusivity (MD), fractional anisotropy (FA), T1 and T2 relaxometry times, as well as descriptors of the statistical distribution of those parameters, to build multimodal imaging signatures of disease stage (MSDs). Note that structural GMv and WMv maps were not used in the current part. More specifically, we propose that multi-parametric brain imaging combined with probabilistic tissue segmentation and machine learning techniques may provide an accurate readout of brain networks evolution after chronic alcohol exposure. In a scenario in which disease stage is not defined by time passed but the individual history of drinking/abstinence cycles, neuropsychiatric evaluations assisted by MISD could be valuable for accurate diagnosis and prognosis.

\subsubsection{Multimodal ROI (mROI) definition}

Finite mixture models are a mature method for probabilistic clustering and density estimation. The idea behind this method is that the probability distribution of the data $\boldsymbol{x}$ can be described by a weighted sum of $k$ probability functions:

$$
p(\boldsymbol{x})=\sum_{i=1}^{k} w_{i} f\left(\boldsymbol{x} \mid \theta_{i}\right)
$$

where $w_{i}$ are weighting factors or mixing proportions with $\sum_{i=1}^{k} w_{i}=1$ and $f\left(\boldsymbol{x} \mid \theta_{i}\right)$ is the density function component associated to the parameter $\theta_{i}$. As will be described below, Gaussian or $\mathrm{t}$ Student distribution forms the mixture. 


$$
f\left(\boldsymbol{x} \mid \mu_{i}, \Sigma_{i}\right)=\frac{1}{(2 \pi)^{D / 2}|\Sigma|^{1 / 2}} e^{\frac{1}{2}\left(x-\mu_{i}\right)^{T} \Sigma_{i}{ }^{-1}\left(x-\mu_{i}\right)}
$$
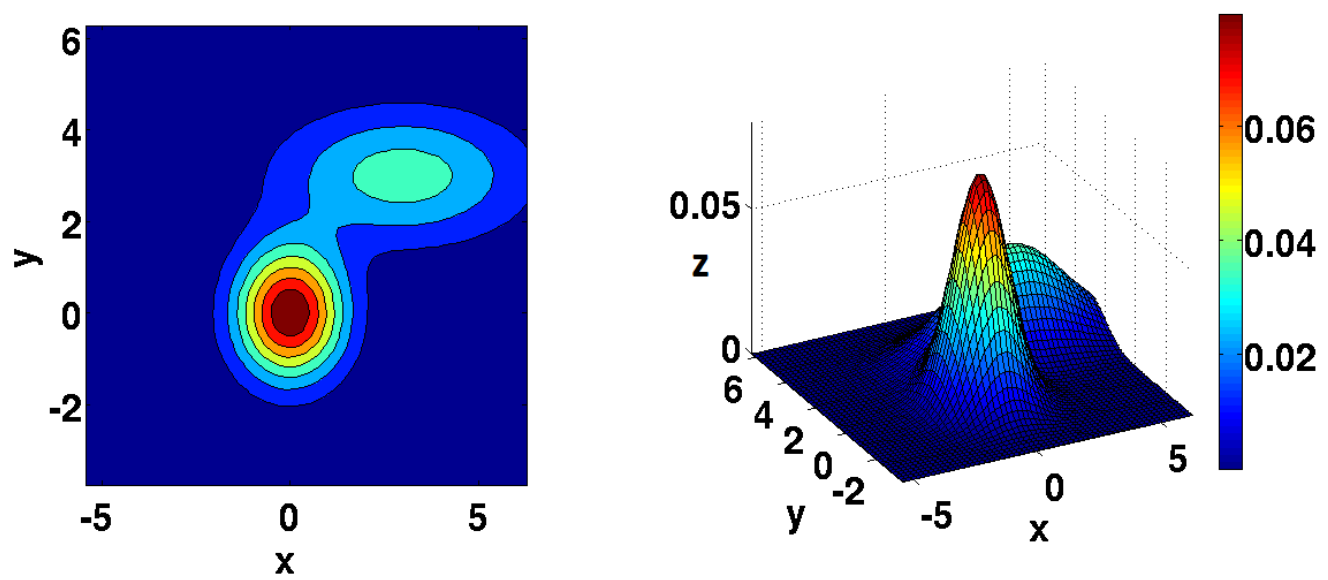

Figure 3.5 Schematic representation of probability distribution of mixture of 2 Gaussian components in 2D (left) and 3D (right).

Figure 3.5 shows an example of probability distribution of mixture of two bivariate Gaussian components. Thus, much of the popularity of mixture models in recent years is due to the existence of efficient algorithms for estimating the set of parameters for the model. Specifically, Expectation Maximization (EM) algorithm $(113,114)$ obtains maximum-likelihood parameters in the presence of missing data by a maximization mixture log-likelihood expression:

$$
\mathcal{L}(\theta \mid X)=\sum_{i=1}^{N} \log \left(\sum_{j=1}^{k} \alpha_{j} g\left(x_{i} \mid \mu_{j}, \Sigma_{j}\right)\right)=\sum_{i=1}^{N} \log \left(p\left(x_{i} \mid \theta\right)\right)
$$

where $\theta=\left\{\theta_{i}\right\}$, for $\mathrm{i}=1, \ldots, \mathrm{k}$ and $\theta_{i}=\left\{\alpha_{\mathrm{i}}, \mu_{\mathrm{i}}, \Sigma_{\mathrm{i}}\right\}$, that is maximizing (3.20)

$$
\theta^{*}=\underset{\theta}{\operatorname{argmax}} \mathcal{L}(\theta \mid X)
$$

The application of the EM algorithm assumes that the number of $k$ components is fixed. However, in practice, it is frequently the case that $k$ is unknown. To cope with this issue, two 
standard model selection techniques have been extensively used in order to estimate the model dimensionality: penalized likelihood methods and cross-validation (CV).

Bayesian and penalized likelihood methods such as Akaike Information Criterion, AIC (115) and Bayesian Information Criterion, BIC (116) provide general frameworks for estimating the number of components by seeking a balance between model complexity and accuracy including a penalty term for the number of parameters:

$$
\begin{gathered}
B I C=-2 \log L+k \log n \\
\text { AIC }=2 k-2 \log L
\end{gathered}
$$

where $L, k$ and $n$ are log-likelihood, number of components and number of parameters in the mixture model, respectively.

Cross-validation is an intuitive iterative model selection in which a subset of the data is used to estimate the model and the rest of the data is used to evaluate its goodness. This is repeated for a number of times and in each repetition the data are randomly splitted. When the model complexity is unnecessarily high or too low, the model overfits the training data and fails to represent the test data $(117,118)$. A popular approach to perform cross-validation model selection is the one proposed by Smyth (117). Briefly, the data are repeatedly partitioned in two unequal sets, one of which is used to build the model and the other is used to evaluate the statistic of interest. The test set is shifted every time. In this work, a slight variation of the cross-validation approach is introduced, using an average of the BIC instead of using the log-likelihood as a fitting measure.

Despite all of these considerations, complex data sets are not easily modeled as a weighted sum of independent Gaussian or Student's $t$ distributions, as data are usually not normally distributed. If this is the case, a data transformation as the Box-Cox can be used in order to normalize it. The transformation proposed by Box and Cox is a parametric power transformation technique that permits to reduce abnormalities in the data such as non-additivity, non-normality and heteroscedasty (119). The Box-Cox transformation of an observation is defined as follows: 


$$
\mathrm{y}^{(\lambda)}=\left\{\begin{array}{cc}
\frac{\mathrm{y}^{\lambda}-1}{\lambda}, & \lambda \neq 0 \\
\log \mathrm{y}, & \lambda=0
\end{array}\right.
$$

where $\lambda$ describes the transformation parameter.

In order to combine the multiparametric information derived from the MRI acquisition, different regions in the brain were defined using its microstructural and biochemical characteristics. For this purpose we tested two finite mixture models (FMM) using the Expectation Maximization (EM) algorithm $(113,114)$. FMM allows the overlapping of clusters (partial volume effect), handling uncertainty about cluster membership. The second FMM model, the finite Student's-t mixture model (120) alleviates the sensitivity of these methods to the presence of outliers, which results particularly efficient in brain imaging classification (121).

We first combined penalized likelihood methods, such as Akaike Information Criterion (AIC) (115) and Bayesian Information Criterion (BIC) (122), and cross-validation methods to estimate the dimensions and shape of the probability functions of the FMM. Additionally, the need of previous preprocessing of the data by the Cox-Box transformation is evaluated. In order to select the multivariate model which better fits the data in a reasonable time, $10 \%$ of the data was used. For avoiding the convergence to local maximum, the EM algorithm is repeated 100 times with different initial parameters. Afterwards, and using the entire group-average parametric maps, the mixture of multivariate distributions and transformation selection were estimated simultaneously using the approach proposed by Lo and colleagues (81) and implemented in the R library "flowClust". Finally, probability maps of belonging to the different components in the model were created using the posterior probability.

To create a subject-specific version of the probability maps, tissue class templates in the segmentation approach implemented in SPM were replaced by the probability maps. The multimodal ROls were defined using the largest posterior probability of belonging to a specific component in the subject-specific probability maps. 


\subsubsection{Feature extraction, ranking and support vector machine learning classification}

We have used machine learning approaches above described to identify the metrics that accurately classify individuals according to the experimental conditions. Because the features might be non-uniform within the $\mathrm{mROI}$ and in order to entirely describe the MRI metrics distribution, seven statistics were obtained: mean, standard deviation, median, first and third quartile, skewness, and kurtosis. Therefore, 28 features (seven measurements and four MRI modalities) per subject and $\mathrm{mROI}$ were obtained. Note that this technique is also known as Texture Features widely used in tumor differenciation $(123,124)$. The utility of this multimodal descriptions or signatures to identify and characterize pathological conditions or disease stages, that we have called MSDs, will be explored in the following sections and chapters. Only for displaying purposes, a Principal component analysis (PCA) is assessed (125) to represent the high-dimensional data in a lower-dimensional space. PCA is an unsupervised linear feature extraction technique which transforms highdimensional (possibly correlated) data into smaller set of uncorrelated and therefore orthonormal variables (called principal components). From the complete d dimensional space, PCA projects the data to the $k$ dimensional space $(k<d)$ by maximizing the variance in the data. To visualize the data in a two-dimensional we saved the two components explaining higher variance.

The performance of the classification can be easily degraded by the presence of redundant features and therefore the selection of variables that have a discriminatory power is highly recommended. The classification task was assessed following two different strategies: Feature selection and classification as independent steps in our analysis or including both steps in a nested loop. We performed feature selection by using the Random Forest (RF) algorithm (126) and the error rate determined the importance of a given feature. In order to reduce the effect of randomly choosing the variables, the algorithm was run 100 times with 1000 trees and 14 variables randomly sampled as candidates at each split. The classification model was performed by Support Vector Machine (SVM) with linear kernel. For more detailed information refer to section 3.2.4.

Validation and optimization of the classification algorithm was performed by a crossvalidation strategy. For this, the entire data set is partitioned into complementary subsets: the 
training and the testing set. The classification model is trained using the training set and the validation is performed on the test set. Specifically, we used $k$-fold cross validation with $k=5$ (for Naïve/Exposed classification) or $k=3$ (for the Wistar/msP Exposed/Abstinence, Exposed/Naltrexone and Saline/Naltrexone classification). The entire subset was split into $k$ complementary subsets; $k-1$ of them was used to train the SVM classifier and the remaining one to test the model (testing set). The testing set was shifted every run. In addition, the splitting of the data was repeated 100 times. In order to avoid interdependence between training and testing set in those analysis of longitudinal experimental conditions (msP Rats/Wistar, Naïve/Exposed, Exposed/Abstinence and Exposed/Naltrexone), testing and trainings set were selected subject-wise meaning each set contains both samples. As well, balanced group distribution was forced in the training of the model to discriminate msPs and Wistar rats by random selection of 10 out of $18 \mathrm{msPs}$ rats. Once variable importance has been estimated, models differing in the number of used variables are implemented. For this purpose, the model is fed with an increasing number of features on each iteration and evaluated in terms of accuracy, sensitivity, specificity and AUCROC. The optimal classifier dimensionality was selected by maximizing the classification accuracy. In order to compare the performance of different models (univariate vs. multivariate with feature selection), multivariate models without previous feature selection is considered as the reference. The complete analytical workflow is summarized in Fig. 3.6. 


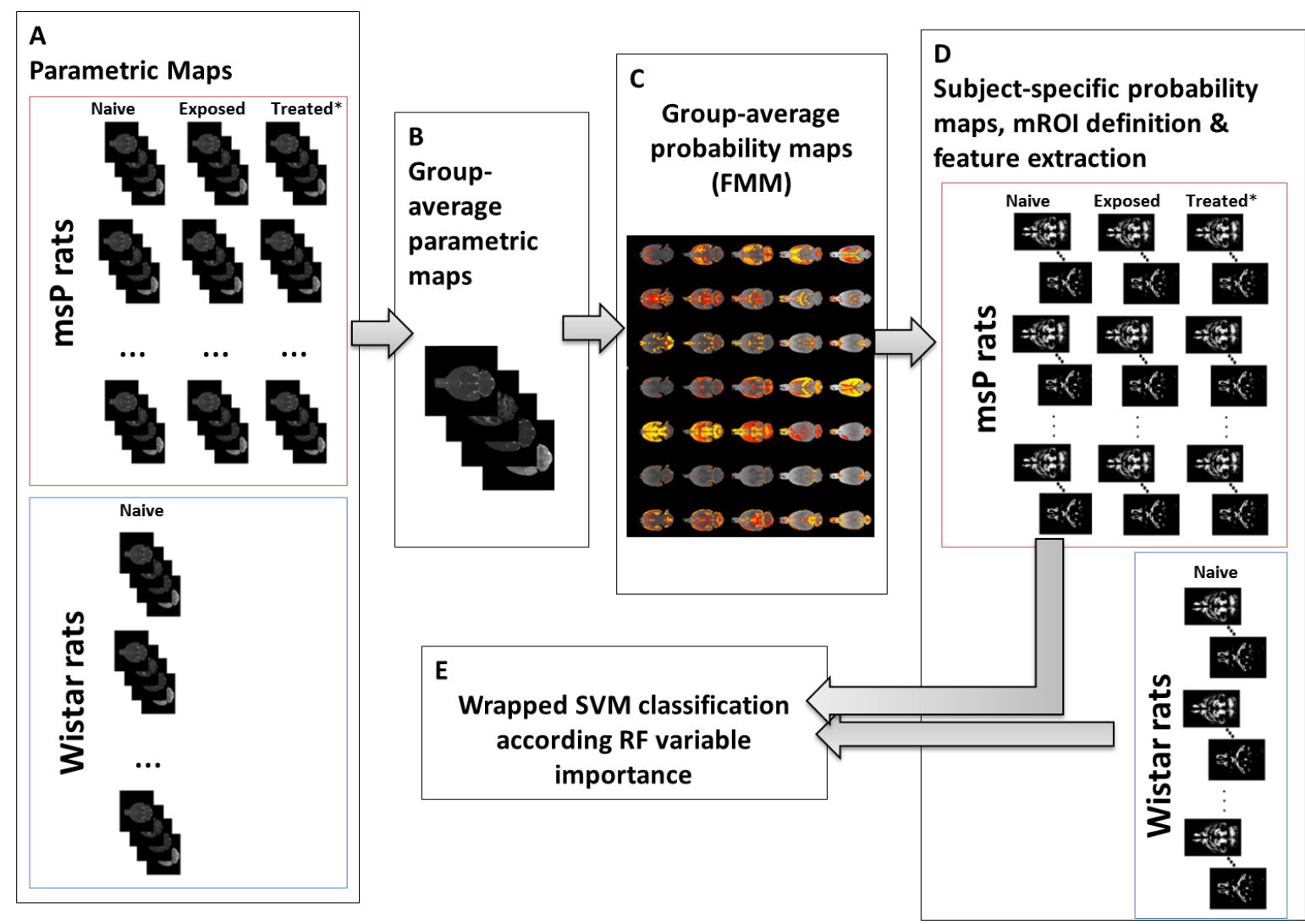

Figure 3.6 Framework of the proposed procedure. A. The parametric maps of each msP subject at different time points and Wistar rats are calculated. B. The group average parametric maps are created. C. A mixture of probability functions is created to model the group average parametric map and probability maps are created using the posterior probability of belonging to each component. D Subject-specific probability maps are projected and multimodal regions of interest (mROIs) are defined using the largest posterior probability delimitating spatial multimodal clusters. E. Statistical parameters of the distribution of the parametric values within each cluster are used as feature to discriminate different experimental stages based on the classification model proposed in the Section 3.2.4. [Basel. -Baseline, Post.Alc. Post alcohol exposition, Post.Treat. Post Naltrexone/Saline treatment]. 


\subsection{A mesoscopic multimodal machine learning approach from alcohol initiation to treatment in $\mathrm{msP}$ rats}

Here we explore the simultaneous combination of multiple parameters extracted from different MRI modalities such as mean diffusivity (MD), fractional anisotropy, T1 and T2 relaxometry times and voxel-based morphometry-derived maps to detect regional different multimodal MRI signature at different disease stages of a longitudinal experiment with in the Marchigian-Sardinian (msP) alcoholpreferring rats (127) that involves from the naïve state to the treatment with the opioid antagonist naltrexone. Besides being able to identify subjects at different experimental or clinical conditions, identifying mechanisms characterizing such conditions and biologically interpret them is fundamental for the advance in the understanding of the disorder. Specifically, multimodal prediction models are trained to identify those areas that have changed its structural properties after a month of heavy drinking by training voxel-wise classifiers allowing to make predictions in high spatial resolution. Having demonstrated the advantages of our method in terms of enhanced sensitivity to detect brain alterations, brain tissue progression of Naltrexone treated animals during the abstinence are assessed. In addition, relation between subject- and voxel-wise predictions and were related to behavioral aspects.

\subsubsection{Feature extraction, ranking and support vector machine learning classification}

Multivariate models were used to identify brain regions that discriminate multimodal MRI data set collected in two longitudinal conditions of our experiment (naïve and post EthoH exposure). Detailed introduction of machine learning approaches can be found at Section 3.2. Voxel-wise SVM classifiers were trained and tested as follows. After computing and processing parametric maps from multimodal MRI acquisition, for each voxel in the stereotaxic coordinates, multimodal voxelwise MRI fingerprint is defined as the multidimensional vector of MRI features describing its microstructural (FA and MD), macrostructural (GMv and WMv) and biochemical (T1 and T2 relaxation times) properties. Adopting the definition of MSD above introduced (section 3.3) to voxel resolutions, we introduce the concept of local MSD or simply IMSD. The dimension or cardinality $\mathrm{N}$ of the IMSD is 6 in our dataset (six MRI features). The importance of each MRI modality or 
dimension in the voxel-wise fingerprint in the ability of discriminating naïve and alcohols exposed animals (first and second multimodal MRI session) is determined using a 1000 trees RF algorithm. Afterwards, and also for each voxel, $\mathrm{N}$ classification models were computed in a forwardly step-wise fashion according the RF output. First SVM model is fed with the most important modality, the second one with the two most important and so on. Models are trained and validated by a k-fold cross-validation strategy. The misclassification hyperparameter $\mathrm{C}$ is set to 1 . For the validation of the classifiers, an internal stratified 5-fold cross validation (repeated 10 times in order to reduce the variance) is performed for each model dimensionality. In order to avoid dependencies of training and validation data, samples in the training/testing set included Naïve and Exposed IMSD of an individual. Finally, model showing a higher performance in terms of accuracy is selected for each voxel as the multimodal model that better discriminates both conditions (although it may be actually fed with a single modality). Under the null hypothesis that the linear SVM classifier cannot discriminate both conditions, the accuracy was assumed to follow a binomial distribution $B i(N, p)$ (where $\mathrm{N}=36$ samples and $\mathrm{p}=0.5$ represents the probability of each group). Local accuracies were transformed to probability of higher accuracy than no information rate and subsequently transformed to $Z$ scores. $Z$ values was mapped in the standard space and thresholded at the level of $4.75\left(p<10^{-6}\right)$. Significant clusters were obtained using the GRF implemented in FSL toolbox. Note that permutation tests would be computationally high demanding (1000 label permutation $\times 60000$ voxels) given that Null model has to be determined by label-permutation for each of the voxels. Binomial distribution is well-approximated to accuracy distributions $(128,129)$. To test the procedure, voxel-wise SVM models that show a significant accuracy are used to estimate the local progression of the alcohol abstinence (130).

As well, conventional mass-variate statistical analyses of the multimodal parametric maps were conducted. FA, MD, T1 and T2 relaxometry maps and GM and WM modulated segments of the animals in the Naïve and Post-exposure instant were compared using a paired t test implemented in SPM to compare with multivariate results. In addition to evaluate the ongoing brain maturation, effect of alcohol consumption was compared to one month of isolation in the home-cage by twosample t-test of the differences between parametric maps. Statistical maps were voxel-wise corrected at $p_{F W E}<0.05$. 
Additionally, to confirm the effect of the effect of alcohol consumption against the ongoing brain changes, ROI wise analysis was performed in well-known anatomical areas affected by heavy alcohol consumption (nucleus accumbens, caudate-putamen, cingulate cortex, hippocampus, primary somatosensory cortex and insular cortex). As MRI parameters of the same subject cannot be considered independent to each other, ROI is defined as within-subject. Specifically, mixed ANOVA with Group (Alcohol exposed or Control) as between-subject factor and ROI as withinsubject factor was performed. To test the effect of abstinence, two-way repeated measurements ANOVA was conducted. Conditions (Naïve, EtOH consumption and abstinence) and ROls were defined as within-factors. If required, post-hoc tests were adjusted by Bonferroni corrections.

\section{$\underline{\text { Treatment effect }}$}

Having optimized the voxel-based SVM models identifying the hyperparameters and dimensionality than produces the best performance in terms of accuracy in the classification, data sets derived from animals in abstinence with and without treatment were used for testing. Leveraging models trained, obtained decision values in the rank $[-\infty,+\infty]$ were scaled to posterior probability using the sigmoid function (98) and the treatment effect (Naltrexone or Saline injected) compared using a two sample test GLM implemented in SPM. We define the exposed state prediction as the probability of being classified as exposed animal where values of 0 mean absolutely classified as Naïve and values of 1 represent absolutely classified as exposed. Note that exposed state can be viewed as a one-dimensional projection of the multidimensional multimodal fingerprint. Significance threshold was set to $p<0.001$ for the primary threshold and cluster corrected for multiple comparison at the level of 0.05 .

\section{Relationship Support vector machine (SVM) decision values and alcohol preference}

Similarly to the treatment comparison we sought for the relation of IMSD's and the behavioral dimension, the relationship between sigmoid transformation of the decision values and preference ratio was investigated by Linear Regression implemented in SPM (129). We explored the relationship between alcohol preference and decision values at Naïve and exposed state. We also investigate the dependence of multimodal tissue progression during the abstinence and alcohol preference. Significance threshold was set to $p<0.001$ for the primary threshold and cluster 
corrected for multiple comparison at the level of 0.05 . 


\section{Results}

\subsection{Preliminary results}

A pilot study with substantial analytical differences from the main framework proposed was performed. Results were presented in the 35th Annual International Conference of the IEEE Engineering in Medicine and Biology Society (EMBC'13) celebrated in Kyoto (131).

Cosa, A., Canals, S., Valles-Lluch, A., \& Moratal, D. (2013). Unsupervised segmentation of brain regions with similar microstructural properties: application to alcoholism. Conf Proc IEEE Eng Med Biol Soc, 2013, 1053-1056.

In this work, a novel brain MRI segmentation approach was used to evaluate microstructural differences between groups. Going further from the traditional segmentation of brain tissues (white matter -WM-, gray matter -GM- and cerebrospinal fluid -CSF- or a mixture of them), a new way to classify brain areas was proposed using their microstructural MR properties. Eight rats were studied using the proposed methodology identifying regions which present microstructural differences as a consequence on one month of hard alcohol consumption. Differences in relaxation times of the tissues were found in different brain regions $(p<0.05)$. Furthermore, these changes allowed the automatic classification of the animals based on their drinking history (hit rate of $93.75 \%$ of the cases).

Detailed explanation of methods and results can be found in the Appendix I.

\subsection{Multimodal magnetic resonance imaging as a tool for identifying the different stages of alcohol addiction}

During the 1 month period allocated for drinking in the two bottle free-choice paradigm msP animals consumed high levels of alcohol $(6.8 \pm 1.4 \mathrm{~g} / \mathrm{Kg} /$ day, mean $\pm \mathrm{SD})$. All animals completed the three scheduled imaging sessions and data quality was comparable across sessions and animals (data shown in Chapter 3). The results of the multimodal imaging study are presented as follows: (1) selection of the number of components and probabilistic functions of the FMM, (2) definition of 
different mROIs and their anatomical distribution, and (3) performance of the classifiers to discriminate between different stages in the alcohol use disorder model.

\subsubsection{Definition of the Finite Mixture Model}

A small sample of data (10\% of the total number of observations) was used to estimate the optimal number of components for the mixture models (see Methods). The results of the BIC analysis for mixtures from 1 to 30 distribution functions are shown in Figure 4.1.A. Both Gaussian and Student't $t$ distribution models were tested, with or without Box-Cox transformation. An optimal statistical description of the data is obtained from 7 components onwards, as indicated by the maximal BIC values, for both models. Finite mixture of Student's t distributions shows higher fitting than mixture of Gaussians distributions. This finding is consistent with previous reports stating that the use of longer-tailed distribution's $t$ is more robust against the presence of outliers within the data (120). It is also important to highlight that the fitting is improved when the data has been previously transformed by the Box-Cox procedure (81) most likely due to the skewed distribution of the DTI data.

The probability maps of belonging to every component of the seven selected above are provided in Figure 4.1.B. The obtained mROls show remarkably similar neuroanatomical demarcations with both methods, the mixture of Gaussian (A) and the Student's t distribution (B), illustrating the robustness of the approach. 
A
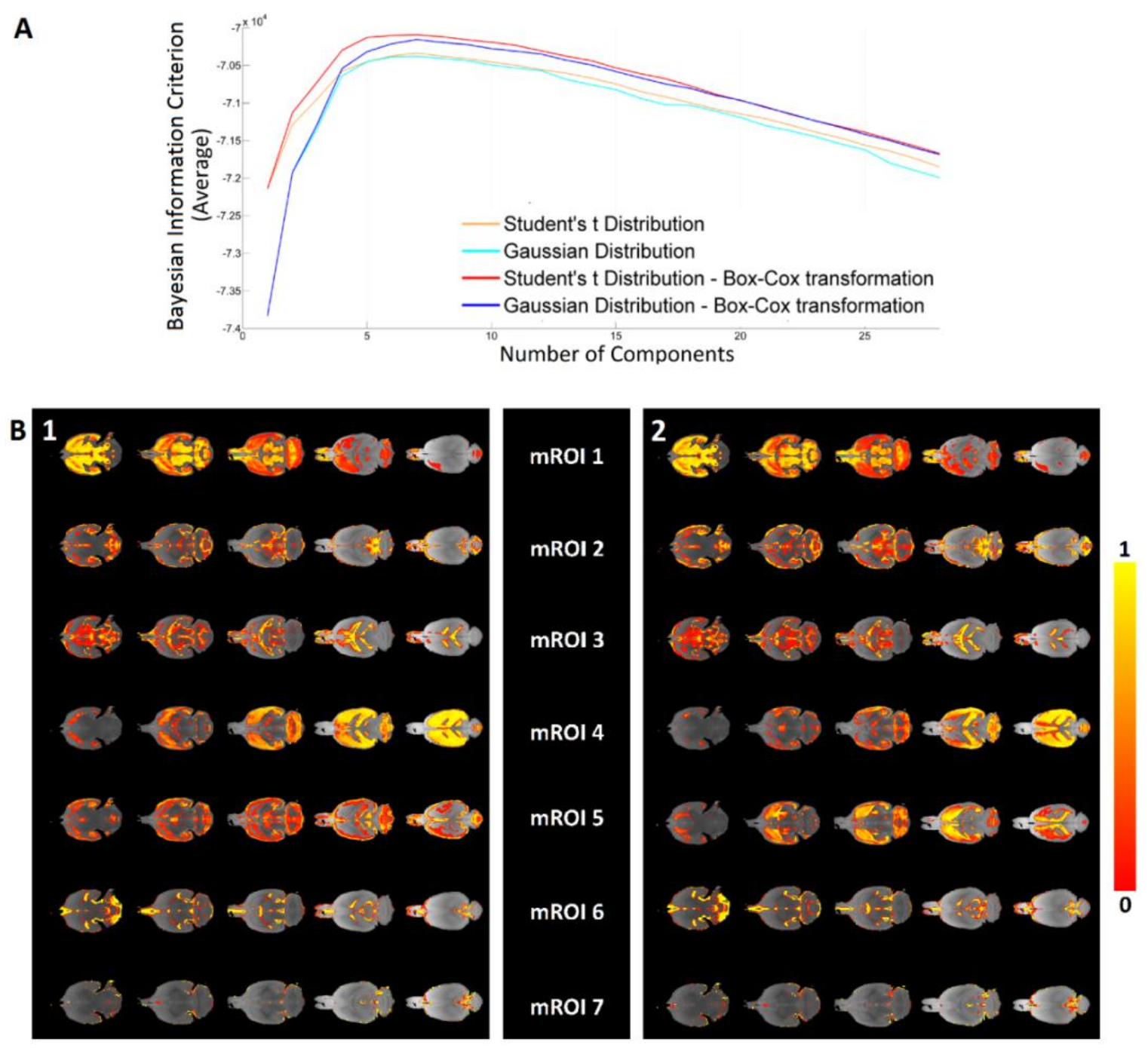

Figure 4.1 Model definition. (A) Average BIC values with cross-validation procedure ( $k=10$ folds) from 1 to 30 components for both Gaussian and Student's t distributions with or without Box-Cox transformations (see legend). (B) Five slices of posterior probability maps (in columns) for belonging to 7 different components (rows). Gaussian (B.1) and Student's t (B.2) components are showed for comparison. Note the bilateral anatomical symmetry of the components (mROIs) and the remarkable similarity of the results obtained with both models. Reproduced from (77).

As can be seen in Fig. 4.1B, the variety and complexity of mROI's largely surpass any classical parcelation of anatomical territories or segmentation of brain tissues. For instance, individual mROIs may expand the boundaries of gray matter, white matter, and cerebrospinal fluid, or combine cortical and subcortical territories, to mention some examples. All mROI's, however, show a welldefined midline symmetry, consistent with the bilateral (left-right) symmetry of the central nervous 
system, particularly patent in the rodent brain. In virtue of this results we conclude that to reach a good fitting of the data the model should accomplish the following points: (1) the dimension of the model should be of 7 components whether Gaussian or Student's t distributions are used; (2) the previous Box-Cox transformation is mandatory in order to achieve a more normally distributed data, particularly for data derived from the DTI such as FA and MD; (3) robust and anatomically meaningful mROls are defined by the 7 components of the model. In the next steps of the analyses were performed defining 7 multimodal ROls based in the aforementioned characteristics (Fig. 4.2 and Fig. 4.3).
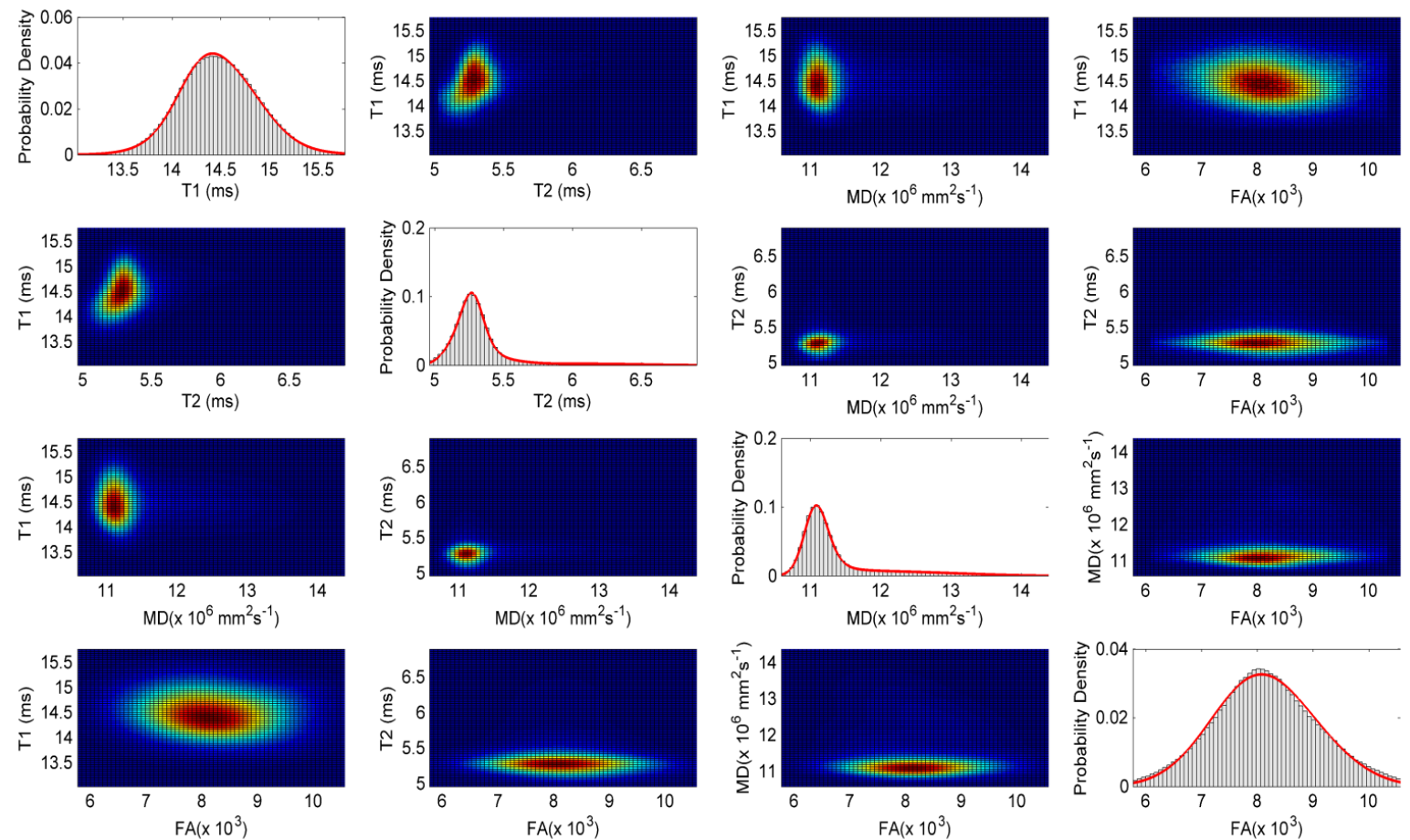

Figure 4.2 Fitting of the 4D data by mixture of seven Student's $t$ distribution. In the upper diagonal, 2-by-2 modality joint distribution of the actual data and in the lower diagonal fitted distribution. Diagonal shows the histogram of each modality (bars) and obtained fitting (red line). 


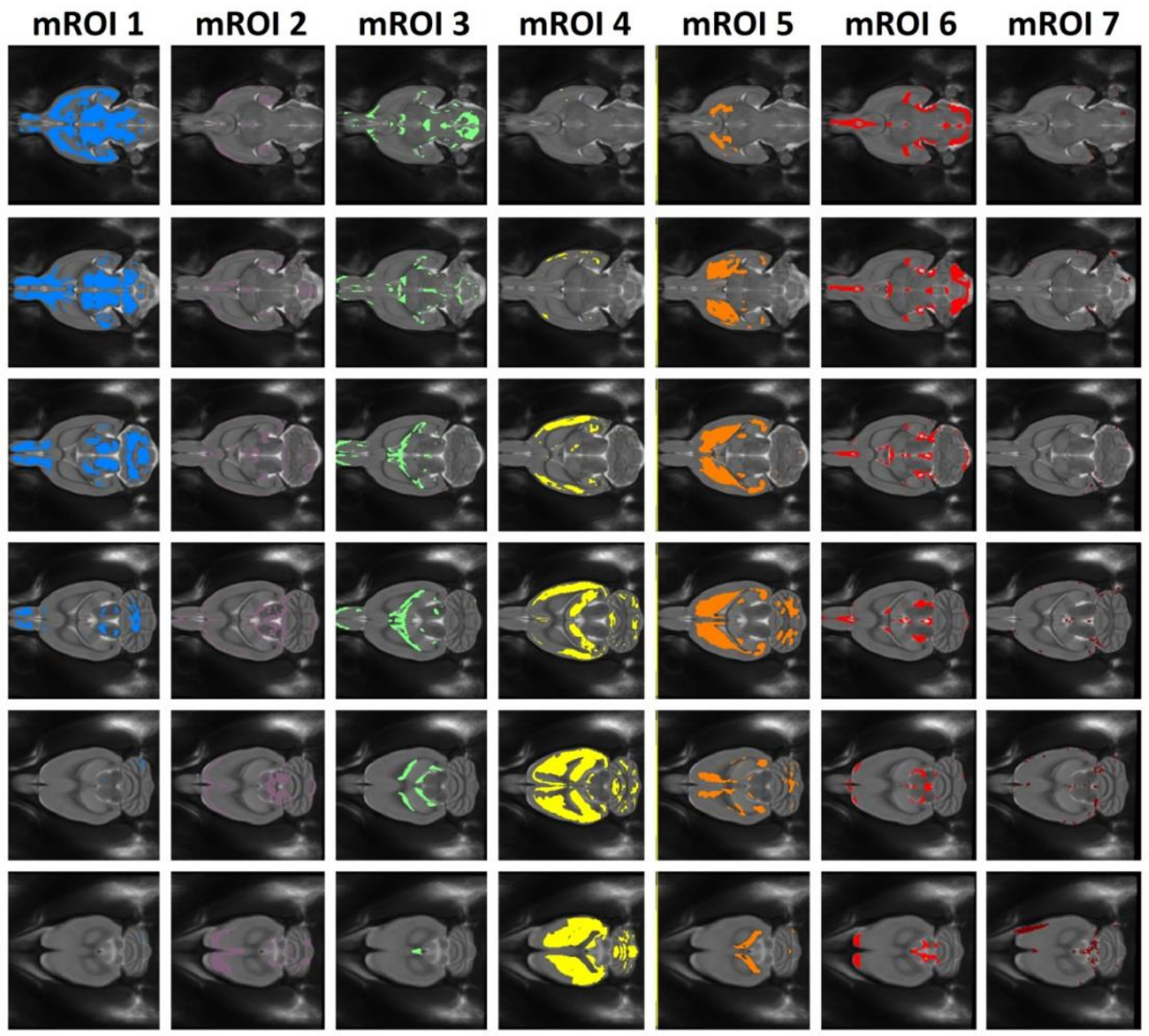

Figure $4.3 \mathrm{mROI}$ definition. Six slices (in rows) of the thresholded probability maps defining seven mROIs (columns). Note the bilateral anatomical symmetry of the components (mROIs) and the remarkable similarity of the results obtained with both models. Reproduced from (77).

\subsubsection{Multivariate analysis of MSDs}

The seven mROIS were used to parcellate the brain and obtain high order statistics to fully describe MRI parameters forming the so-called MSDs characterizing different experimental stages. As an explorative analysis we conduct a PCA to identify a reduced set of uncorrelated variables. By simple visual inspection, we observed that different experimental stages can be easily discriminated when multiple MRI modalities are combined together (Figure 4.4A). Unimodal set of features hardly 
separate experimental states in the reduced space (Figure 4.4B). Only MD-derived features isolate Naïve experimental state from the rest. Moreover, 4-class (excluding Wistar data sets) RF variable importances of MD-derived measures are representing the $37 \%$ of the contribution against the $32 \%$ of T2-derived measures. As expected from figure 4.4A, T1 and FA features contribute to a minor extent in the condition discrimination ( $15 \%$ and $17 \%$ respectively).

A

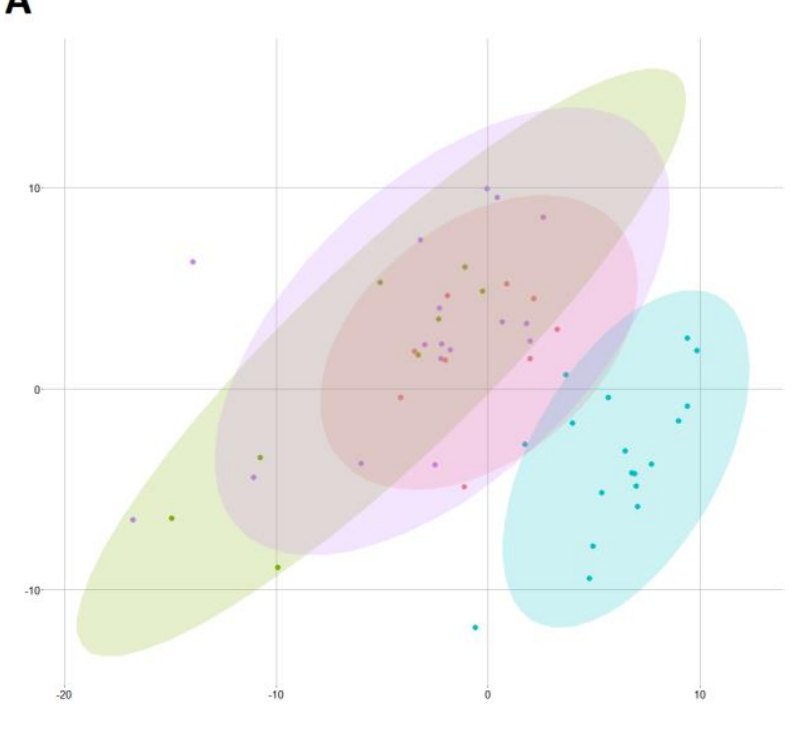

C.1 Modality

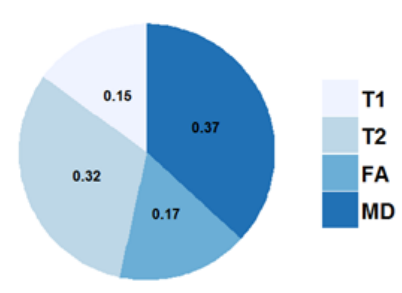

B.1

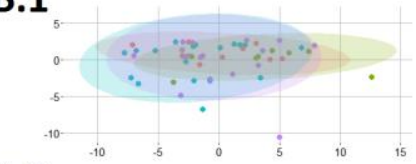

B.2

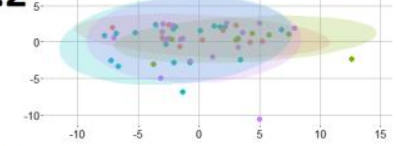

B. 3

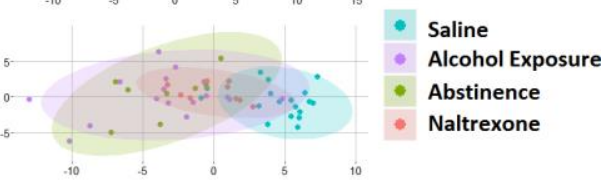

B. 4

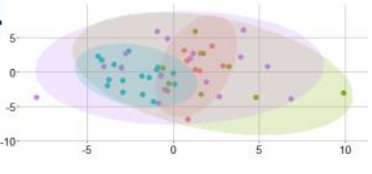

C.2 mROIS

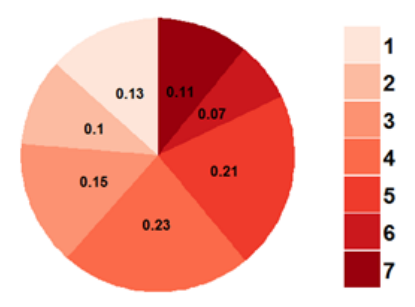

C.3 Statistics

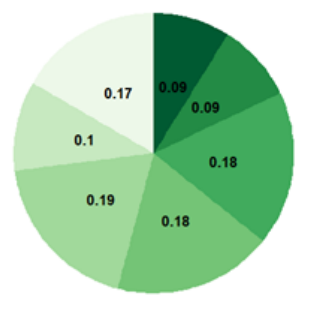

Mean

Std

1stQuart

3rdQuart

Med

Kurt

Figure 4.4 PCA decomposition and contribution of MRI-derived features to differentiate experimental conditions. PCA decomposition for 196-dimensional components derived by four modalities together $(A)$ and unimodal PCA decomposition for T1 (B.1), T2 (B.2), MD (B.3) and FA (B.4). Weight of contribution to the total variable importance grouped according modality (C.1), mROI (C.2) and Statistics (C.3). Experimental conditions are naïve, alcohol exposed, abstinence and Naltrexone treated rats. 


\section{$\underline{\text { Rat strains with different genetic predisposition to alcohol consumption }}$}

The first question is whether two different strains can be discriminated by the mROls generated in the previous analysis, namely to classify Wistar rats and msP naïve animals (with a Wistar genetic background). As previously introduced, features differ in their utility to classify subjects in their correct condition. Fig. 4.5A shows the importance of the first 40 features (out of 196) that mostly contribute to the model and, therefore, to discriminate between both rat lines. It is worth noting that 14 out of the 15 first features are measurements extracted from the MD maps. Values derived from the T1 map also contribute to the model, whereas T2 and FA measurements are less relevant for a good classification. Interestingly, statistical measures of the distribution, besides the mean, importantly contribute to a high performance. Regarding the performance indices, Fig. 4.5.B shows the mean values of sensitivity, specificity, and accuracy measured by the crossvalidation method as a function of the number of features feeding the model. The maximum performance is achieved with 150 features feeding the model. As expected, the exclusion of feature selection of the CV loop (red line in Fig. 4.5) overperforms when it is included into (blue line in Fig. 4.5) at the cost of losing model generalization. 


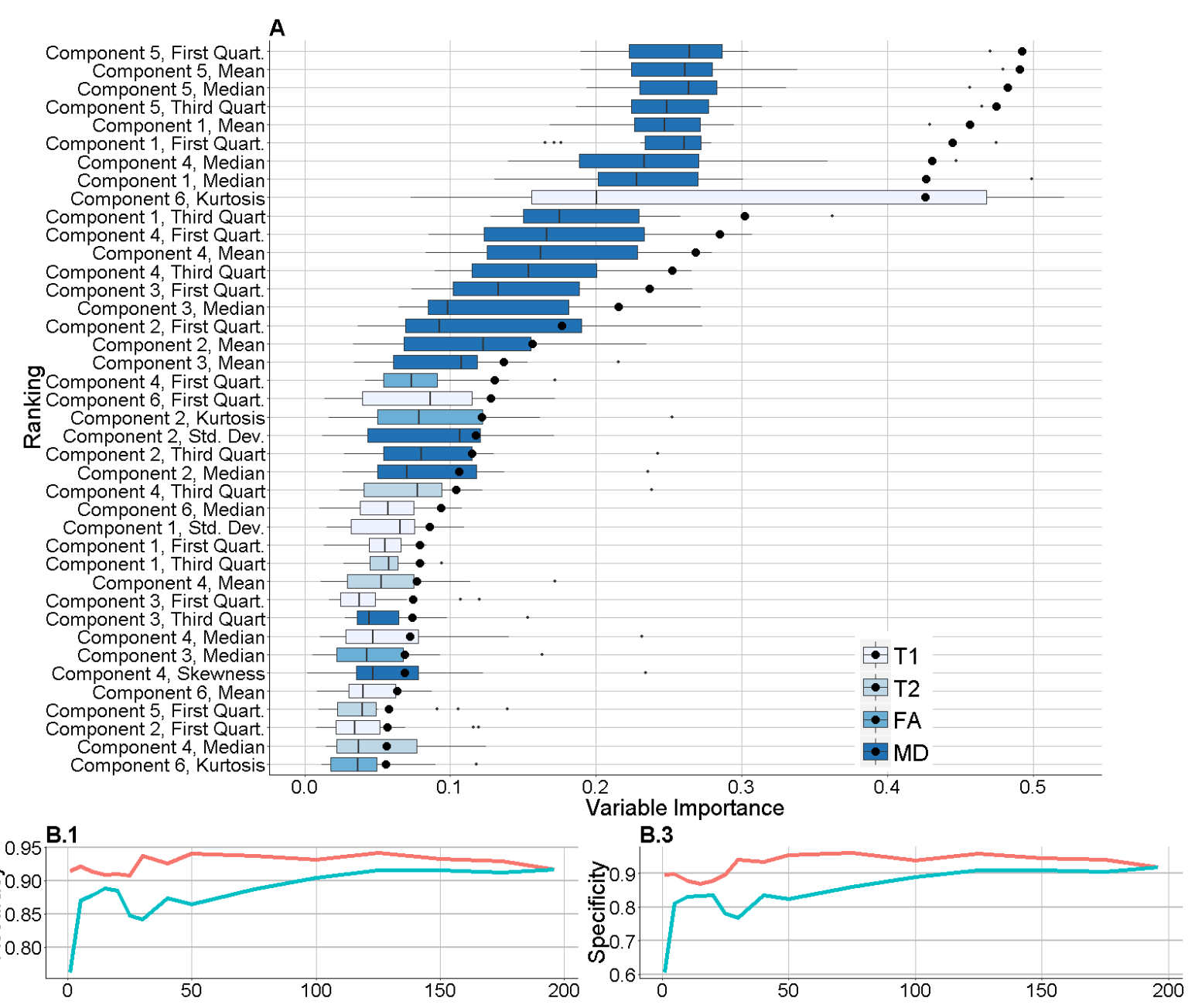

B.2

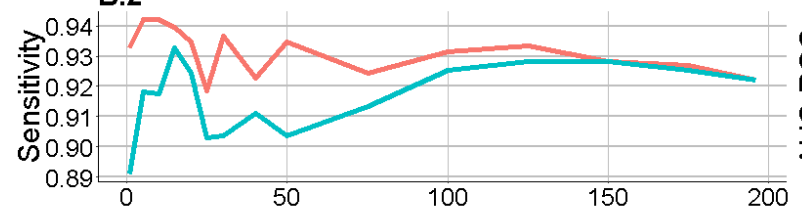

B.4

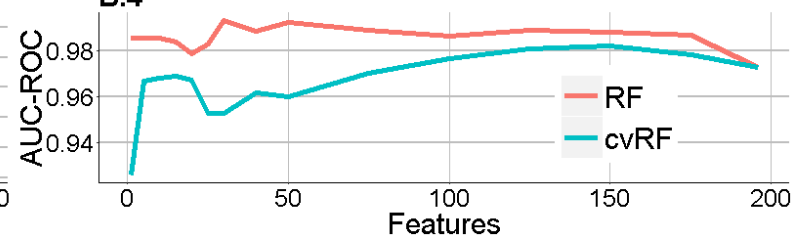

Figure 4.5 Results of the implemented model to distinguish between $\mathrm{msP}$ and Wistar subjects. (A) Ranking of features according to their importance for model performance. Accuracy (B), Sensitivity (C), specificity (C) and area under the ROC curve (AUC) of the SVM model against the number of features (D). In figure (A) boxplots represent the distribution of variable importances across folds in the CV scheme. Larger dark points show the variable importance estimated by RF with the entire data set. RF: RF out of cross-validation loop. cvRF: cross validation in the cross-validation loop.

Performances of several classification models and features contributions are depicted in Figure 4.6. Note that feature selection does not improve because the entire set of features is needed to reach maximum performance. Respect to MD classifier (BSMCA), the improvement is $1 \%$ 
in classification accuracy and $3 \%$ in sensitivity. MD unimodal and multimodal classifiers show performances above by chance (Table 4.1)

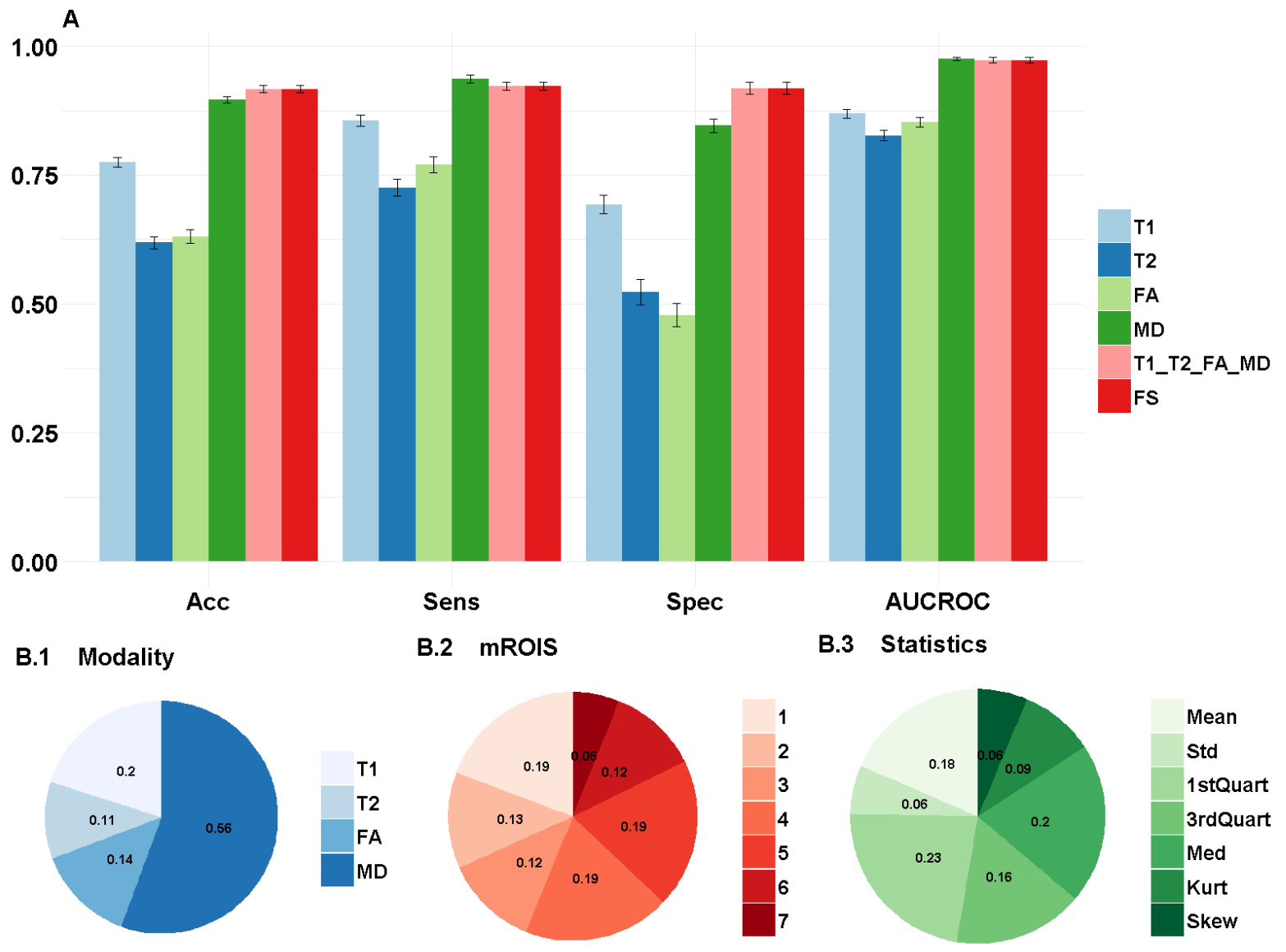

Figure 4.6 Performance of unimodal and multimodal SVM models to identify msP rats from the Wistar control. (A) Performance in terms of. Accuracy (Acc), Sensitivity (Sens), specificity (Spec) and area under the ROC curve (AUCROC) of the SVM model. Contribution of selected features grouped according modality (B.1), mROI (B.2) and Statistics (B.3). T1: Model trained with T1-derived features; T2: Model trained with T2-derived features; FA: Model trained with FA-derived features; MD: Model trained with MD-derived features; T1_T2_FA_MD: Model trained with all features without feature selection. FS: Model trained with all features with feature selection. 


\begin{tabular}{|c|c|c|c|c|c|c|c|c|}
\hline \multirow[t]{2}{*}{ Models } & \multicolumn{2}{|c|}{ Accuracy } & \multicolumn{2}{|c|}{ Sensitivity } & \multicolumn{2}{|c|}{ Specificity } & \multicolumn{2}{|c|}{ AUC-ROC } \\
\hline & $\begin{array}{c}\text { Mean } \\
(95 \% \text { C.I.) } \\
\end{array}$ & $\mathbf{p}$ & $\begin{array}{c}\text { Mean } \\
\text { (95\% C.I.) } \\
\end{array}$ & p & $\begin{array}{c}\text { Mean } \\
(95 \% \text { C.I. }) \\
\end{array}$ & p & $\begin{array}{c}\text { Mean } \\
\text { (95\% C.I.) } \\
\end{array}$ & p \\
\hline T1 & $\begin{array}{c}0.77 \\
(0.61-0.90)\end{array}$ & 0.011 & $\begin{array}{c}0.86 \\
(0.69-1.00)\end{array}$ & 0.149 & $\begin{array}{c}0.69 \\
(0.39-0.89)\end{array}$ & 0.069 & $\begin{array}{c}0.87 \\
(0.72-0.96)\end{array}$ & 0.060 \\
\hline T2 & $\begin{array}{c}0.62 \\
(0.39-0.83)\end{array}$ & 0.187 & $\begin{array}{c}0.73 \\
(0.47-0.92)\end{array}$ & 0.390 & $\begin{array}{c}0.52 \\
(0.17-0.92)\end{array}$ & 0.273 & $\begin{array}{c}0.83 \\
(0.67-0.96)\end{array}$ & 0.154 \\
\hline FA & $\begin{array}{c}0.63 \\
(0.39-0.84)\end{array}$ & 0.177 & $\begin{array}{c}0.77 \\
(0.42-1.00)\end{array}$ & 0.253 & $\begin{array}{c}0.48 \\
(0.11-0.78)\end{array}$ & 0.317 & $\begin{array}{c}0.85 \\
(0.69-1.00)\end{array}$ & 0.094 \\
\hline MD & $\begin{array}{c}0.90 \\
(0.82-0.95)\end{array}$ & 0.000 & $\begin{array}{c}0.94 \\
(0.89-1.00)\end{array}$ & 0.063 & $\begin{array}{c}0.85 \\
(0.72-0.92)\end{array}$ & 0.014 & $\begin{array}{c}0.98 \\
(0.93-1.00)\end{array}$ & 0.001 \\
\hline $\mathrm{T} 1, \mathrm{~T} 2, \mathrm{FA}, \mathrm{MD}$ & $\begin{array}{c}0.92 \\
(0.80-1.00)\end{array}$ & 0.000 & $\begin{array}{c}0.92 \\
(0.80-1.00)\end{array}$ & 0.030 & $\begin{array}{c}0.92 \\
(0.77-1.00)\end{array}$ & 0.003 & $\begin{array}{c}0.97 \\
(0.83-1.00)\end{array}$ & 0.000 \\
\hline FS & $\begin{array}{c}0.92 \\
(0.80-1.00)\end{array}$ & 0.000 & $\begin{array}{c}0.92 \\
(0.80-1.00)\end{array}$ & 0.030 & $\begin{array}{c}0.92 \\
(0.77-1.00)\end{array}$ & 0.003 & $\begin{array}{c}0.97 \\
(0.83-1.00)\end{array}$ & 0.000 \\
\hline
\end{tabular}

Table 4.1 Performance of unimodal and multimodal SVM models to identify msP rats from the Wistar control. T1: Model trained with T1-derived features; T2: Model trained with T2-derived features; FA: Model trained with FA-derived features; MD: Model trained with MD-derived features; T1_T2_FA_MD: Model trained with all features without feature selection. FS: Model trained with all features with feature selection. P-values mean significance with respect to the label-permuted null model.

A detailed comparison of performances between the implemented multivariate model and the independent univariate ones (for $\mathrm{T} 1, \mathrm{~T} 2, \mathrm{FA}$ or $\mathrm{MD}$ ), is shown in Table 4.1. The highest performance of the univariate models is provided by $M D$, as expected from the multivariate results ranking MD-related features in top position for an accurate discrimination between msP and Wistar rats (Fig. 4.5A and 4.6B.1). T1, T2 and FA classifiers show also high performances (AUCs above $80 \%$ ).

\section{Effects of alcohol consumption}

Next, having shown in a reduced space that different experimental condition can be differentiated, we attempted to discriminate in the very same set of animals, the occurrence or not of alcohol consumption. We used the mROls generated for msP animals before (positive class) alcohol exposure and the same animals after 1 month of drinking. The importance of the features to correctly discriminate between groups, as well as the performance indices is shown (Fig. 4.7). The ranked first 36 features (out of 40 ) are a combination of measurements derived from the MD and T2 maps (Fig. 4.7A). Particularly in this analysis, these 75 most important features are the ones that optimize the model according to sensitivity, specificity, and accuracy (Fig. 4.7B.1-3). However, accuracies higher than $97 \%$ are reached with only 15 features remaining practically constant thereafter. The area under ROC curve as a function of the number of features (Fig. 4.7B4) confirms 
these results.

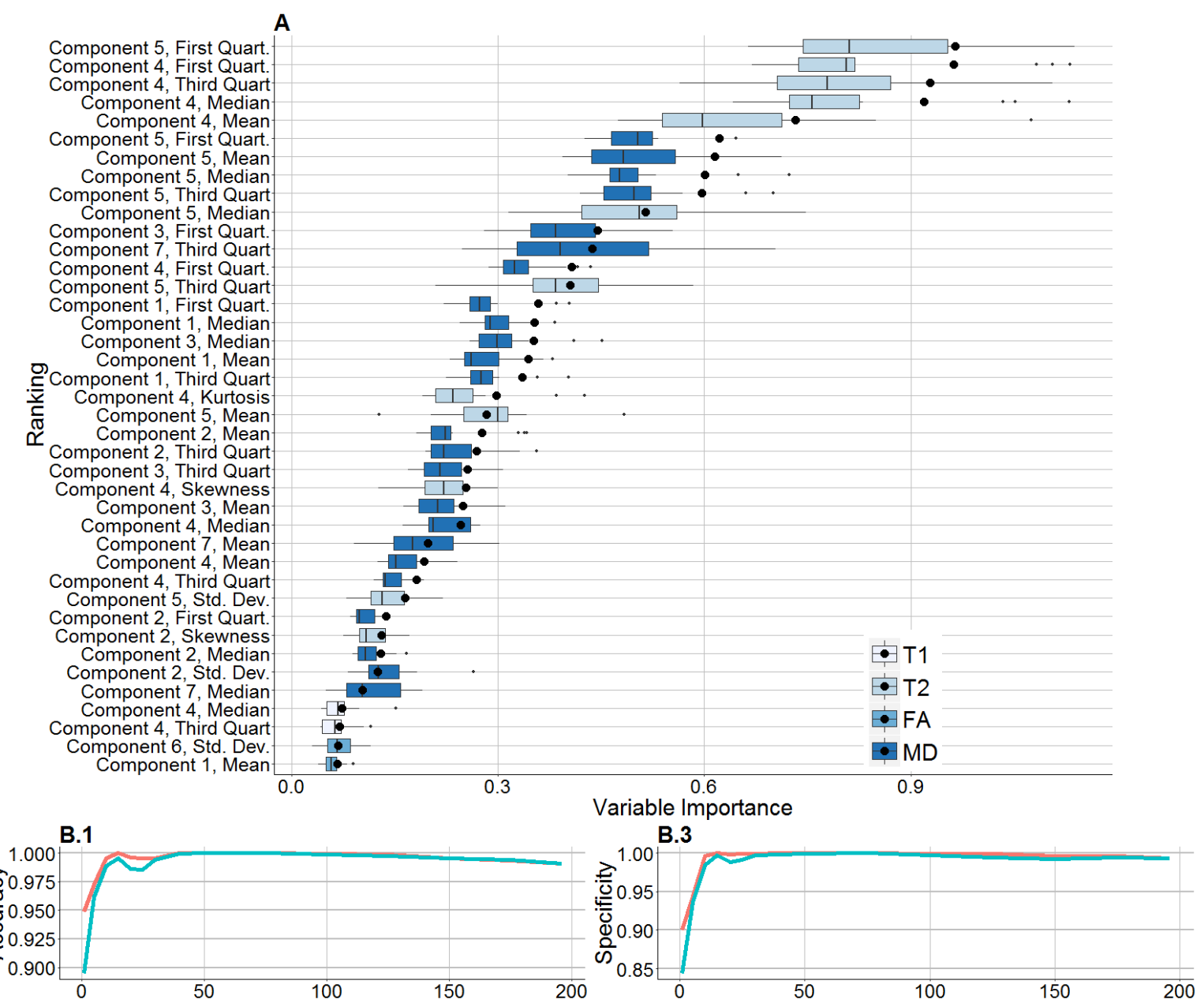

B.2

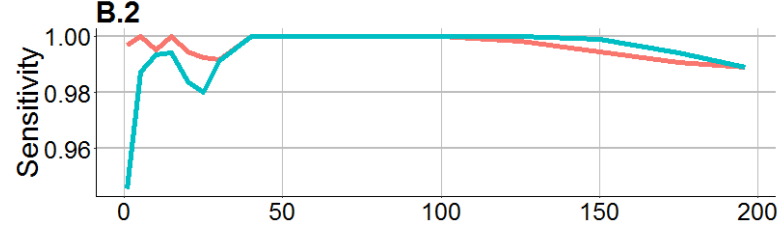

B.4

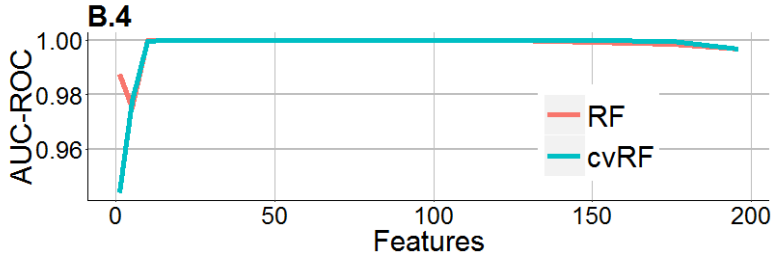

Figure 4.7 Feature selection to classify msP rats after one month of heavy alcohol consumption. (A) Ranking of features according to their importance for model performance. Accuracy (B), Sensitivity (C), specificity (C) and area under the ROC curve (AUC) of the SVM model against the number of features (D). In figure (A) boxplots represent the distribution of variable importances across folds in the CV scheme. Larger dark points show the variable importance estimated by RF with the entire data set. RF: RF out of cross-validation loop. cVRF: cross validation in the cross-validation loop.

Performing feature selection at the inner loop of the cross validation scheme does not modify the performance of the classifier demonstrating robustness against overfitting. Univariate models 
(Table 4.2 and Figure 4.8A) show a significant reduction in all performance measures with respect to multivariate. Univariate T2 and MD models (above $90 \%$ of accuracy) perform better than T1 and FA models, as expected again from the ranking of features provided by the multivariate analysis (Fig. 4.8B). All modalities alone classifies samples with accuracies higher than by chance $(p<.05)$.

\section{A}

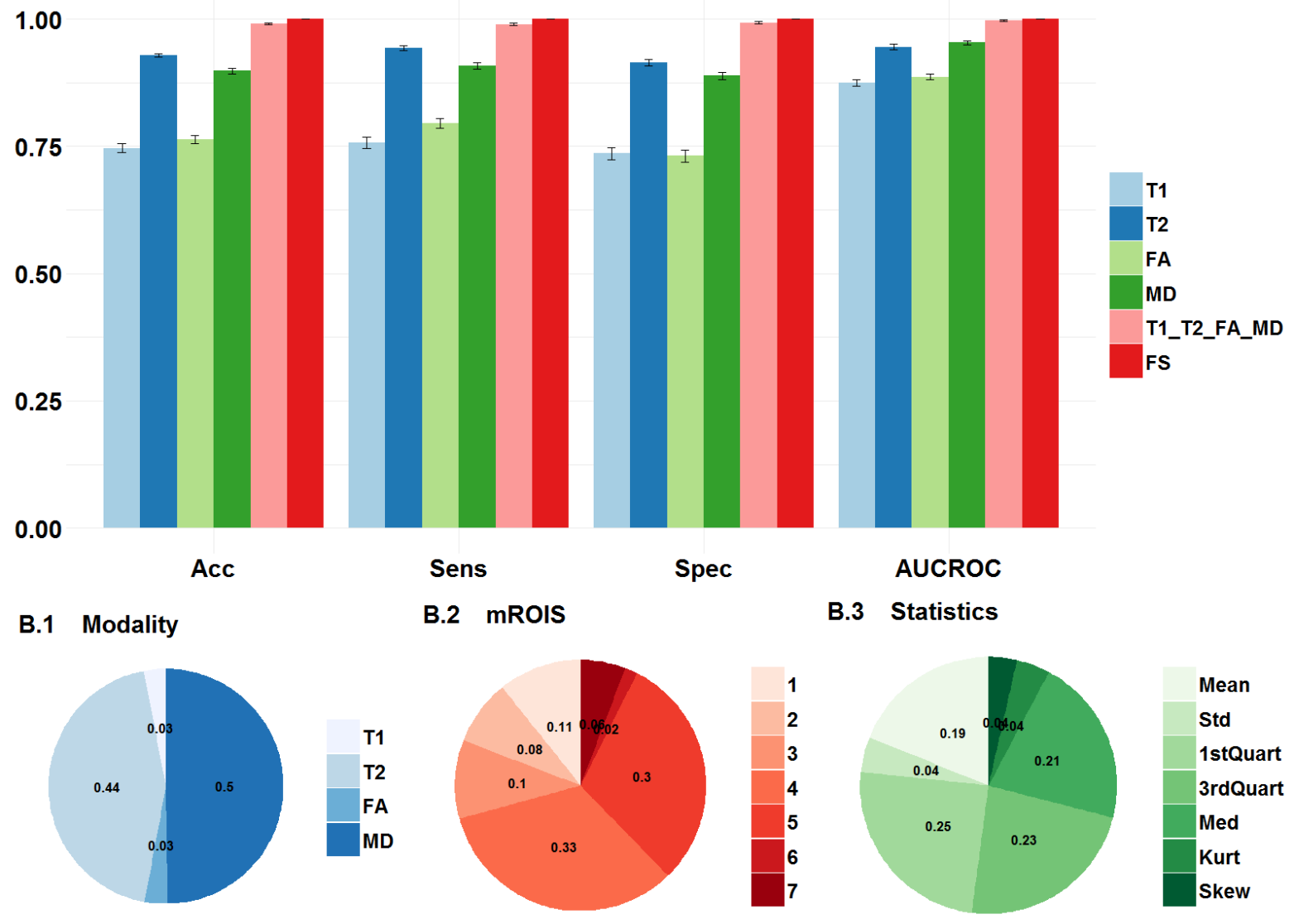

Figure 4.8 Performance of unimodal and multimodal SVM models to identify msP rats after one month of heavy alcohol consumption. (A) Performance in terms of. Accuracy (Acc), Sensitivity (Sens), specificity (Spec) and area under the ROC curve (AUCROC) of the SVM model. Contribution of selected features grouped according modality (B.1), mROI (B.2) and Statistics (B.3). T1: Model trained with T1-derived features; T2: Model trained with T2-derived features; FA: Model trained with FA-derived features; MD: Model trained with MD-derived features; T1_T2_FA_MD: Model trained with all features without feature selection. FS: Model trained with all features with feature selection.

Overall from this analysis we can conclude that using multimodal MRI information and machine learning methods the proposed framework correctly discriminate the occurrence of high alcohol drinking with an average accuracy of $100 \%$. The classification accuracy is enhanced in $7 \%$ 
respect to the best unimodal classifier (SVMT2). As well, feature selection increases the performance $(1 \%)$ reaching the perfect classification accuracy. The high performance of the univariate and multivariate models confirms the severe impact that alcohol produces in the brain tissue.

\begin{tabular}{|c|c|c|c|c|c|c|c|c|}
\hline \multirow[t]{2}{*}{ Models } & \multicolumn{2}{|c|}{ Accuracy } & \multicolumn{2}{|c|}{ Sensitivity } & \multicolumn{2}{|c|}{ Specificity } & \multicolumn{2}{|c|}{ AUC-ROC } \\
\hline & $\begin{array}{c}\text { Mean } \\
\text { (95\% C.I.) }\end{array}$ & $p$ & $\begin{array}{c}\text { Mean } \\
\text { (95\% C.I.) }\end{array}$ & p & $\begin{array}{c}\text { Mean } \\
\text { (95\% C.I.) }\end{array}$ & $p$ & $\begin{array}{c}\text { Mean } \\
\text { (95\% C.I.) }\end{array}$ & $\mathbf{p}$ \\
\hline T1 & $\begin{array}{c}0.75 \\
(0.60-0.82)\end{array}$ & 0.004 & $\begin{array}{c}0.76 \\
(0.58-0.85)\end{array}$ & 0.020 & $\begin{array}{c}0.74 \\
(0.58-0.85)\end{array}$ & 0.029 & $\begin{array}{c}0.87 \\
(0.82-0.92)\end{array}$ & 0.002 \\
\hline $\mathrm{T} 2$ & $\begin{array}{c}0.93 \\
(0.90-0.97)\end{array}$ & 0.000 & $\begin{array}{c}0.94 \\
(0.93-0.95)\end{array}$ & 0.000 & $\begin{array}{c}0.91 \\
(0.85-1.00)\end{array}$ & 0.001 & $\begin{array}{c}0.95 \\
(0.93-0.95)\end{array}$ & 0.000 \\
\hline FA & $\begin{array}{c}0.76 \\
(0.68-0.83)\end{array}$ & 0.009 & $\begin{array}{c}0.80 \\
(0.72-0.85)\end{array}$ & 0.012 & $\begin{array}{c}0.73 \\
(0.60-0.83)\end{array}$ & 0.051 & $\begin{array}{c}0.89 \\
(0.85-0.93)\end{array}$ & 0.003 \\
\hline MD & $\begin{array}{c}0.90 \\
(0.88-0.92)\end{array}$ & 0.000 & $\begin{array}{c}0.91 \\
(0.87-0.95)\end{array}$ & 0.002 & $\begin{array}{c}0.89 \\
(0.87-0.90)\end{array}$ & 0.002 & $\begin{array}{c}0.95 \\
(0.91-0.99)\end{array}$ & 0.000 \\
\hline $\mathrm{T} 1, \mathrm{~T} 2, \mathrm{FA}, \mathrm{MD}$ & $\begin{array}{c}0.99 \\
(0.97-1.00)\end{array}$ & 0.000 & $\begin{array}{c}0.99 \\
(0.93-1.00)\end{array}$ & 0.000 & $\begin{array}{c}0.99 \\
(0.95-1.00)\end{array}$ & 0.000 & $\begin{array}{c}1.00 \\
(0.96-1.00)\end{array}$ & 0.000 \\
\hline FS & $\begin{array}{c}1.00 \\
(1.00-1.00)\end{array}$ & 0.000 & $\begin{array}{c}1.00 \\
(1.00-1.00)\end{array}$ & 0.000 & $\begin{array}{c}1.00 \\
(1.00-1.00)\end{array}$ & 0.000 & $\begin{array}{c}1.00 \\
(1.00-1.00)\end{array}$ & 0.000 \\
\hline
\end{tabular}

Table 4.2 Performance of unimodal and multimodal SVM models to identify msP rats after one month of heavy alcohol consumption. T1: Model trained with T1-derived features; T2: Model trained with T2-derived features; FA: Model trained with FA-derived features; MD: Model trained with MD-derived features; T1_T2_FA_MD: Model trained with all features without feature selection. FS: Model trained with all features with feature selection. P-values mean significance with respect to the label-permuted null model.

\section{Effects of alcohol abstinence}

Having demonstrated the capacity of the framework to accurately classify alcohol consumers, the next logic step of our analysis was to investigate whether alcohol withdrawal or abstinence curse with additional alterations in brain tissue producing a distinct multimodal signature. For this analysis we used the same mROls of the alcohol exposed animals in the previous section (alcohol condition), together with the mROIs of the same animals obtained one week after forced alcohol withdrawal (abstinence condition). Abstinence condition was defined as positive class. As can be seen in Fig. 4.9, a performance of the classifier was obtained for discriminating both conditions (see below). The first 20 features provide to the model the optimal information to reach the highest performance. Feature ranking for this particular optimal classification is distributed across T1 and FA and, in lesser degree, MD and and T2-derived features. 


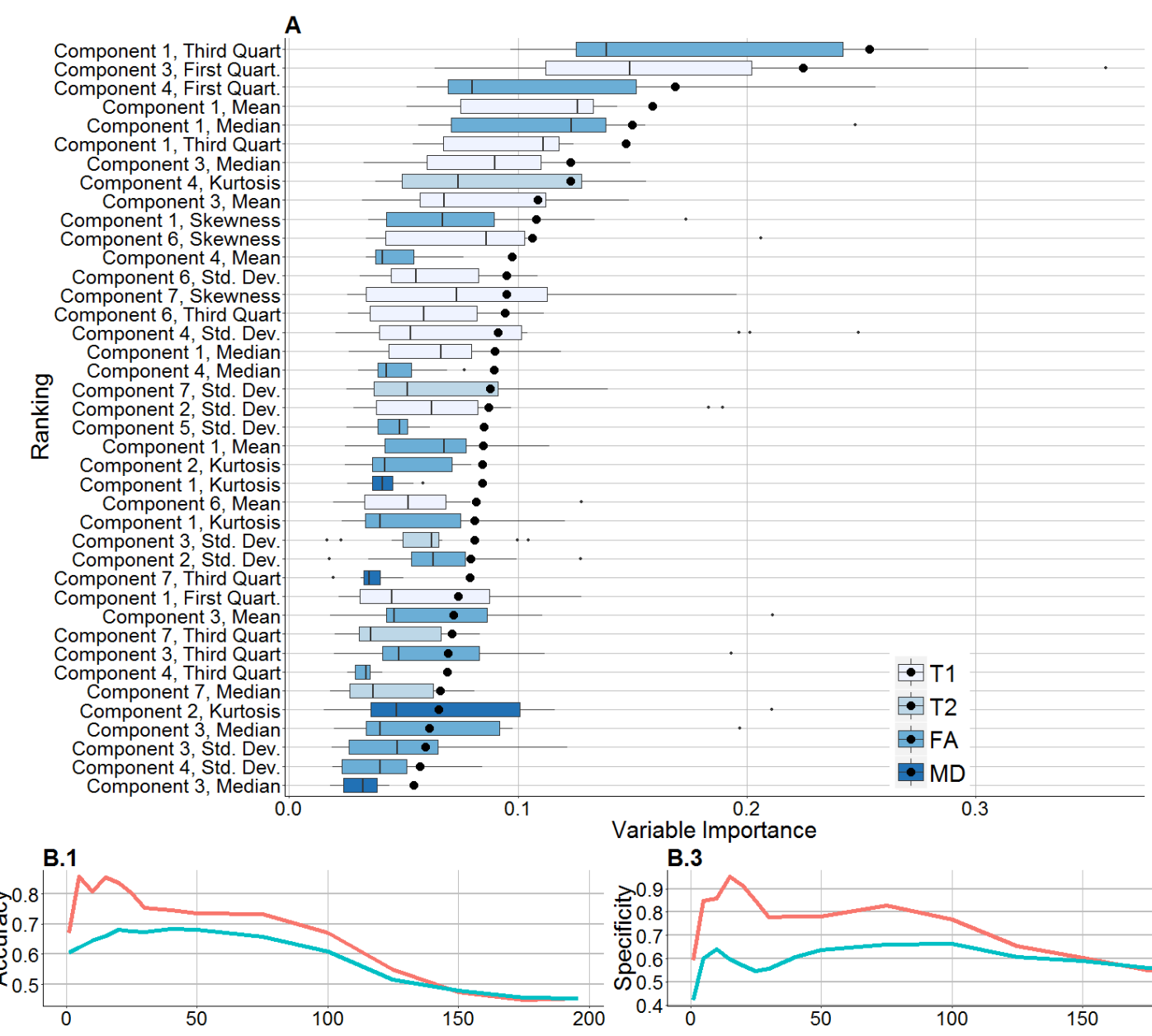

A

B.1

\section{B. 3}

B. 2

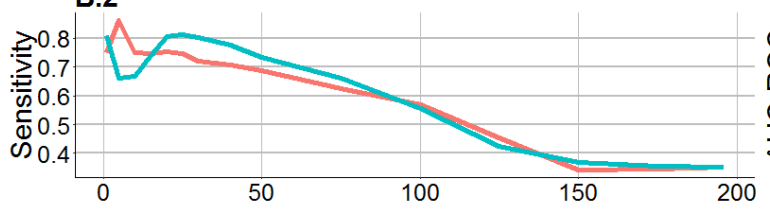

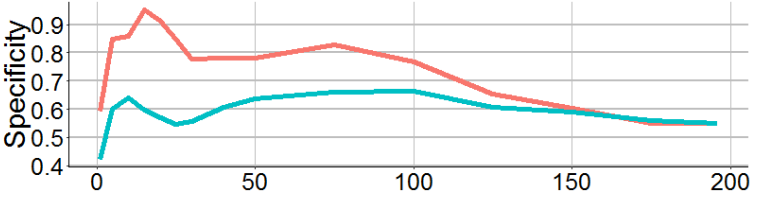

B.4

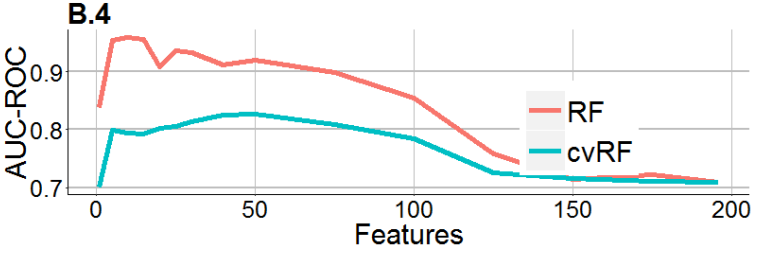

Figure 4.9 Feature selection of the implemented model to distinguish between animals in a one month continuous drinking paradigm from the same subjects after a one week of forced abstinence. (A) Ranking of features according to their importance for model performance. Accuracy (B), Sensitivity (C), specificity (C) and area under the ROC curve (AUC) of the SVM model against the number of features (D). In figure (A) boxplots represent the distribution of variable importances across folds in the CV scheme. Larger dark points show the variable importance estimated by RF with the entire data set. RF: RF out of cross-validation loop. cvRF: cross validation in the cross-validation loop 
A

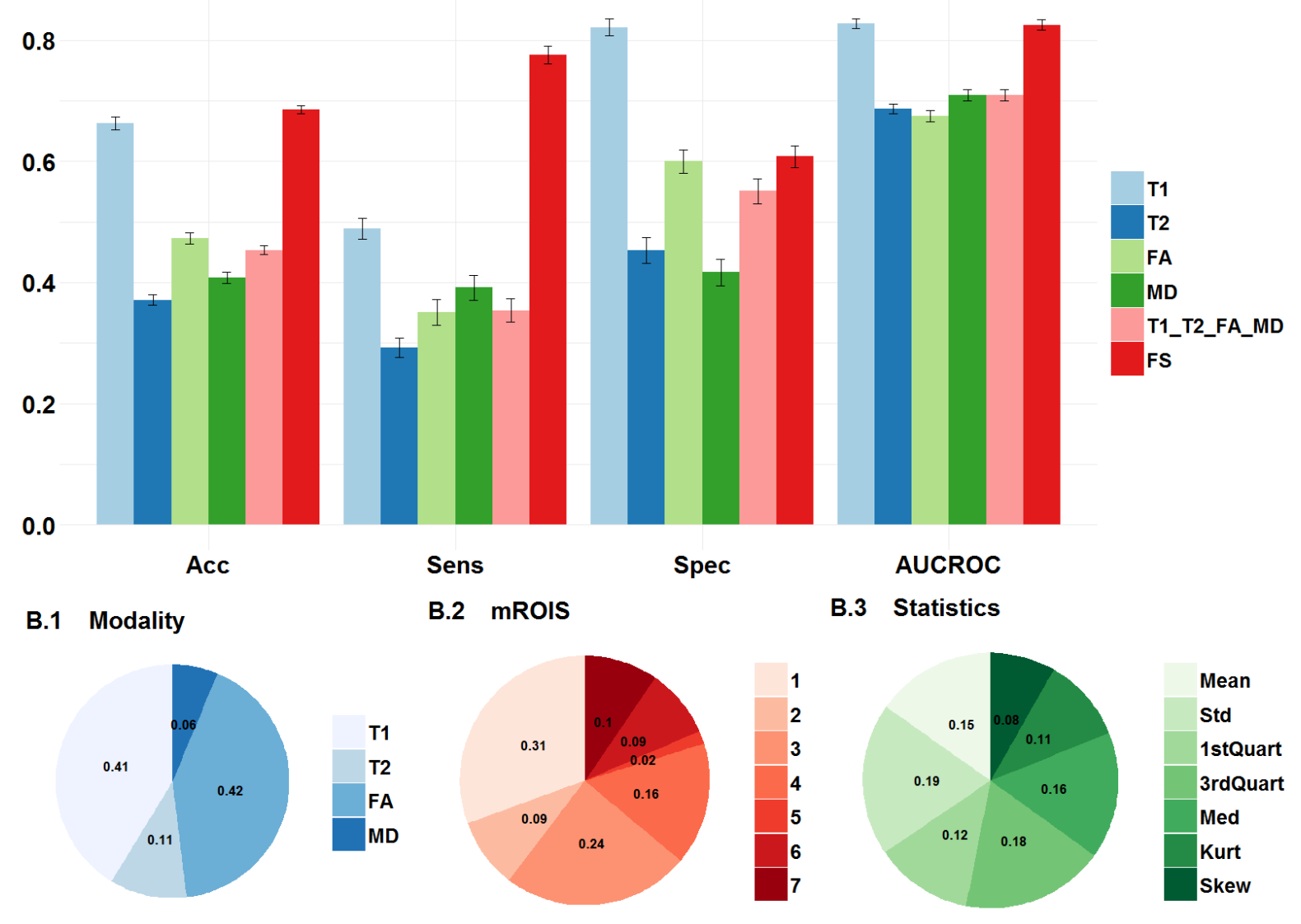

Figure 4.10 Performance of unimodal and multimodal SVM models to distinguish between animals in a one month continuous drinking paradigm from the same subjects after a one week of forced abstinence. (A) Performance in terms of. Accuracy (Acc), Sensitivity (Sens), specificity (Spec) and area under the ROC curve (AUCROC) of the SVM model. Contribution of selected features grouped according modality (B.1), mROI (B.2) and Statistics (B.3). T1: Model trained with T1-derived features; T2: Model trained with T2-derived features; FA: Model trained with FA-derived features; MD: Model trained with MD-derived features; T1_T2_FA_MD: Model trained with all features without feature selection. FS: Model trained with all features with feature selection.

Univariate models analyzed for the alcohol-abstinence discrimination (Table 4.3) showed comparable performance indices regardless of the modality, with T1 model showing the highest accuracy to discriminate states. However, the sensitivity of all of them was very low (Table 4.3 and Figure 4.10). As found in previous analysis, the best classification result is obtained with the multivariate model combined with feature selection, $69.9 \%$ of the cases are correctly assigned to either the alcohol or abstinent groups (being near to significance, $p=0.07$ ). From 20 features onwards, the model rapidly loses the overall ability to distinguish subjects after alcohol exposition 
(sensitivity) (Fig. 4.9B).

\begin{tabular}{|c|c|c|c|c|c|c|c|c|}
\hline \multirow[t]{2}{*}{ Models } & \multicolumn{2}{|c|}{ Accuracy } & \multicolumn{2}{|c|}{ Sensitivity } & \multicolumn{2}{|c|}{ Specificity } & \multicolumn{2}{|c|}{ AUC-ROC } \\
\hline & $\begin{array}{c}\text { Mean } \\
\text { (95\% C.I.) }\end{array}$ & p & $\begin{array}{c}\text { Mean } \\
\text { (95\% C.I.) }\end{array}$ & p & $\begin{array}{c}\text { Mean } \\
\text { (95\% C.I.) }\end{array}$ & $\mathbf{p}$ & $\begin{array}{c}\text { Mean } \\
\text { (95\% C.I.) }\end{array}$ & p \\
\hline $\mathrm{T} 1$ & $\begin{array}{c}0.66 \\
(0.48-0.77)\end{array}$ & 0.070 & $\begin{array}{c}0.49 \\
(0.28-0.67)\end{array}$ & 0.517 & $\begin{array}{c}0.82 \\
(0.56-1.00)\end{array}$ & 0.052 & $\begin{array}{c}0.83 \\
(0.76-0.93)\end{array}$ & 0.094 \\
\hline T2 & $\begin{array}{c}0.37 \\
(0.29-0.53)\end{array}$ & 0.862 & $\begin{array}{c}0.29 \\
(0.11-0.50)\end{array}$ & 0.726 & $\begin{array}{c}0.45 \\
(0.22-0.67)\end{array}$ & 0.588 & $\begin{array}{c}0.69 \\
(0.57-0.80)\end{array}$ & 0.536 \\
\hline FA & $\begin{array}{c}0.47 \\
(0.29-0.60)\end{array}$ & 0.576 & $\begin{array}{c}0.35 \\
(0.00-0.67)\end{array}$ & 0.761 & $\begin{array}{c}0.60 \\
(0.33-0.89)\end{array}$ & 0.309 & $\begin{array}{c}0.67 \\
(0.56-0.78)\end{array}$ & 0.565 \\
\hline MD & $\begin{array}{c}0.41 \\
(0.24-0.53)\end{array}$ & 0.846 & $\begin{array}{c}0.39 \\
(0.11-0.72)\end{array}$ & 0.630 & $\begin{array}{c}0.42 \\
(0.11-0.67)\end{array}$ & 0.658 & $\begin{array}{c}0.71 \\
(0.57-0.83)\end{array}$ & 0.392 \\
\hline $\mathrm{T} 1, \mathrm{~T} 2, \mathrm{FA}, \mathrm{MD}$ & $\begin{array}{c}0.45 \\
(0.36-0.53)\end{array}$ & 0.700 & $\begin{array}{c}0.35 \\
(0.11-0.67)\end{array}$ & 0.733 & $\begin{array}{c}0.55 \\
(0.22-0.89)\end{array}$ & 0.472 & $\begin{array}{c}0.71 \\
(0.59-0.83)\end{array}$ & 0.365 \\
\hline FS & $\begin{array}{c}0.70 \\
(0.58-0.77)\end{array}$ & 0.066 & $\begin{array}{c}0.78 \\
(0.61-0.89)\end{array}$ & 0.121 & $\begin{array}{c}0.61 \\
(0.33-0.78)\end{array}$ & 0.280 & $\begin{array}{c}0.83 \\
(0.70-0.95)\end{array}$ & 0.084 \\
\hline
\end{tabular}

Table 4.3 Performance of unimodal and multimodal SVM models to to distinguish between animals in a one month continuous drinking paradigm from the same subjects after a one week of forced abstinence. T1: Model trained with T1-derived features; T2: Model trained with T2-derived features; FA: Model trained with FA-derived features; MD: Model trained with MD-derived features; T1_T2_FA_MD: Model trained with all features without feature selection. FS: Model trained with all features with feature selection. P-values mean significance with respect to the label-permuted null model.

\section{Naltrexone treatment effect in alcohol exposed rats}

The next logic step in our analysis is to study the effect of the antagonist Naltrexone during the abstinence. For this purpose, multimodal features of exposed animals (positive class) and Naltrexone injected animals (negative class) are used to train the classification model that allow us distinguishing between both conditions. Detailed results are provided in the Figure 4.11 and Figure 4.12. Following the tendency of the previous results, FA-derived features contribute highly to the accuracy of the model with less contribution of T1-derived measures. T2 and MD are not selected as features contributing to the classification. From 5 features onwards, the model decreases progressively the performance mostly due to the loss of sensitivity (specificity decays slowly) (Fig. 4.11B). 


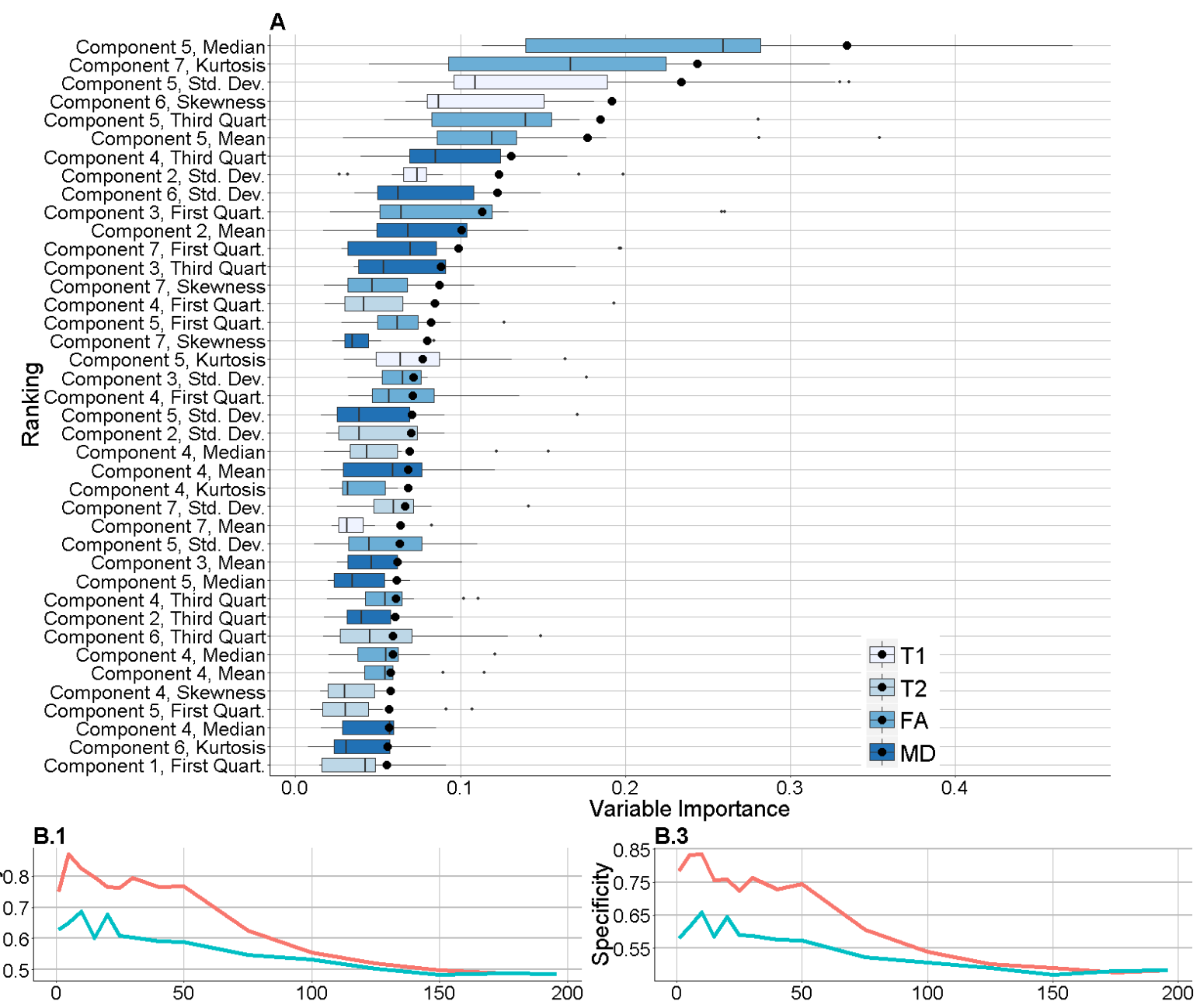

B. 2

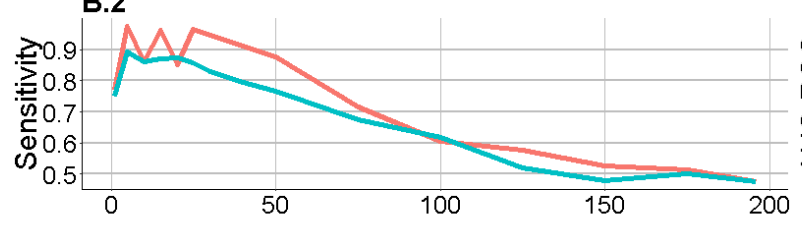

B. 4

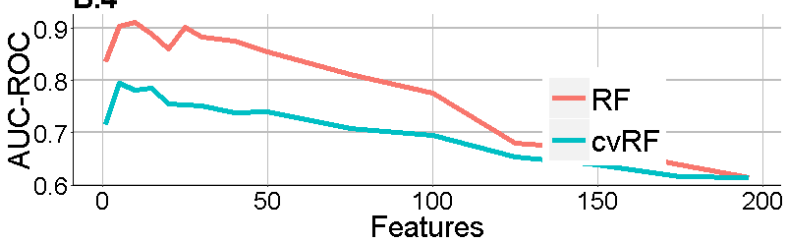

Figure 4.11 Results of the implemented model to distinguish between animals in a one month continuous drinking paradigm from the same subjects after a one week of naltrexone treatment. (A) Ranking of features according to their importance for model performance. Accuracy (B), Sensitivity (C), specificity (C) and area under the ROC curve (AUC) of the SVM model against the number of features (D). In figure (A) boxplots represent the distribution of variable importances across folds in the CV scheme. Larger dark points show the variable importance estimated by RF with the entire data set. RF: RF out of cross-validation loop. cvRF: cross validation in the cross-validation loop. 
Multivariate model without feature selection show a low performance, even lower than the univariate models (Table 4.4). As has been introduced above, from 16 features onwards the model provide poor results suggesting that noising features are introduced in the system affecting negatively to the prediction of the subjects injected with Naltrexone. Nevertheless, RF approach allows us discriminating the features which provide an accurate classification. Even although the specificity of the model is not especially high, the overall accuracy is $71.3 \%$.

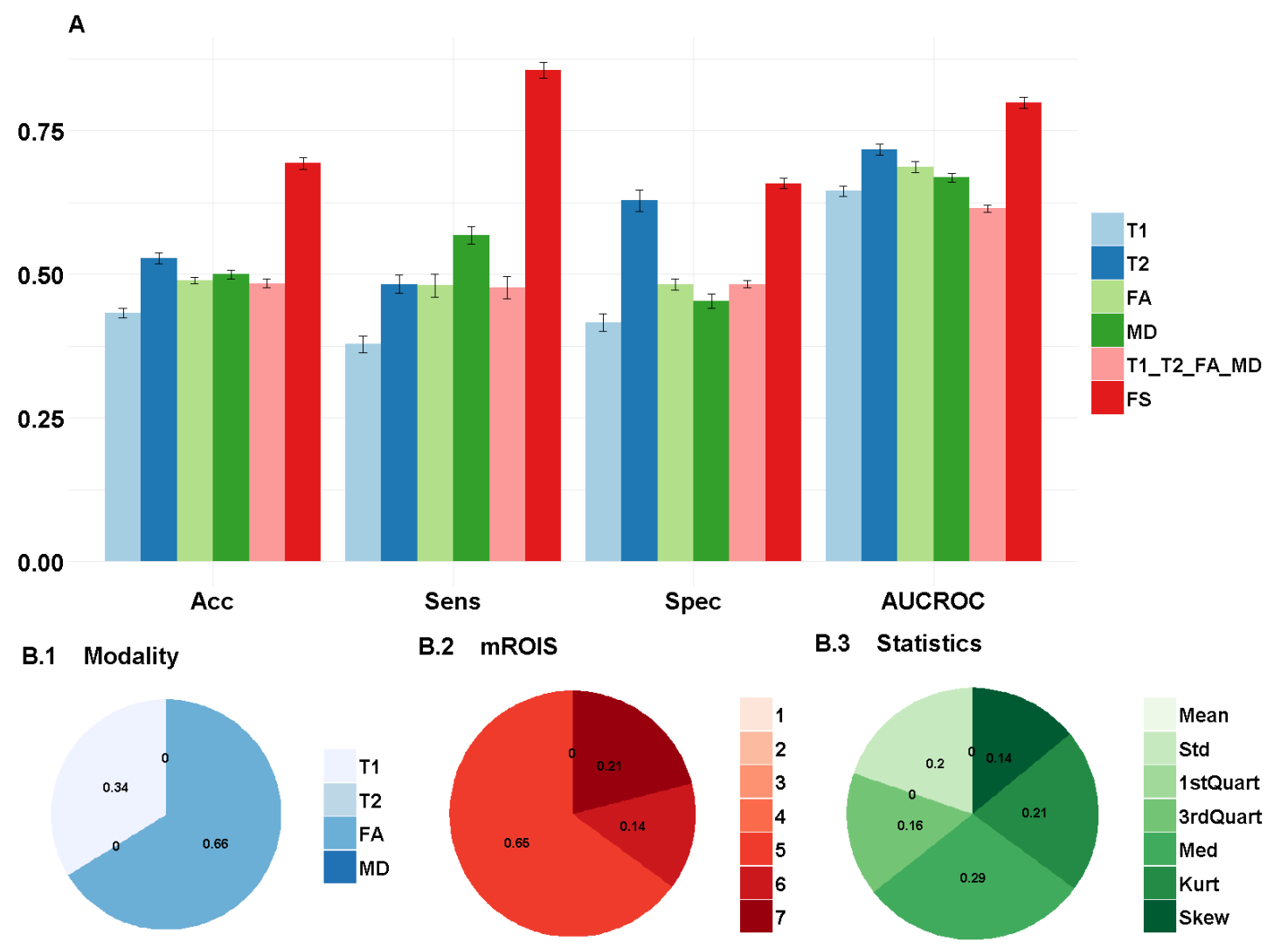

Figure 4.12 Performance of unimodal and multimodal SVM models to distinguish between animals in a one month continuous drinking paradigm from the same subjects after a one week of naltrexone treatment. (A) Performance in terms of. Accuracy (Acc), Sensitivity (Sens), specificity (Spec) and area under the ROC curve (AUCROC) of the SVM model. Contribution of selected features grouped according modality (B.1), mROI (B.2) and Statistics (B.3). T1: Model trained with T1-derived features; T2: Model trained with T2-derived features; FA: Model trained with FA-derived features; MD: Model trained with MD-derived features; T1_T2_FA_MD: Model trained with all features without feature selection. FS: Model trained with all features with feature selection. 


\begin{tabular}{|c|c|c|c|c|c|c|c|c|}
\hline \multirow{2}{*}{ Models } & \multicolumn{2}{|c|}{ Accuracy } & \multicolumn{2}{|c|}{ Sensitivity } & \multicolumn{2}{|c|}{ Specificity } & \multicolumn{2}{|c|}{ AUC-ROC } \\
\hline & $\begin{array}{c}\text { Mean } \\
\text { (95\% C.I.) }\end{array}$ & p & $\begin{array}{c}\text { Mean } \\
\text { (95\% C.I.) }\end{array}$ & p & $\begin{array}{c}\text { Mean } \\
\text { (95\% C.I.) }\end{array}$ & p & $\begin{array}{c}\text { Mean } \\
\text { (95\% C.I.) }\end{array}$ & $\mathbf{p}$ \\
\hline T1 & $\begin{array}{c}0.47 \\
(0.39-0.56)\end{array}$ & 0.577 & $\begin{array}{c}0.42 \\
(0.22-0.67)\end{array}$ & 0.751 & $\begin{array}{c}0.53 \\
(0.22-0.78)\end{array}$ & 0.513 & $\begin{array}{c}0.62 \\
(0.52-0.74)\end{array}$ & 0.743 \\
\hline T2 & $\begin{array}{c}0.58 \\
(0.39-0.72)\end{array}$ & 0.234 & $\begin{array}{c}0.48 \\
(0.22-0.67)\end{array}$ & 0.495 & $\begin{array}{c}0.68 \\
(0.33-0.89)\end{array}$ & 0.161 & $\begin{array}{c}0.72 \\
(0.56-0.93)\end{array}$ & 0.458 \\
\hline FA & $\begin{array}{c}0.50 \\
(0.39-0.61)\end{array}$ & 0.609 & $\begin{array}{c}0.63 \\
(0.44-0.89)\end{array}$ & 0.367 & $\begin{array}{c}0.37 \\
(0.11-0.67)\end{array}$ & 0.640 & $\begin{array}{c}0.70 \\
(0.56-0.81)\end{array}$ & 0.489 \\
\hline MD & $\begin{array}{c}0.47 \\
(0.39-0.56)\end{array}$ & 0.624 & $\begin{array}{c}0.50 \\
(0.22-0.89)\end{array}$ & 0.464 & $\begin{array}{c}0.45 \\
(0.11-0.67)\end{array}$ & 0.521 & $\begin{array}{c}0.65 \\
(0.59-0.74)\end{array}$ & 0.499 \\
\hline $\mathrm{T} 1, \mathrm{~T} 2, \mathrm{FA}, \mathrm{MD}$ & $\begin{array}{c}0.49 \\
(0.33-0.56)\end{array}$ & 0.559 & $\begin{array}{c}0.64 \\
(0.33-0.89)\end{array}$ & 0.308 & $\begin{array}{c}0.34 \\
(0.11-0.56)\end{array}$ & 0.699 & $\begin{array}{c}0.61 \\
(0.52-0.67)\end{array}$ & 0.821 \\
\hline FS & $\begin{array}{c}0.69 \\
(0.50-0.83)\end{array}$ & 0.050 & $\begin{array}{c}0.88 \\
(0.67-1.00)\end{array}$ & 0.011 & $\begin{array}{c}0.50 \\
(0.22-0.78)\end{array}$ & 0.579 & $\begin{array}{c}0.80 \\
(0.67-0.89)\end{array}$ & 0.149 \\
\hline
\end{tabular}

Table 4.4 Performance of unimodal and multimodal SVM models to distinguish between animals in a one month continuous drinking paradigm from the same subjects after a one week of naltrexone treatment. T1: Model trained with T1-derived features; T2: Model trained with T2-derived features; FA: Model trained with FA-derived features; MD: Model trained with MD-derived features; T1_T2_FA_MD: Model trained with all features without feature selection. FS: Model trained with all features with feature selection. P-values mean significance with respect to the label-permuted null model.

\section{A specific multimodal fingerprint for Naltrexone medicated brains}

In the final analysis of the study we push the discriminative capacity of our method to differentiate between two abstinence conditions of 1 week duration each. In the second condition animals received daily treatment with the opioid receptor antagonist naltrexone, at doses known to efficiently decrease compulsive drinking (naltrexone condition). Naltrexone treated rats were defined as positive class. Results are shown in Figure 4.13. Analysis was performed excluding the testing sets from (distribution of importance for each fold and repetition are shown as boxplots in $A$ and classification performances are depicted as blue lines in B.1-4) and including it in the features selection step (large black dots in A and red lines in B.1-4). 


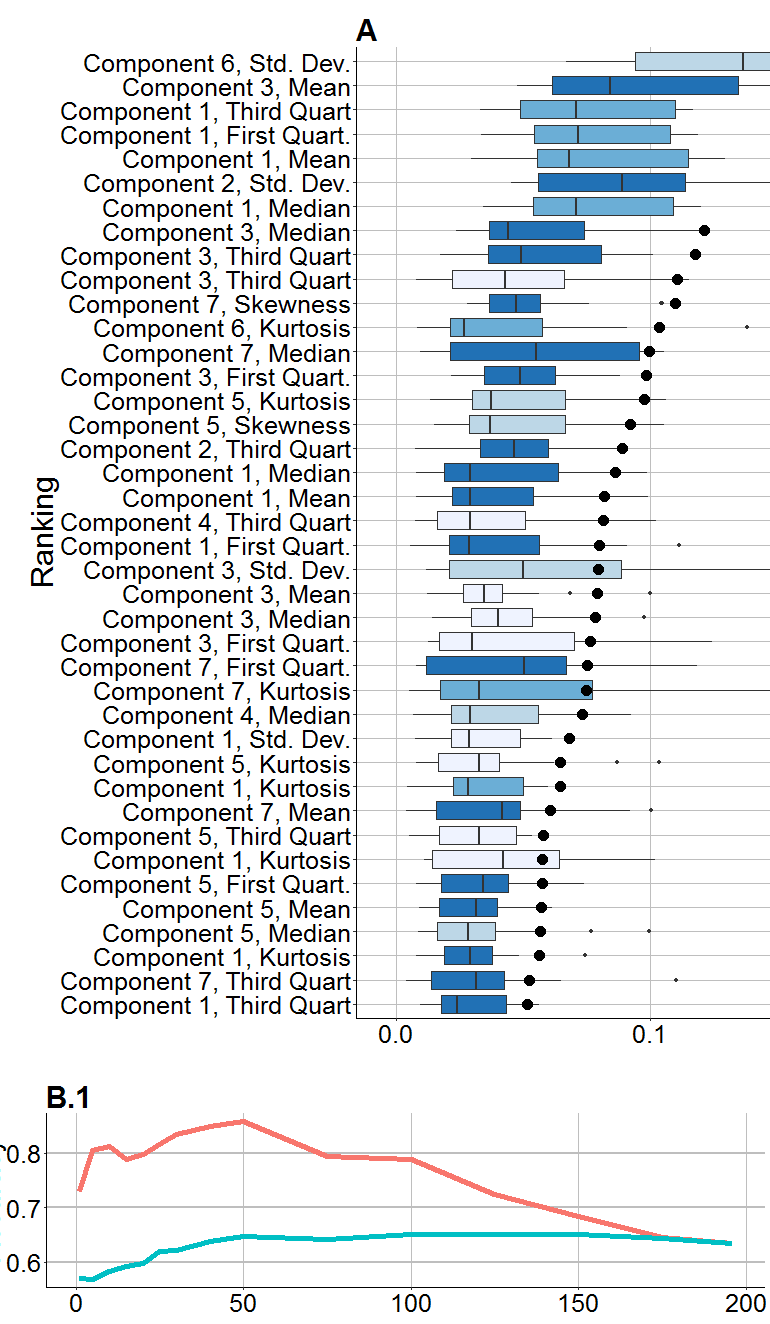

B.2

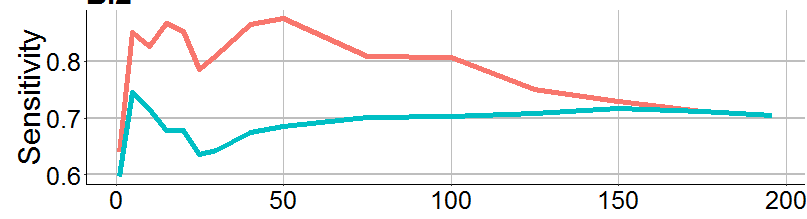

Variable Importance

B.3

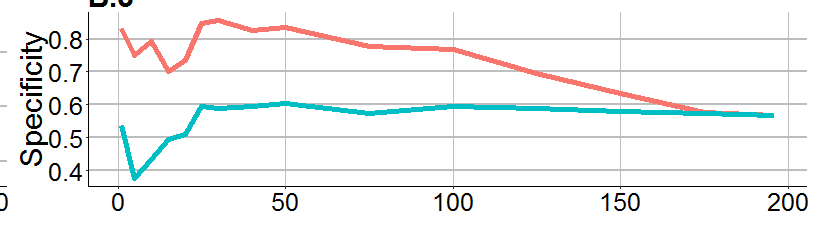

B.4

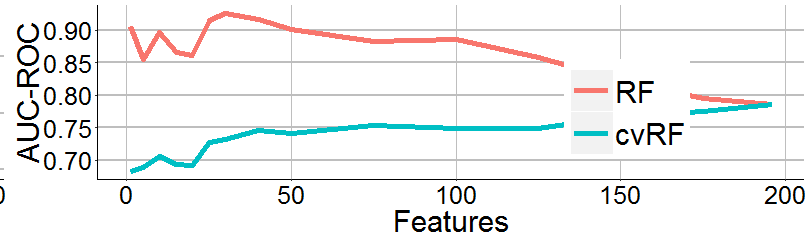

Figure 4.13 Feature selection to classify abstinent subjects according to Naltrexone or saline treatment. (A) Ranking of features according to their importance for model performance. Accuracy (B), Sensitivity (C), specificity (C) and area under the ROC curve (AUC) of the SVM model against the number of features (D). In figure (A) boxplots represent the distribution of variable importances across folds in the CV scheme. Larger dark points show the variable importance estimated by RF with the entire data set. RF: RF out of crossvalidation loop. cvRF: cross validation in the cross-validation loop.

The inclusion of the testing samples produces a significant increase of the performance possibly due to the so-called overfitting and therefore, reducing the generalization of the model. In terms of higher accuracy, the optimal number of features in this particular analysis is 100 (50 if 
testing set is not part of RF input), with all imaging modalities contributing to them, being once again MD derived measures especially well represented (Fig. 4.13A and 4.14B). T1, T2 and FA measures contribute quite homogeneously to predict whether the subject has been injected with Naltrexone or has not.

Figure 4.14 shows the performance of the unimodal and multimodal (with and without feature selection) models. Feature selection was performed in the inner loop of the cross validation scheme in order to minimize overfitting. Univariate models show a significant reduction in almost all measures of classification performance when compared to multivariate models (Table 4.5). The univariate MD model was the less imprecise (61\% of Accuracy), as expected from the high ranking of MD-derive measures in Fig.4.14B1. Feature selection improved all performance indices and allows multivariate models to reach a 65\% (against the 63\%) of samples correctly classified with an AUC of $70 \%$ (Table 4.5). Accuracy of the multimodal model, even though no significant, presents a clear tendency to be above the chance $(p=0.07)$.

Exploring the contribution of the features, MD-derived features contribute the most $(40 \%)$ with equal contribution of the remaining modalities (20\%). This is reflected by a cv-accuracy of $65 \%$ of the MD-derived classifier (MSMCA). mROIS 1 and 3 contributes with 24\% and 19\% of the importance respectively. 
A

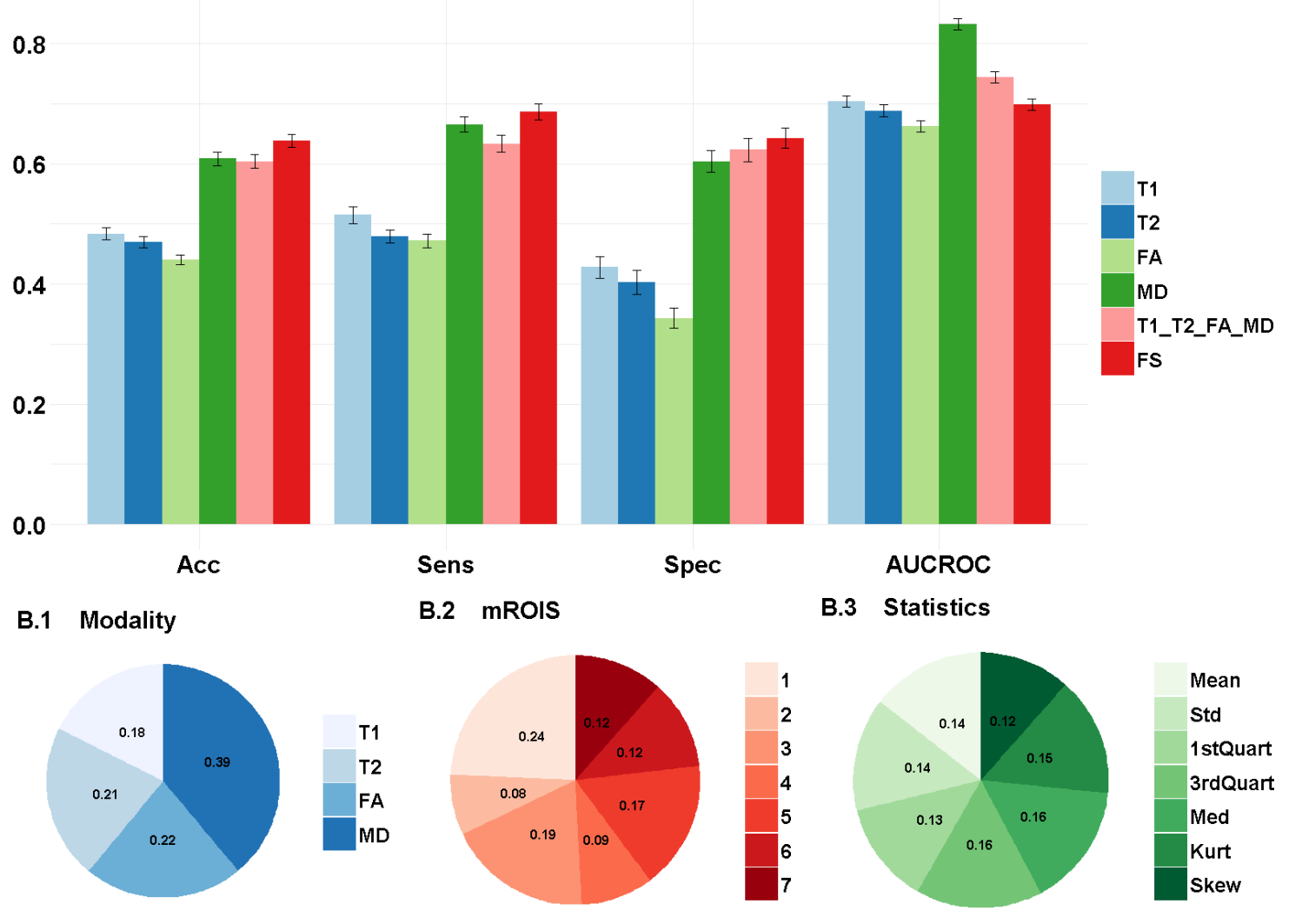

Figure 4.14 Performance of unimodal and multimodal SVM models to classify abstinent subjects according to Naltrexone or saline treatment. (A) Performance in terms of. Accuracy (Acc), Sensitivity (Sens), specificity (Spec) and area under the ROC curve (AUCROC) of the SVM model. Contribution of selected features grouped according modality (B.1), mROI (B.2) and Statistics (B.3). T1: Model trained with T1-derived features; T2: Model trained with T2-derived features; FA: Model trained with FA-derived features; MD: Model trained with MD-derived features; T1_T2_FA_MD: Model trained with all features without feature selection. FS: Model trained with all features with feature selection. 


\begin{tabular}{|c|c|c|c|c|c|c|c|c|}
\hline \multirow[t]{2}{*}{ Models } & \multicolumn{2}{|l|}{ Accuracy } & \multicolumn{2}{|c|}{ Sensitivity } & \multicolumn{2}{|c|}{ Specificity } & \multicolumn{2}{|c|}{ AUC-ROC } \\
\hline & $\begin{array}{c}\text { Mean } \\
\text { (95\% C.I.) }\end{array}$ & p & $\begin{array}{c}\text { Mean } \\
\text { (95\% C.I.) }\end{array}$ & p & $\begin{array}{c}\text { Mean } \\
\text { (95\% C.I.) }\end{array}$ & p & $\begin{array}{c}\text { Mean } \\
\text { (95\% C.I.) }\end{array}$ & p \\
\hline T1 & $0.52(0.37-0.66)$ & 0.297 & $0.59(0.33-0.78)$ & 0.303 & $0.45(0.11-0.72)$ & 0.541 & $0.71(0.57-0.85)$ & 0.496 \\
\hline T2 & $0.49(0.34-0.63)$ & 0.329 & $0.66(0.44-0.89)$ & 0.241 & $0.30(0.00-0.56)$ & 0.853 & $0.69(0.56-0.81)$ & 0.557 \\
\hline FA & $0.44(0.31-0.58)$ & 0.557 & $0.59(0.33-0.89)$ & 0.334 & $0.28(0.00-0.56)$ & 0.888 & $0.67(0.52-0.82)$ & 0.623 \\
\hline MD & $0.61(0.42-0.71)$ & 0.090 & $0.67(0.33-0.78)$ & 0.291 & $0.54(0.22-0.78)$ & 0.461 & $0.83(0.70-0.93)$ & 0.160 \\
\hline $\mathrm{T} 1, \mathrm{~T} 2, \mathrm{FA}, \mathrm{MD}$ & $0.63(0.41-0.82)$ & 0.086 & $0.70(0.44-0.89)$ & 0.150 & $0.57(0.28-0.78)$ & 0.339 & $0.79(0.65-0.89)$ & 0.250 \\
\hline FS & $0.65(0.48-0.77)$ & 0.070 & $0.70(0.56-0.89)$ & 0.143 & $0.59(0.28-0.78)$ & 0.338 & $0.75(0.61-0.89)$ & 0.353 \\
\hline
\end{tabular}

Table 4.5 Performance of unimodal and multimodal SVM models to distinguish abstinent subjects according to Naltrexone or saline treatment. T1: Model trained with T1-derived features; T2: Model trained with T2-derived features; FA: Model trained with FA-derived features; MD: Model trained with MDderived features; T1_T2_FA_MD: Model trained with all features without feature selection. FS: Model trained with all features with feature selection. P-values mean significance with respect to the label-permuted null model.

\section{Validating the framework}

As has been pointed above, feature selection improves the classification performance removing noising features and identifying feature patterns. One of the main aims of this work was to identify a subset of features which better discriminates two experimental stages. We state that variable importance calculated by RF provides reliable importance quantification. In order to validate such relationship we test variable importances against the Null distribution of variable importances obtained by 1000 label-permutation. We found a significant negative correlation between the obtained $p$-values and variable importance for all possible classifications (Spearman rank correlation $r<-0.95, p<0.001$ ) (Fig 4.15). 

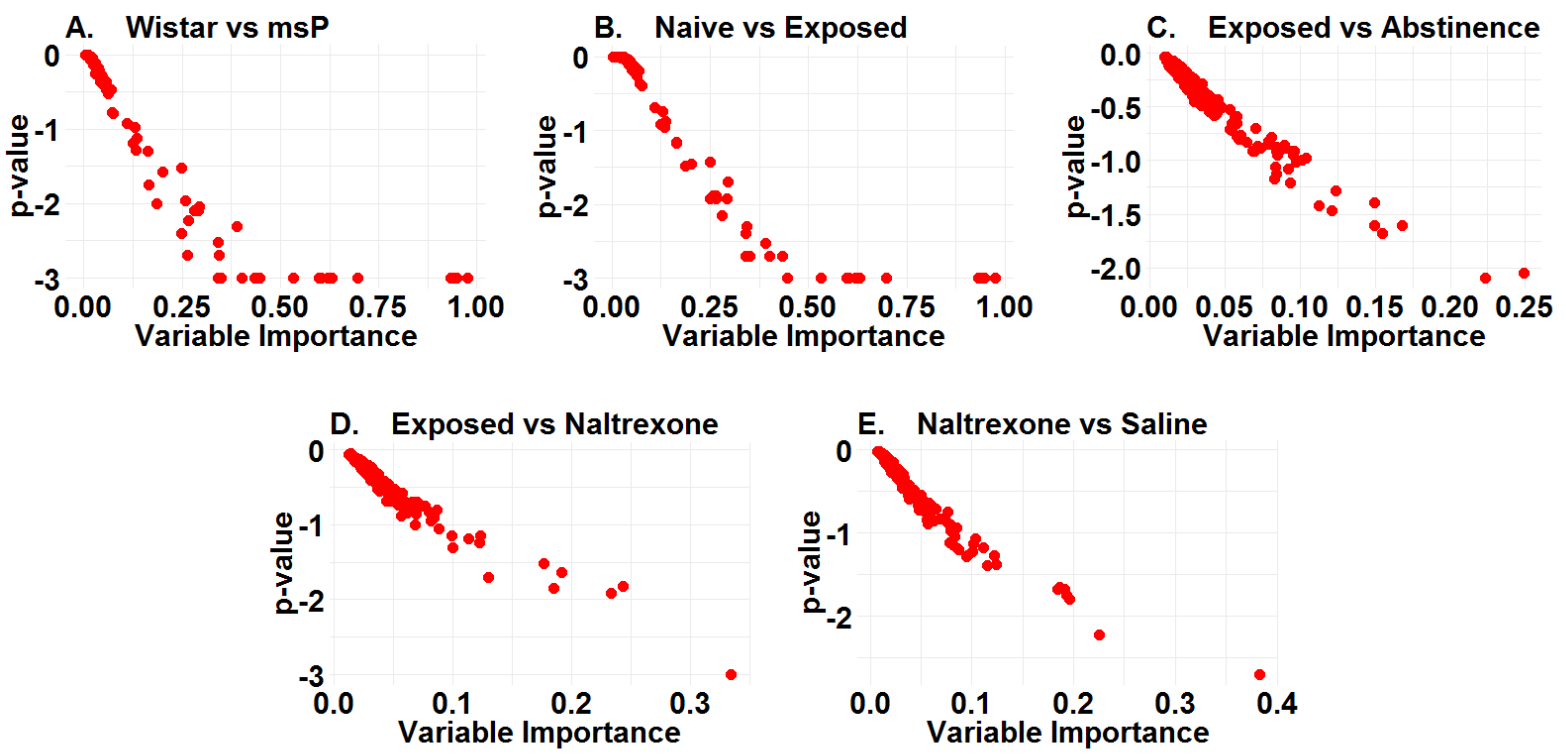

Figure 4.15 Relationship between variable importance and label-permutation tests. Variable importances for discriminating Wistar vs msP rats (A), Naïve vs Exposed (B), Exposed vs Abstinent rats (C), Exposed vs Naltrexone-treated rats (D) and Saline vs Naltrexone-treated rats (E). Significant Spearman rank correlations were significant for all the relationships $(p<0.001)$. $p$-values are shown in logarithmic scales. Note that for 1000 permutations, the $p$ resolution is 0.001 ( -3 after logarithmic transformation).

Moreover, one of the major challenges in $\mathrm{ML}$ is the reduction of the overfitting in modest data set sizes. Generalization of our results was investigated by gold standard cross-validation techniques by including or excluding feature selection steps in the inner loop. Of note, the latter introduces deliberately overfitting. Results are depicted in Figure 4.16. A higher classification performance is obtained when the feature selection is performed independently from classification. However, this procedure might increase the overfitting risk given testing set is partly influencing the feature selection outcome in different extents in each of the classification tasks. 

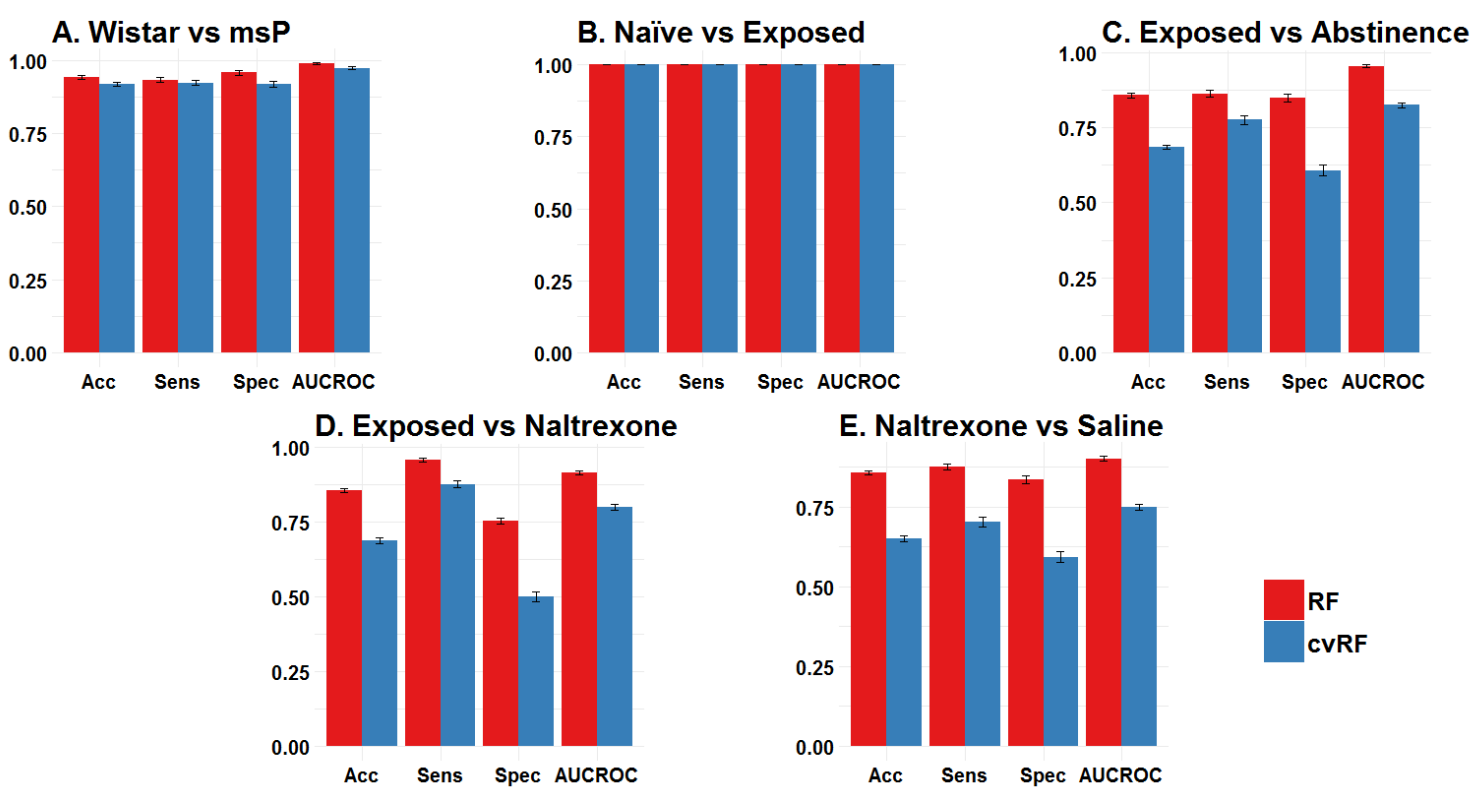

E. Naltrexone vs Saline

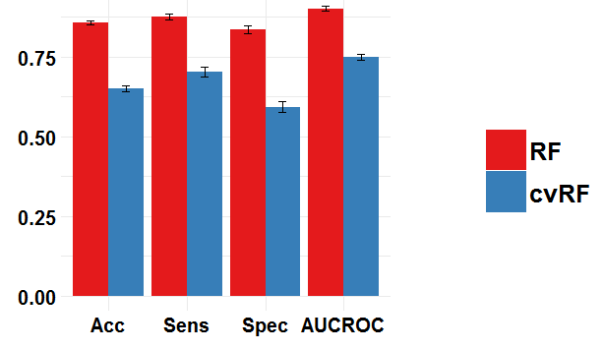

Figure 4.16 Effect of inclusion of RF feature selection in the inner loop of the cross-validation scheme. Performance of the multimodal SVM models to differentiate Wistar vs msP rats (A), Naiive vs Exposed (B), Exposed vs Abstinent rats (C), Exposed vs Naltrexone-treated rats (D) and Saline vs Naltrexone-treated rats (E).

\subsubsection{Discussion}

The main findings of the present section are that: (1) robust and anatomically meaningful multimodal signatures, here called mROIs, capturing the microstructural and biochemical properties of brain tissue can be defined by using MRI and finite mixture models, (2) the utility of these multimodal signatures to characterize disease stages, here called MSDs, in a model of alcohol use disorder has been demonstrated, (3) the results show that even moderate phenotypes of alcohol drinking leave distinct recognizable signatures in brain tissues, (4) alterations in brain parenchyma induced by alcohol persist into abstinence, at least in its earlier stages, and (5) further changes in tissue microstructure are demonstrated after naltrexone treatment in alcohol abstinent animals, defining a treatment-related brain signature for this anti-relapse medication.

We have presented an analytical framework that uses tissue microstructural information extracted from different MRI modalities to implement novel tissue segmentation into multimodal categories or mROls. Combined with feature ranking obtained by RF and SVM learning models, 
defined mROIs attained an extremely good performance in the discrimination of different alcohol drinking stages. One novel aspect of our method that largely contributed to the success of the classifier is that the distribution of the different MRI parameters within every $\mathrm{mROI}$ is fully described by a number of up to third-order statistics in order to describe properly the inhomogeneous microstructure within an mROI. Indeed, the first positions in the ranking of variable importance for correct classifications were usually occupied by features that describe the statistical distribution of the parameters rather than mean. Between the different modalities, our results highlight the contribution of MD to properly characterize different stages. As such, MD can be seen as a potentially relevant biomarker. Nevertheless multimodal description increases the predictive power up to $11 \%$. From a neurobiological perspective, the developed methodology provides spatial locations (mROIs) and quantitates alterations in a number of parameters describing tissue microstructure in specific disease stages and therefore may also assist further investigations to fill the gap between clinical observations and neurobiological mechanisms underlying such alterations enabling accurate classifications.

Analytical approaches based on FFM have been traditionally used in neuroimaging studies considering a brain composition limited to three types of tissue (gray and white matter and cerebrospinal fluid) and combinations of them. However, in the present work we bring a new concept in tissue classification based on the combination of multiple microstructural properties of brain tissues that define novel tissue categories. This approach maximizes the potentialities of multimodal MRI providing comprehensive and non-invasive descriptions of brain tissue without including prior spatial information. The utility of this multimodal descriptions or signatures to identify and characterize pathological conditions or disease stages, that we have called MSDs, has been explored in a model of alcohol consumption in rats. Our results provide a strong support for the presented framework. We propose that neuropsychiatric evaluations assisted by human MISDs could be valuable for accurate diagnosis and prognosis.

We have visually shown with a reduced representation of the multimodal space that the acquired brain MRI parameters segregate according to the experimental conditions (Fig. 4.3A). This is especially clear for the naïve state which totally separates from the alcohol exposed conditions (no overlap between confidence ellipses in the PCA), in perfect agreement with the results of the 
classification model (100\% of the cases are correctly assigned) (Fig. 4.7B). A challenging discrimination was found between alcohol-drinking and abstinence. Both abstinence conditions (Naltrexone and non-treated) were discriminated with an accuracy around $70 \%$ and better identification of abstinence-related samples (sensitivity). Namely, that the microstructural changes in brain parenchyma induced by alcohol drinking are largely persistent after alcohol withdrawal in this relapse-prone sate. Interestingly, although confidence ellipses of both abstinent conditions partially overlap, the simple PCA applied to the longitudinal imaging study already suggested a tendency for naltrexone treated subjects to return towards a baseline (naïve) state, while saline treated abstinent animals evolve towards a new state. This divergence between saline and naltrexone treated abstinent states was confirmed by our analytical model, with both conditions being correctly discriminated with an accuracy of $65 \%$ and demonstrating that the treatment with this anti-relapse medication might induce a physical change in brain parenchyma.

An important confounding effect is the age and brain modifications occurring in parallel to the alcohol consumption. As it will be demonstrated in the following section (Section 4.3.1), despite its indubitable presence, observed effects are mainly due to alcohol rather than ongoing brain maturation.

In comparison to other studies, our findings resulted in enhanced predictive power between $4 \%$ (for treatment and alcohol exposure) and $11 \%$ in discriminating abstinence periods respect BSMCA. These results clearly surpass increases found in Alzheimer's diseases or schizophrenia with modest improvements between 3 and 7\% (132-135). All these results together evidence the enhanced sensitivity of multimodal and multivariate approaches in the identification of MRI signatures associated to multiple pathological stages. 


\subsection{A mesoscopic multimodal machine learning approach from alcohol initiation to treatment in $\mathrm{msP}$ rats}

\subsubsection{Effect of alcohol consumption in MRI parametric maps}

Voxelwise comparison revealed widespread changes produced by a month of continuous $10 \% \mathrm{EtOH}$ consumption in different parametric maps independent of natural brain changes (Figure 4.17, left). For the sack of simplicity and given the full description of unimodal changes are out of the scope of this work, they will not describe them in detail. Our ROI-wise analysis confirmed such results changes (Figure 4.17, right). There was a significant interaction effect Group x ROI in T1 values, $F=10.01, p<1 e-5$. A significant trend in group effect was found $(F=3.99,0.054)$. Bonferroni post-hoc tests revealed significant differences in cingulate $(p=0.008)$ and insular cortex $(p=0.001)$. There was a significant interaction effect Group x ROI $(F=6.98, p<0.004)$ and group main effect $(F=10.19, p<0.003)$ in T2 relaxation times. Bonferroni post-hoc tests revealed significant differences in nucleus accumbens $(p=0.001)$, caudate-putamen $(p<1 e-5)$, cingulate $(p<0.001)$, hippocampus $(p<1 e-4)$ and primary somatosensory cortex $(p<1 e-5)$. There was a significant interaction effect Group x ROI $(F=9.15, p<1 e-4)$ and group main effect $(F=4.94, p=0.03)$ in FA. Bonferroni post-hoc tests revealed significant differences in primary somatosensory cortex $(p=0.01)$ and insular cortex $(p<0.001)$. There was a significant interaction effect Group $x$ ROI $(F=3.10, p<0.046)$ and group main effect $(F=10.19, p<0.003)$ in MD values. Bonferroni post-hoc tests revealed significant differences in nucleus accumbens $(p<1 e-4)$, caudate-putamen $(p<1 e-8)$, cingulate $(p<1 e-4)$, hippocampus $(p<1 e-4)$ and primary somatosensory cortex $(p<1 e-8)$ and insular cortex $(p<1 e-4)$. Of note MD changes produced by alcohol consumption were systematically opposite to those changes of normal aging in rat brain. Similar effects were found in other MRI parameters. By contrast, changes in T2 relaxometry maps were decreased in higher degree due to heavy alcohol consumption. 
A

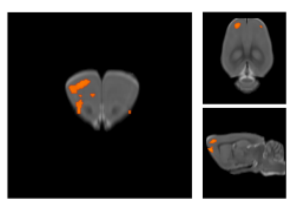

B

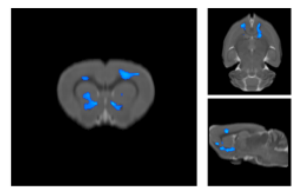

C
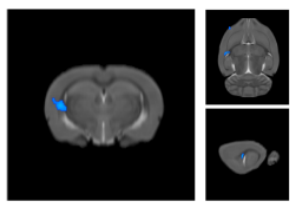

D
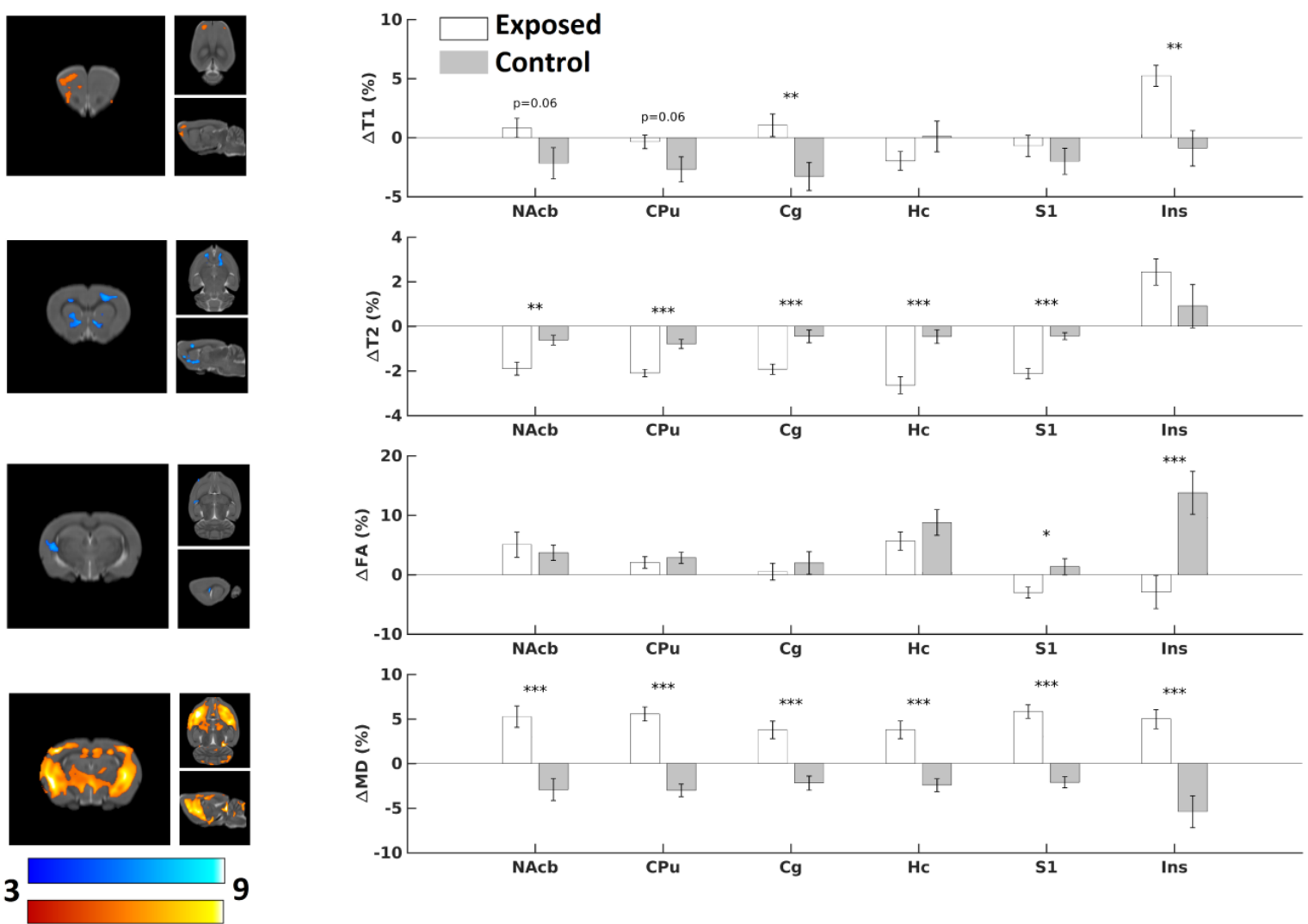

Figure 4.17 Effect of alcohol consumption in msP rats compared of one month of isolation in the home-cage for controlling age effects $(\mathbf{n = 1 7 - 1 8 )}$. Voxelwise inferences of differences in T1 maps (A), T2 maps (B), FA (C) and MD (D) between exposed and control msP rats (left) and differences in selected ROIS (right). Statistical maps were thresholded with pFWE<0.05. Nacb: nucleus accumbens; $\mathrm{CPu}$ : caudate-putamen; Cg: Cingulate cortex; Hc: hippocampus; S1: primary somatosensory cortex; Ins: insular cortex; CC: corpus callosum; FA: fractional anisotropy; MD: mean diffusivity. ${ }^{*} p<0.05,{ }^{* *} p<0.01, * * *_{p}<0.001$.

\subsubsection{Variable Importance}

Having probed that alcohol consumption affect MRI parameters independent of normal aging, our first aim was to investigate the local importance of each of the MRI measures in the ability to discriminate naïve and exposed states in msP rats. Random Forest algorithm was implemented to compute the relevance of each modality. The ranked features fed forwardly voxel-based SVM models. As depicted in Figure 4.18, feature importance confirmed a heterogeneous spatial profile of 
importance reflecting the locally diverse nature of microstructural neuroadaptations produced by a month of heavy alcohol consumption. Importance spatial profiles largely reproduce results of the single MRI modality statistical analysis. Overall, MD and T2 relaxometry values showed higher relevancies as discrimination predictors. It is noteworthy to point the importance of brain morphometry related measures such as grey and white matter volumes also seem to play a fundamental role in the discrimination of the experimental states likely due to the ongoing brain maturation during the ethanol exposition. More detailed information about the spatial profiles is shown in Fig. 4.18 and Table 4.6. 


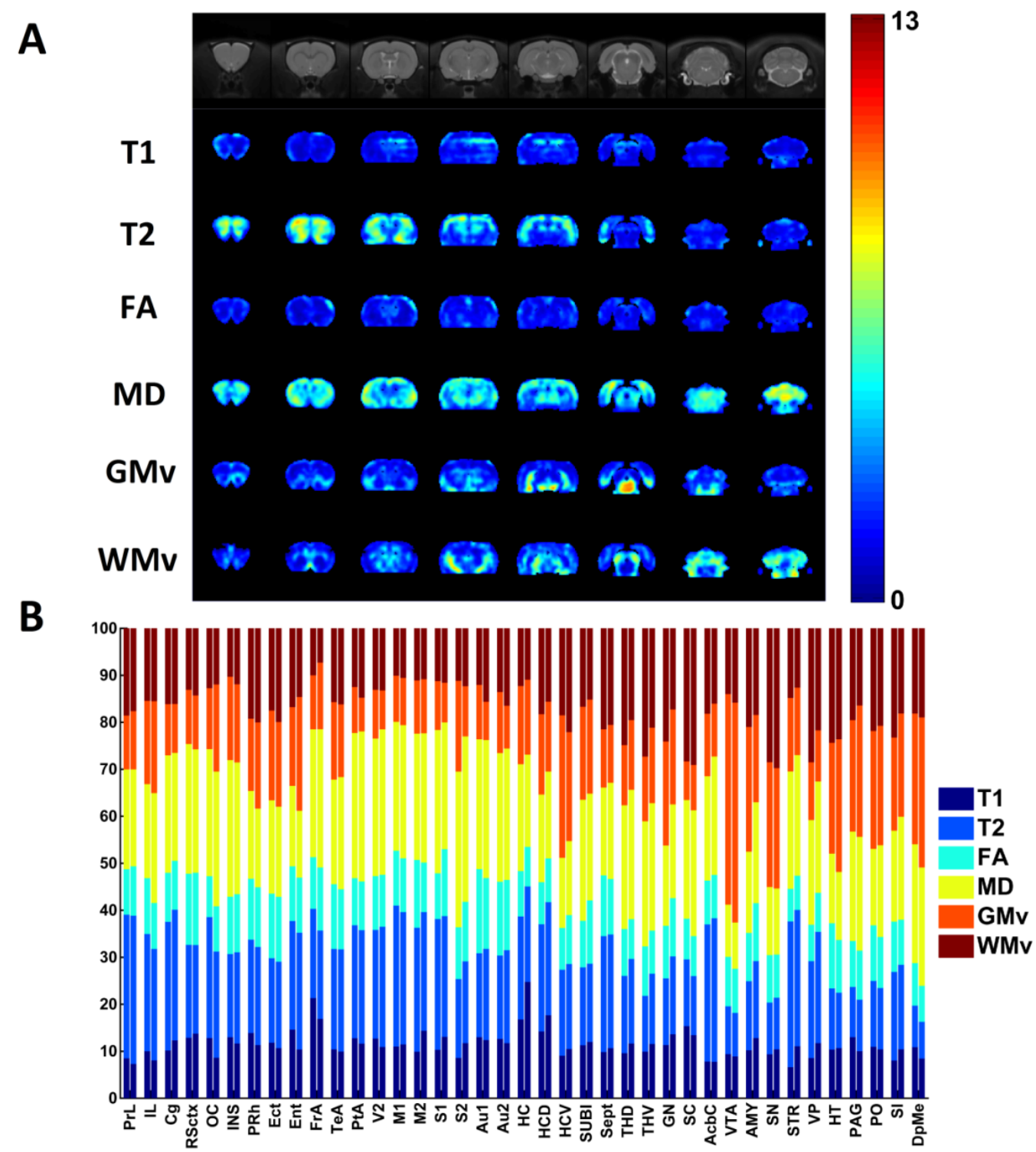

Figure 4.18 Importance of IMSD in discriminating Naïve and Exposed experimental states. (A) From posterior to anterior slices showing the importance of each modality forming IMSD. From top to down, anatomical reference, $\mathrm{T} 1, \mathrm{~T} 2$ relaxation maps, $\mathrm{FA}, \mathrm{MD}$, gray matter and white matter volume. (B) Contribution in percentage to each modality to discriminate experimental states for 39 anatomical regions. 
Variable Importance

\begin{tabular}{lllllll|lllllll}
\hline \hline & T1 & T2 & FA & MD & GMv & WMv & & T1 & T2 & FA & MD & GMv & WMv \\
\hline \hline PrL & 1.4 & 5,4 & 1.74 & 3.65 & 2.06 & 3.26 & HCD & 2.64 & 3.99 & 1.54 & 3.14 & 2.76 & 2.88 \\
IL & 1.58 & 4.26 & 1.88 & 3.72 & 3.18 & 2.66 & HCV & 1.6 & 2.98 & 1.53 & 2.43 & 4.35 & 3.12 \\
Cg & 1.96 & 4.82 & 1.83 & 4.15 & 1.86 & 2.85 & SUBI & 1.82 & 2.61 & 1.83 & 3.9 & 3.15 & 2.54 \\
RSctx & 2.14 & 3.17 & 2.44 & 4.22 & 1.82 & 2.14 & Sept & 1.77 & 4.14 & 2.14 & 3.44 & 2.16 & 3.67 \\
OC & 1.76 & 4.26 & 1.54 & 4.75 & 2.72 & 2.09 & THD & 1.83 & 2.92 & 1.6 & 4.68 & 2.39 & 3.99 \\
INS & 1.89 & 2.9 & 1.91 & 4.56 & 2.76 & 1.75 & THV & 1.87 & 2.28 & 1.72 & 4.59 & 2.6 & 4.4 \\
PRh & 1.97 & 3.28 & 1.99 & 2.75 & 2.56 & 3.06 & GN & 2.01 & 2.45 & 1.93 & 2.93 & 3.46 & 3.32 \\
Ect & 1.77 & 2.84 & 2.13 & 3 & 2.9 & 2.93 & SC & 2.43 & 2.23 & 1.47 & 4.46 & 1.6 & 5.03 \\
FrA & 2.96 & 3.12 & 1.91 & 4.79 & 2.03 & 1.36 & AcbC & 1.22 & 4.74 & 1.45 & 3.78 & 1.91 & 2.7 \\
TeA & 1.56 & 3.32 & 2.1 & 3.51 & 2.66 & 2.49 & VTA & 1.34 & 1.41 & 1.48 & 1.53 & 6.74 & 2.14 \\
PtA & 1.89 & 4.09 & 1.58 & 5.15 & 1.33 & 2.18 & AMY & 1.74 & 2.26 & 1.7 & 2.89 & 3.33 & 2.91 \\
V2 & 1.81 & 4 & 1.72 & 4.83 & 1.49 & 2.14 & SN & 1.3 & 1.44 & 1.24 & 1.86 & 3.3 & 3.78 \\
M1 & 1.74 & 5.02 & 1.77 & 4.69 & 1.53 & 1.76 & STR & 1.56 & 5.19 & 1.24 & 4.39 & 2.61 & 2.41 \\
M2 & 1.81 & 4.47 & 1.96 & 4.5 & 1.78 & 1.85 & VP & 1.72 & 3.57 & 1.34 & 3.8 & 1.98 & 4.22 \\
S1 & 1.96 & 4.56 & 1.91 & 4.86 & 1.52 & 1.99 & HT & 1.49 & 1.78 & 1.81 & 2.03 & 3.75 & 3.39 \\
S2 & 1.62 & 2.79 & 1.87 & 5.57 & 2.54 & 1.95 & PAG & 1.89 & 1.74 & 1.61 & 3.92 & 4.37 & 2.85 \\
Au1 & 1.94 & 2.94 & 2.6 & 4.63 & 1.64 & 2.26 & PO & 1.4 & 1.66 & 1.46 & 2.15 & 3.29 & 2.73 \\
Au2 & 1.91 & 3.02 & 2.45 & 4.47 & 1.82 & 2.52 & SI & 1.42 & 2.62 & 1.56 & 3.05 & 3.21 & 3.13 \\
HC & 3.5 & 3.56 & 1.53 & 3.56 & 2.78 & 2.05 & DpMe & 1.62 & 1.39 & 1.43 & 4.24 & 5.26 & 3.05 \\
\hline
\end{tabular}

Table 4.6Variable importance of the MRI maps. Values represent the average Gini variable importance in 38 anatomical regions.

As expected, variable importance maps assessed by RF reproduces those maps obtained by univariate statistical approaches. High RF importances are associated to high absolute $t$ values. Specifically, several cortical and subcortical regions showed differences between Naïve and postEtOH exposed states in the univariate analysis of the different MRI parametric maps (Fig. 4.19). There is an overall increase in MD and an overall decrease in T2. Structural measurements as GM and WM volume were mostly increased. However, GMv in the prelimbic (PL) and Somatosensory cortex ( $S 1$ ) were reduced ( $t=4.26, p=0.001$ and $t=3.85, p<0.001$, respectively). 

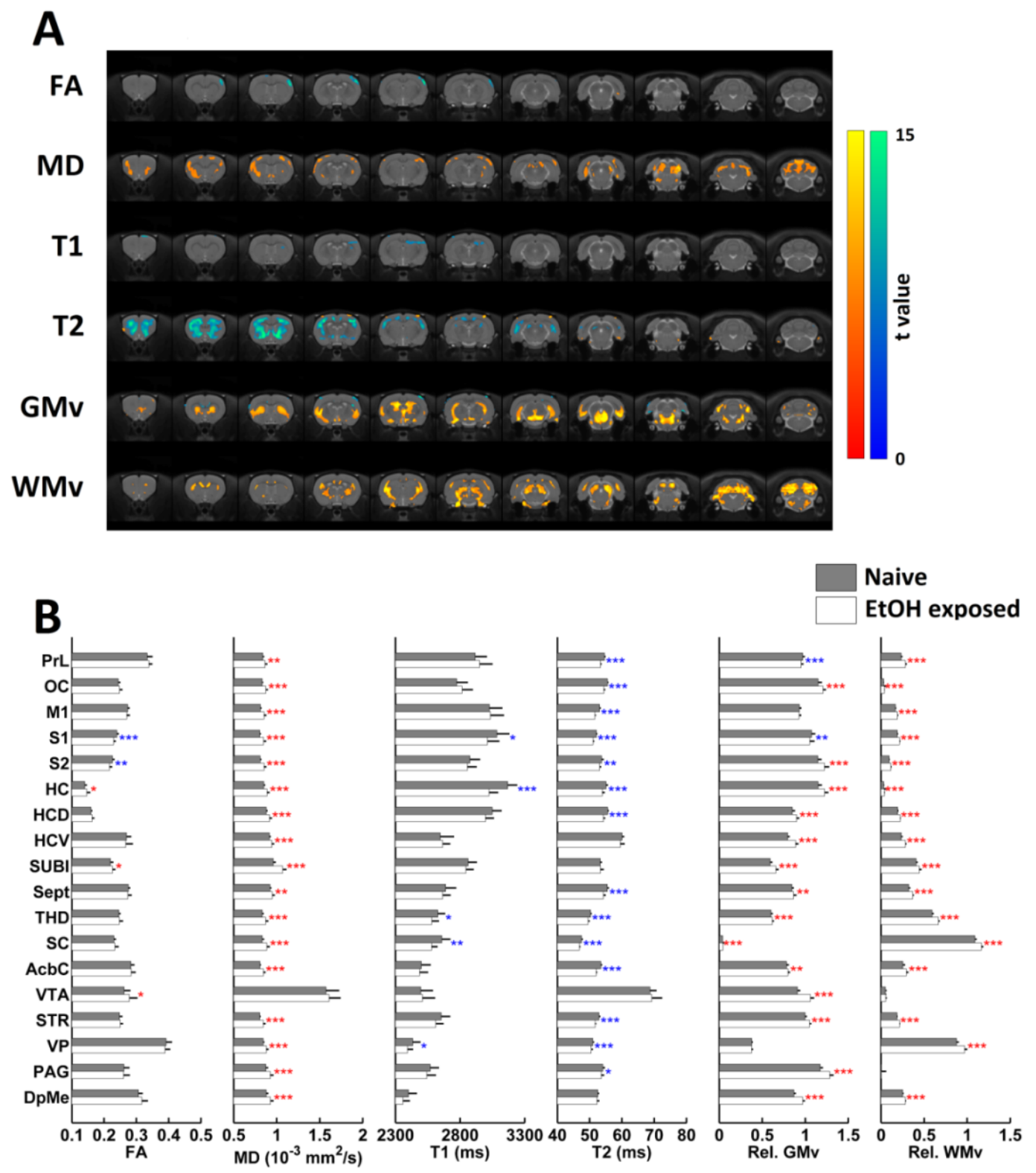

Figure 4.19 Spatial representation of tissue parameter differences between naïve and post-alcohol exposure state. (A) Significant differences in multimodal MRI parametric maps produced by one month of two bottles free choice paradigm ( $p_{\mathrm{FWE}}<0.05, \mathrm{p}_{\text {cluster }}<0.05$ ) (B) Comparisons of the MRI parameters in anatomical areas showing significant differences in any parametric maps. Red asterisk and Red-Yellow blobs indicate an increase caused by alcohol consumption. Blue asterisks and blue blobs indicate a reduction. ${ }^{*} p<0.05,{ }^{* *} p<0.01$, $* * * p<0.001$. Data are expressed as mean \pm SEM. 


\subsubsection{Naïve vs Ethanol Exposed SVM Classification}

Next, we evaluated the effect of the overfitting of the model by either including testing set as input of random forest to estimate variable importance or not similar to our previous analysis. Results depicted in Figure 4.20 confirmed the overestimation produced by the exclusion of feature selection step out of the inner loop in the cross validation scheme. Results reproduce the results showed in the previous section confirming optimistic classification performance due to suboptimal analytical design.
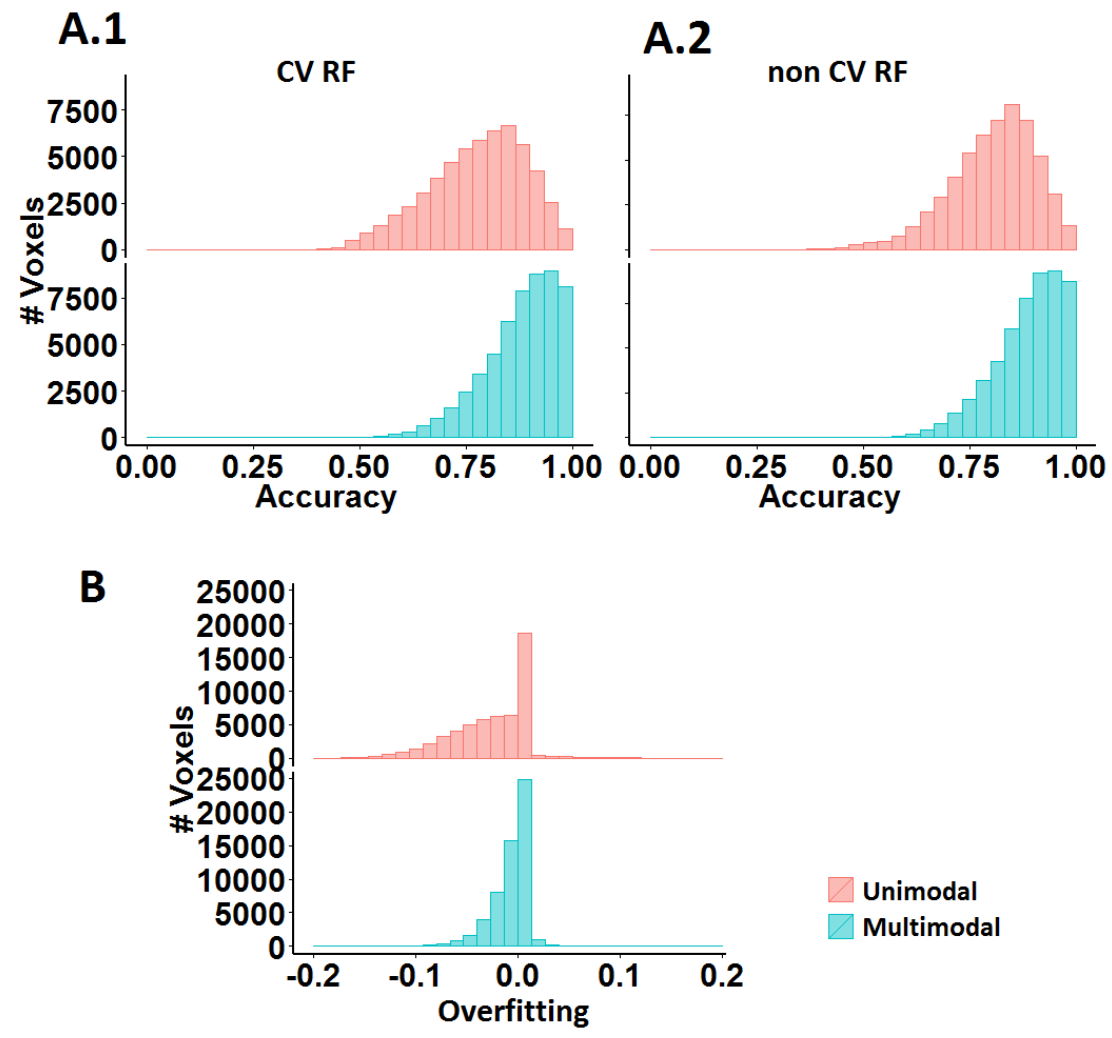

Figure 4.20 Effect of overfitting in voxelwise classification. (A) Overfitting produced by estimation of RF variable importance out the cross-validation loop (A.1) or within the cross-validation loops (A.2). (B) Histogram of the overestimation.

Having minimized the overfitting by inclusion of feature ranking in inner loops of the classification pipeline (see section above), we trained the voxel-wise classifiers to distinguish multimodal structural, diffusion and relaxometry MRI parameters between naïve and alcohol exposed rats. Figure 4.21 summarized brain areas with significant cross-validated classification 
accuracies. Figure 4.21A shows the spatial maps of accuracies for unimodal SVM classifiers (redyellow) and multimodal SVM (blue-green). As can be observed in Figure 4.21B, the combination of multimodal features produce a shift in the voxel wise accuracy towards higher values demonstrating an enhancement in discrimination power from the complementary nature of the multimodal MRI fingerprints. This fact not only represents an increase in the classifier performance, it also manifests that the discrimination power benefits from the complementary nature of the multimodal MRI fingerprints. Multiparametric SVM increases in average a 3.4\% the accuracy (unimodal classifier showed an average accuracy of 0.897 and multimodal classifiers 0.931 ). The benefit is not only present in the overall enhanced model accuracy (Figure $4.21 \mathrm{C}$ left), but also it enhances the sensitivity in the identification of multimodal MRI brain network changes and expands the high classified areas (Figure 4.21C right). The most interesting result is the strong and widespread microstructural reorganization after one month of heavy ethanol drinking allowing almost the perfect discrimination of experimental conditions. 
A

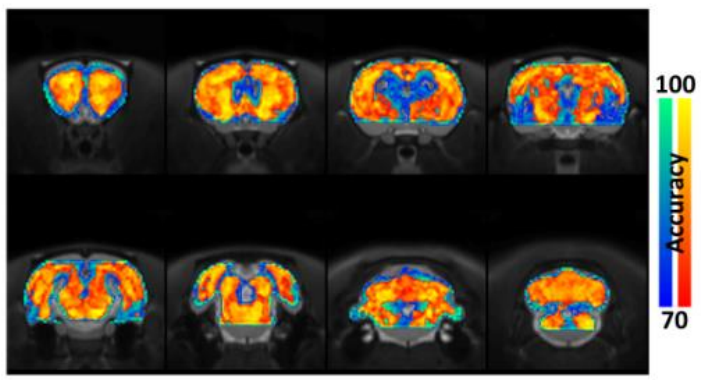

\section{B}
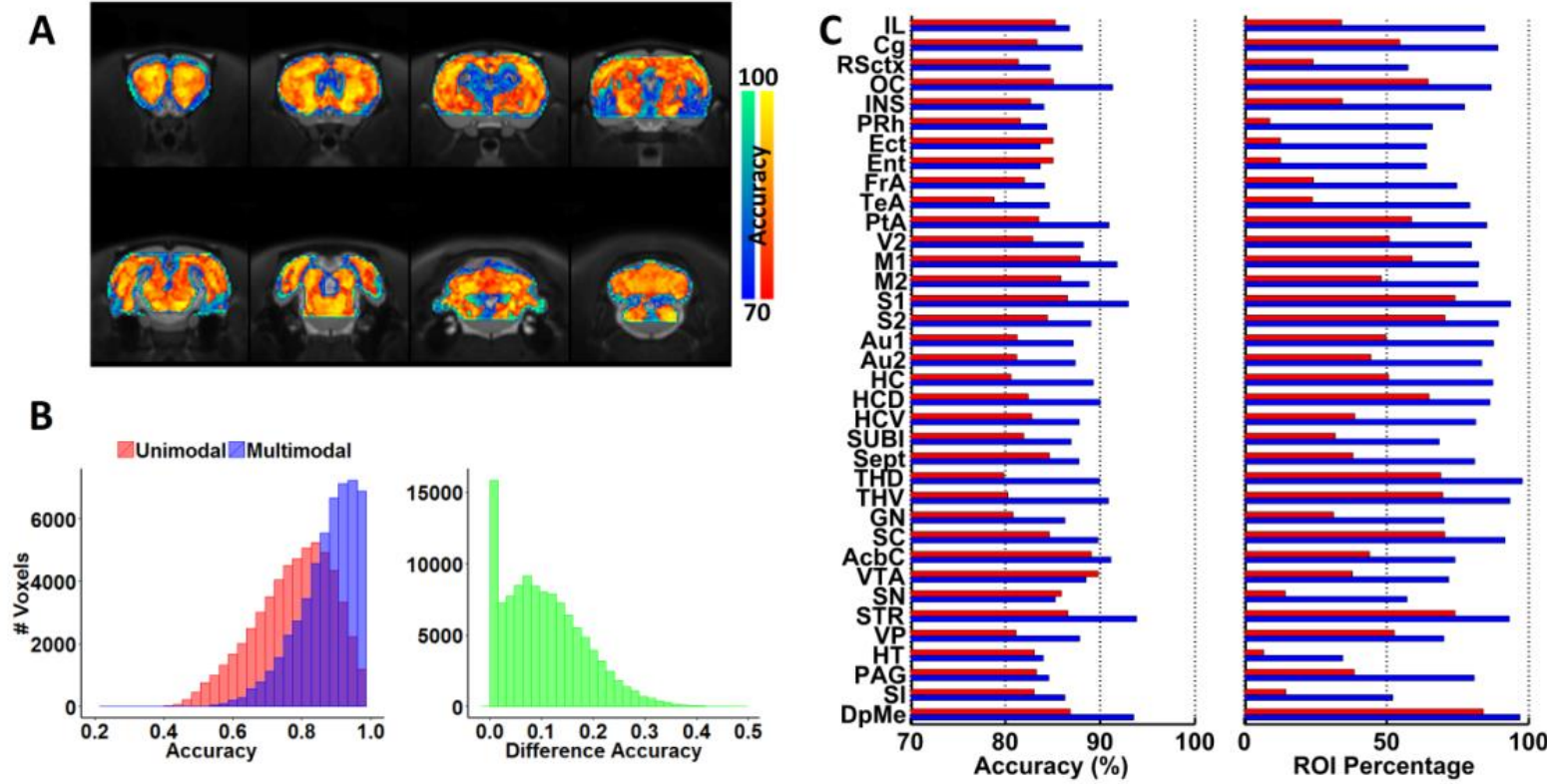

Figure 4.21 Comparison between unimodal and multimodal performance. (A) Voxel wise accuracy maps after multiple comparison correction across space $\left(p<10^{-3}, p_{\text {cluster }}<0.05\right)$ of the SVM model using only the most important modality (blue) and accuracy of the SVM model after selecting those modalities which contribute positively to identify exposed animals (red-yellow). (B) Histogram of intrabrain accuracies to demonstrate that the combination of different modalities shifts the accuracy distribution enhancing discriminatory power (left) and the difference distribution (right). (C) Multimodal voxel-wise classifiers do not just show an increase in accuracy (left), they also allow the identification of other significantly different regions (right).

\section{Is alcohol preference related to SVM prediction probability?}

We were also interested in testing and identifying regional MRI-derived structure or IMSDs that might anticipate the alcohol preference. We decided to use preference ratio instead of the absolute alcohol intake volumes because the water consumption was reduced after 5 days of alcohol availability (136). By using Pearson's correlation for testing relationship between probability of being classified as exposed subjects at Naïve state, we found significant positive correlation ( $p<0.001$ uncorrected) in clusters almost bilaterally localized (19 voxels both clusters) in areas encompassing basal ganglia and amydalar formations $(r=0.804, p<0.001)$. In other words, subjects with higher probability of being classified as exposed at naïve state will show higher alcohol preference in early ethanol exposition. Results are shown in Figure 4.22. 

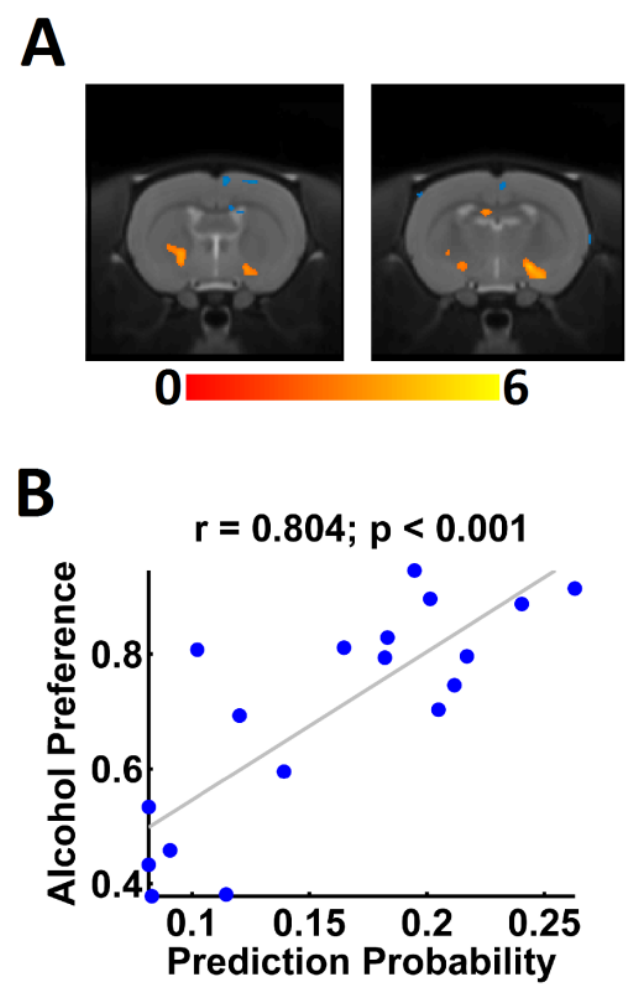

Figure 4.22 Innate evidences of alcohol preference identified by multimodal neuroimaging. (A) Statistical map of significant positive correlation in the basal ganglia. (B) Scatter plot of the average probability of being classified as exposed subject vs the average alcohol preference within days 1-3 of two bottles free choice paradigm.

We also tested the relationship between the initial or final alcohol preference and the exposed state prediction after alcohol consumption (Figure 4.23). While no significant correlations were found after cluster correction for Type I error for the final preference, widespread positive significant correlations were found for the initial alcohol preference $\left(p<0.01, p_{\text {cluster }}<0.05\right)$. 
A

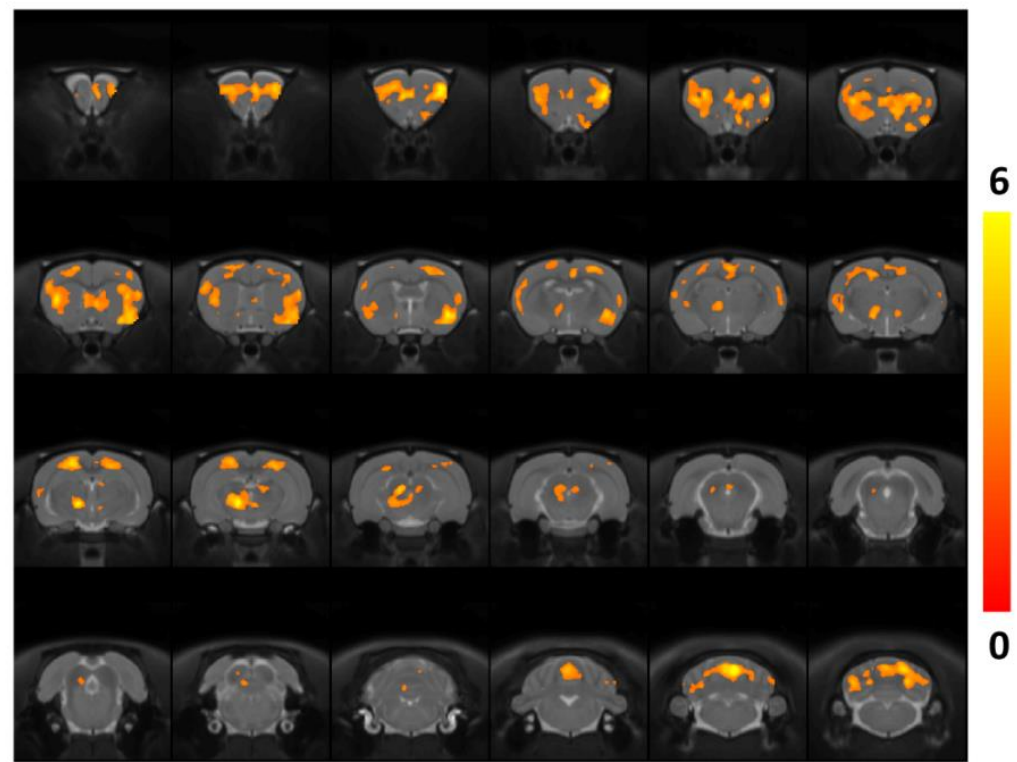

B
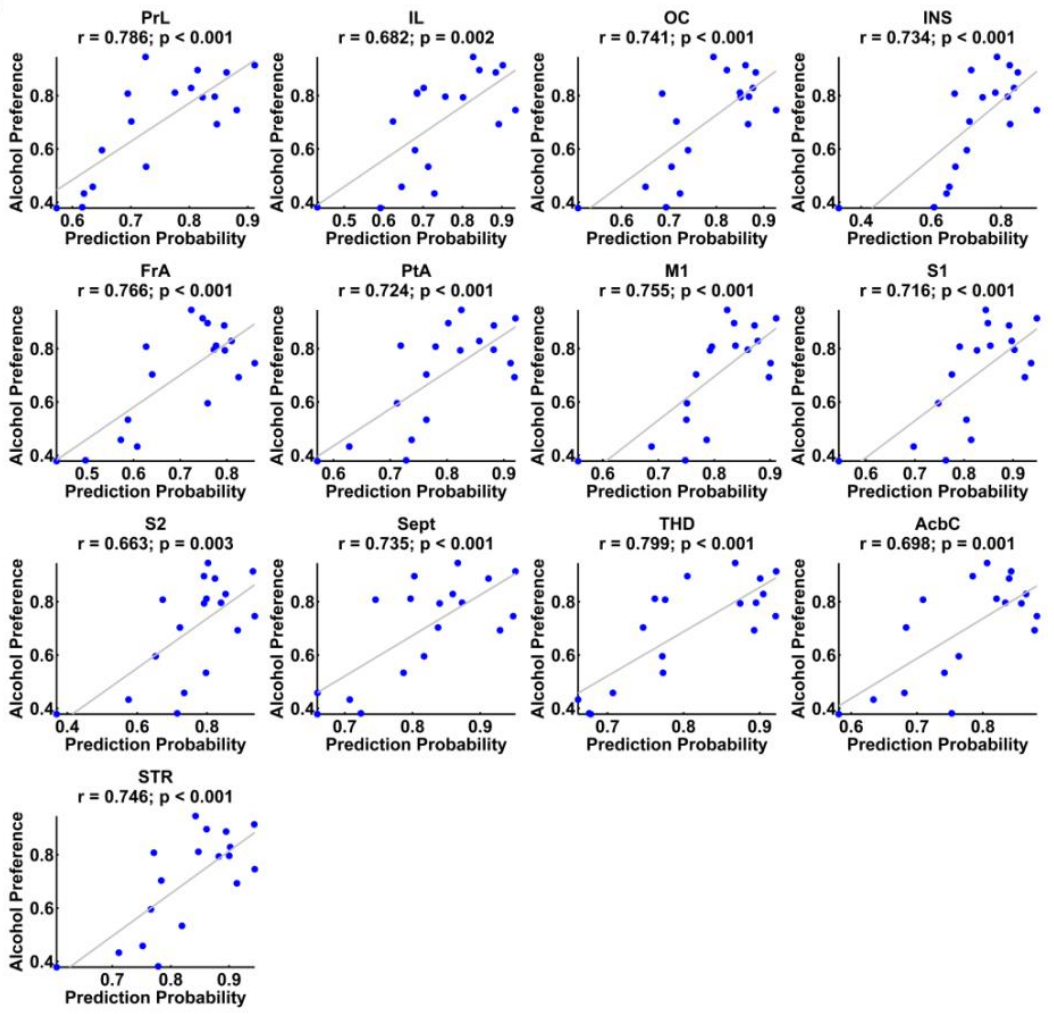

Figure 4.23 Probability of being classified as exposed animals strongly depends on initial alcohol preference but not on later preference. 


\subsubsection{Evaluation of treatment efficacy by SVM}

Having demonstrated that a single voxel provides enough information to classify accurately between Naïve and exposed brain tissue states, the next logical step was to use unbiased classification probability to predict the efficiency of Naltrexone treatment. After confirming that no differences in prediction probabilities at naïve and exposed state between the two subsets exist, voxel-wise two-sample t-test was performed. Our results showed no significant differences in prediction probabilities between modifications of predictions induced by treatment and abstinence. Despite the lack of statistical significancy, we observed some overlapping effects in accumbens and medial brain areas between reduction in probability of being predicted as exposed animals between Naltrexone treated and non-treated animals. Interestingly, strong decreased probability of being classified as exposed animals in cortical areas was only observed in Naltrexone treated animals. Further analysis need to be performed in order to confirm homeostatic changes.
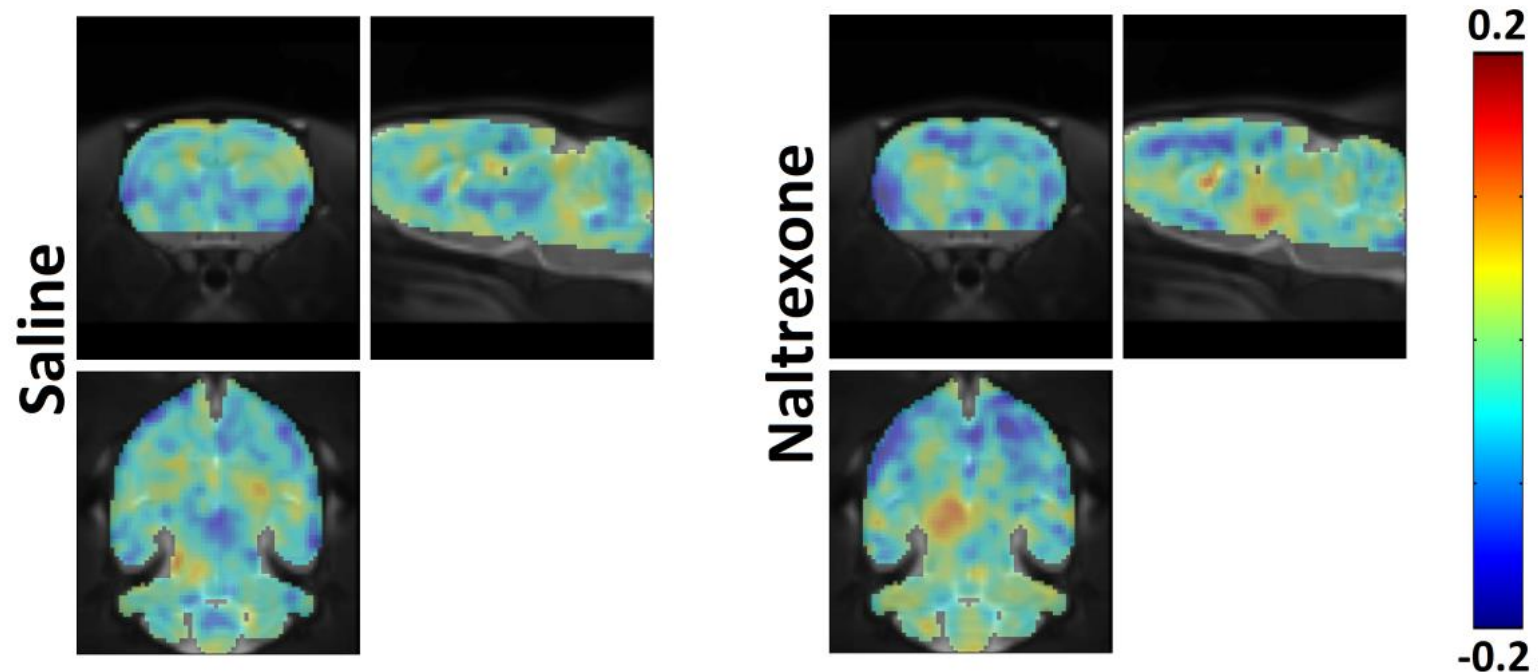

Figure 4.24 Effect of Saline (right) and Naltrexone treatment (left) on the reduction of the prediction of the brain tissue through local multimodal fingerprints. Colorbar represent differences in predictions after saline or naltrexone treatment respect to exposed state. Negative values (blue) represent higher shifting towards Naïve state.

We attempted to put together behavioral aspects represented by ethanol preference and the changes of multimodal fingerprint after treatment estimated as the difference of prediction 
probabilities before and after treatment. Interestingly, significant negative correlation between initial alcohol preference and modifications in thalamic IMSD were found (punc $<0.005, p c<0.06$, Figure 4.25). No significant correlation was found with final preference and Naltrexone effect. Bringing together these results with those previously reported, naltrexone efficacy could be also related to innate aspects which anticipates how much ethanol will be intaked. A non-answered question is whether evidences of causality of modified IMSDs and treatment outcomes exist.
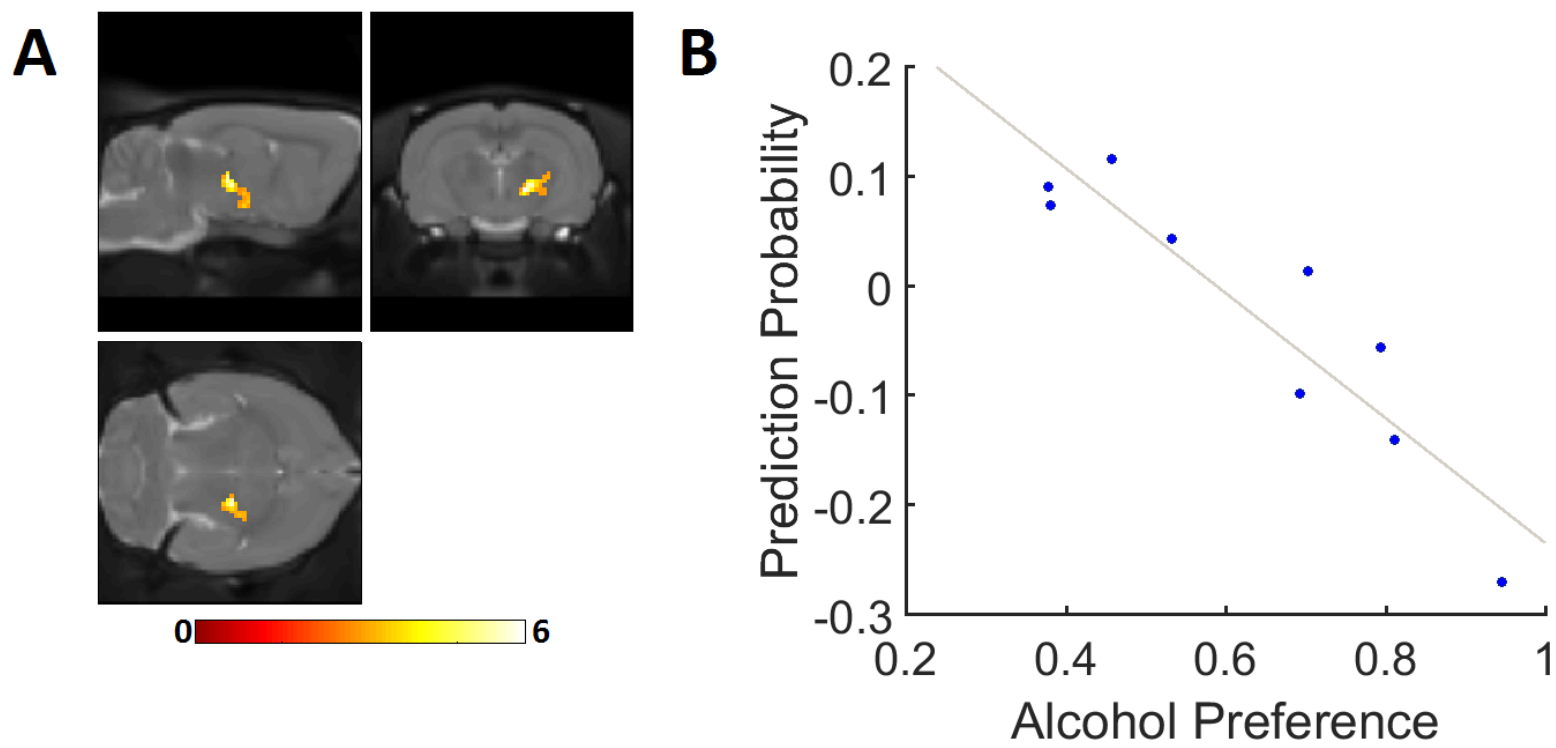

Figure 4.25 Naltrexone efficacy is correlated to initial alcohol preference in the ventral thalamus but not to steady preference. (A) Identified cluster of significant negative. (B) Scatter plot of the changes in IMSDs and initial alcohol preferences. Changes in IMSDs are calculated as difference between exposed prediction after and before the treatment. More negative values mean higher effects.

\subsubsection{Discussion}

In this section we have confirm the robust classification power provided by combination of multimodal MRI. This approach differs substantially from the approach proposed in the previous sections in the way that features are extracted: mROI-based above and voxel-wise in the current section. As well, the proposed classification approach aims to make predictions a single voxel level allowing to differentiate within-subject heterogeneous responses. This is an important aspect in the study of psychiatric diseases often multidimensional and characterized by multiple comorbidities. 
As expected, classification accuracies were enhanced by multimodal combination of features due to the complementary neurobiological information contained in different MRI parameters. However, unimodal MSMCA classifier provides accurate predictive power. This is not surprising given the profound changes occurring in the brain tissue during a month of heavy alcohol consumption also identified in the previous analysis. By univariate analysis, we identified increased $\mathrm{MD}, \mathrm{GMv}$, and $\mathrm{WMv}$ and decreased $\mathrm{T} 2$ relaxation times widely spread across different brain regions. Minor changes in FA and T1 were found. The observed severe brain changes might be a priori partly explained by normal aging. However, when compared to control msP rats, alcohol exposed msP rats showed different brain maturation. Our results were mostly consistent with those results published by others. Inconsistent findings have been reported how brain diffusivity evolves with the age. Previous studies have shown that brain myelination ends by the end of the third postnatal week (137) with a slow progressive decrease in FA values with MD approximately stable from postnatal day 80 (138). Other authors, in contrast, have reported declined MD in the brain with no changes in FA and consistent brain enlargement (139). Of note, aforementioned studies carried out ex vivo MRI in fixed brains which has been demonstrated to alter brain diffusivity (140). Consistent T2 declination has been reported (141). Despite these considerations, it is undeniable, however, that the alcohol is the most contributable factor to discriminate both timepoints. This is confirmed by our correlation analysis where surprisingly classification-derived posterior probabilities advance some alcohol-related behaviors.

We investigated the relation of IMSDs with alcohol-related behaviors, specifically with alcohol preference. We found significant correlation in the basal ganglia, particularly in voxels encompassing basal ganglia and some amydalar nucleus with initial alcohol preferences. In light of these results, in addition to other unpredictable reasons which trigger higher alcohol consumption, an innate anatomical predisposition seems to exist. However, biological mechanisms underlying such microstructure environment promoting higher alcohol preference are hardly identifiable from MRI parametric measures. One of plausible scenarios is the role of limbic system in begin and maintenance of alcohol-related mental disorders. Dopaminergic function in the basal ganglia plays a key role in the modulatory effects of motivation and response to hedonic reward (142). Reward, pleasure, or hedonism increases the probability of occurrence of a particular behavior such as addiction. This phenomenon is known as positive reinforcement and is pivotal in the acquisition of 
addictive behaviors. In contrast to pleasing circumstances produced by alcohol consumption, other implications of disrupted microstructure in the limbic system might be related to modulation of negative reinforcement produced during withdrawal episodes or other negative sensations. Effects found in the amygdalar nucleus to its role in anxiety and fear circuits as fundamental for acquisition of alcohol-related disorders $(143,144)$. Previous studies proposed that msP rats showing innate high levels of anxiety and depression-like behaviors alleviate negative states by alcohol selfadministration (145).

Our analysis also found widespread significant correlations of initial alcohol preference and the IMSD's immediately after restricting alcohol access but no relationship with later alcohol preference. Stated in another way, early alcohol drinking pattern might define the addiction development and the MRI tissue characterization. Significant microstructural dependence on early alcohol preference and absence in later experimental stages evidences possible neuroadaptations occurring during the first days of alcohol access. Harrington and coworkers identified eight different alcohol intake profiles which might explain heterogeneous response to treatment (12). Our findings manifest the importance of designing clinical interventions based on lifespan intake patterns to enhance treatment efficacies.

IMSDs were not significantly different under the two abstinence conditions, however antirelapse Naltrexone treatment might modify divergently the evolution of brain microstructure (77). It is important to point that medication seems to "repair" brain tissue in some cortical areas at the cost of altering hypothalamic fingerprint. This might explain the sleepiness as a common sideeffect associated to Naltrexone treatment. However, although promising, the homeostatic effect of Naltrexone treatment in relapse needs to be further confirmed.

Finally, we aimed to identify the relationship between unbiased measures of brain recovery by quantification of IMSDs and the pattern of drinking. Recovery from drug dependence and treatment efficacies are often complex and multidimensional phenomenon depending on a wide range of behavioral or genetic factors (146). While naltrexone treatment is related to changes in outcomes related to the drinking (147-149), little is known about reciprocal interactions, namely how drinking influences treatment. It is well-known that the proclivity to alcohol drinking by rodents depends in part in its sweet flavor. A recent work has suggested a relationship between 
sweet-liking phenotype and Naltrexone response in a randomized clinical Trial (150). In line with the human literature, our results show higher Naltrexone effect in the ventral thalamic IMSD when a higher initial preference for $10 \%$ Ethanol exists. It is noteworthy that ventral thalamic nucleus is involved in processing reward-relevant information by GABAergic inputs from VTA/SN which could modulate alcohol preference and naltrexone efficacy (151).

In summary, in this section, we have investigated the use of voxel-wise classification models to differentiate Naïve from alcohol exposed msPs rats by multimodal brain imaging. We found high local prediction accuracies spanning nearly the entire brain and surpassing single modality predictions. These results confirm the results obtained in the previous section (4.2) whereas less spatial-specific fingerprints assigned correctly the $100 \%$ of the samples. We furthermore have explored the relationship with behavioral aspects of the msPs rats with brain imaging. Specifically, a strong relationship between multimodal MRI-derived biomarkers with initial alcohol preference has been found prior to alcohol access suggesting some neural characteristics underlying innate alcohol preference. Interestingly, in line with recent human studies, a negative correlation between alcoholliking behavior and Naltrexone efficacy has been evidenced. Having probed the use of machine learning tools to differentiate individual-wise alcohol-related conditions, in the current section we have demonstrated that multimodal and multivariate classification techniques are also powerful tools to investigate mesoscopic disease development and evaluate treatment efficacies. 
Multimodal MRI to identify pathological states in msP rats 


\section{General discussion}

Having demonstrated the use of multimodal signature to discriminate different experimental conditions in $\mathrm{msP}$ rats, in the current investigation we develop and evaluate a methodological approach to increase the classification accuracy combining multimodal neuroimaging data as an alternative to traditional statistical analysis where type I errors must be controlled, and we have gone one step further using the implemented model to predict the progression of the brain alterations produced by the alcohol consumption. As far as we know, no works uses multimodal voxel-wise information to learn predictive models using machine learning procedures. Specifically, utilizing combinations of data from structural imaging relaxometry MRI maps, DTI modalities and algorithms derived from the machine learning such as Support Vector Machine classification and feature selection provide excellent results. The most attractive feature is the remarkable improvement respect to the traditional mass-variate analysis in the sensitivity to detect changes due to de alcohol exposition or even to detect the effect of Naltrexone on the microstructural properties. A direct consequence of this fact is that the addition of many MRI modalities or even combining parametric maps of diverse nature increases the sensibility of detecting anatomical, microstructural, cellular or functional alterations of the neuroimaging studies and the possibility of predicting the disease progression in terms of microstructure and definition of addiction vulnerability or resiliency biomarkers.

The use of machine learning tools in the study of psychiatric diseases has attracted the interest of scientists in the last years making it evolve rapidly. We address here some of the future work that will be surely conducted in order to progress in the better understanding of the brain using the multimodal fusion of in vivo neuroimaging data.

Despite the excellent results provided, prediction analyses are very optimistic when the number of predictors is high relative to the number of samples (152). While cross-validation provides strong validation for the training test, predictability of external datasets could be limited. We found this situation when different experimental conditions in 18 subjects (at best) are classified using 196 or 6 (at worst for first and second approaches presented in this work, respectively). Even though a feature selection approach is part of our framework to reduce the number of predictors in 
the classification model, the sample size should be increased in order to reduce the variance of the prediction and ensure the validity of the results here obtained. An additional validation subset is needed to investigate the reproducibility of our results.

The proposed machine learning approach at a voxel-level assumes that the abstinence (either if the animals are treated with Naltrexone or not) is not a state by itself and can be classified as a baseline state or a post-heavy alcohol drinking state. However, this assumption cannot be fully fulfilled based on our PCA results since the evolution of the brain during the abstinence cannot be longer predicted.

Here we explore the microstructure of the brain using parametric maps such as T1 and T2 relaxometry times which is associated with iron deposition and microglia activation $(153,154)$. In addition, diffusion parameters are also included in our models (FA and MD). As previously reported, others diffusion metrics such as RD or AD are more sensitive in the detection of demyelination or axonal disruption $(155,156)$ and the classification models might be improved integrating additional parametric maps. However, water motion and diffusion processes can be accurately described by its magnitude (MD) and anisotropy (FA) (157). Note that the inclusion of additional parameters would increase the model dimensionality raising the risk of overfitting (99). This fact is not simply restricted to the diffusion measurement, combining other imaging modalities such as fMRI, histology, or even behavioral or clinical measures that complement the model could benefit the performance of the prediction models and, therefore, increases the discrimination power (71).

Despite the promising results, formulated statements here are subjected to strong limitations. The conclusions obtained are subjected to very restringing aspects. Classification models to predict the exposition to alcohol and progression of abstinence are trained using longitudinal information without consideration of the natural evolvement of the tissues. Some alcohol and abstinence effects here exposed do not correct the effect of the age or the acute effect of the EtoH. In order to consider the age confound, age-control group should be included in the multivariate analytical framework. The accumulative effect of the alcohol might produce non-permanent effects in the brain such as cytotoxic or vasogenic edema which could alter our results (40). Measures such as Blood Alcohol Content (BAC) among others can be used as confounds in our statistical models. On the other hand, treatment predictions are based on animals injected with Naltrexone and not 
treated during the withdrawal. All these animals are subjected to the same experimental history; therefore this possible flaw does not exist. Behavioral assessment after Naltrexone treatment is lacking and we cannot confirm that IMSDs also influence later alcohol-related behaviors. Further experiments are needed to identify the causality between MRI parameters and inhibited alcohol seeking behaviors after Naltrexone treatment. Alcohol research could benefit of identifying those neurochemical and cellular mechanisms driving MRI changes along the longitudinal experimental conditions ranking from the alcohol exposition and treated withdrawal. Ultimately, the translational value of the proposed microstructural markers and their clinical utility has to be tested in human populations.

From a neurobiological perspective, brain alterations characterized by MSDs in the global (mROls) or local form (voxel-wise) provide quantitative information about tissue microstructure that may be also used to fill the gap between non-invasive clinical observations and neurobiological mechanisms. As mentioned in the introduction, a number of cellular changes have been correlated with alterations in acquired MRI parameters. For instance, changes in brain MD as predominantly found in our study have been frequently associated to neuroinflammatory processes and cellular necrosis (158). In a recent longitudinal study of Wallerian degeneration contrasting MRI measurements with immunohistochemical evidence in cats (159); increased MD was clearly correlated with microglial activation and astrocytosis. Interestingly, neuroinflammation has been recently proposed as a mechanism of alcohol-related brain damage $(160,161)$. If this were the case, the changes induced in the structure of brain parenchyma by a neuroinflammatory process, affecting the tortuosity and/or volume of the extracellular space, or inducing iron accumulation (34, $40,46,47)$ would help explain some of the individual MRI alterations found in our study. Intriguingly, one postulated action of naltrexone is as anti-inflammatory (162-164), reverting glial activation in the brain (160). In this regard, the specific multimodal signature found in our study for naltrexone treated brains, including a significant recovery of alcohol-induced MD increase, lends support to the neuroinflammatory hypothesis of alcohol-related brain damage and the antiinflammatory effects of naltrexone. Supporting our inflammatory processes hypothesis, relaxometry (longitudinal and transversal) changes relates to the amount of free water and the structure of intracellular water (by means of de- and rehydration mechanisms) and therefore, fluid exchange through neuronal membranes associated to alcohol abuse played a fundamental role in the success 
of the discrimination across different disease stages (165).

Other indirect factors potentially affecting tissue microstructure, as alcohol-triggered alterations in neurotransmission and neuronal physiology in general, with an impact on ionic gradients and energy metabolism, cannot be discarded. In this respect, chronic alcohol consumption by high alcohol preferring rats induces global effects on neuronal activity in many brain regions (166). Importantly, and in line with the present results, changes in activity in that study persisted after one week of abstinence. Although the precise biological changes behind the multimodal MRI alterations found in the present study are yet not known, the obtained results assure further and intensive research in that direction. 


\section{Conclusions}

This thesis deals with the study of acquisition and maintenance of addictive-behaviors in msP rats, genetically selected rats showing high voluntary alcohol preference, by multimodal MR neuroimaging. To take benefit from the complementary nature of different MRI modalities, we have developed a machine-learning based approach to make subject-wise predictions. Based on this framework, two evaluations have been performed. First, a set of biomarkers derived from datadriven brain parcellation is evaluated. Second, in order to advance in the understanding of brain adaptations in acquisition and treatment alcohol-related behaviors, predictive models at mesoscopic scales were trained and used to evaluate treatment effects.

In the following, the main findings obtained throughout this research are summarized:

- Neuroimaging biomarkers are useful to discriminate several alcohol-related conditions in msP rats. Our data-driven parcellation and biomarkers definition allowed us classifying conditions with different levels of accuracy.

- Predictive models multimodal neuroimaging biomarkers overperform those trained with a single modality-derived biomarkers.

- Suboptimal design of analytical frameworks could lead overestimation in the discriminative power.

- Single-voxel based classifiers identify accurately one month of heavy alcohol consumption in msP rats.

- Prediction probabilities are associated with alcohol-seeking behaviors in basal ganglia and amygdalar formation, two well-known brain areas related to addiction.

- Naltrexone treatment might cause homeostatic brain adaptations during abstinence related to seeking behaviors.

To conclude, with technological advances in medicine and health care systems, psychiatric diseases can be characterized by increasingly diverse and complex data sets derived from neuroimaging, demographic or clinical information. Despite the health benefits for the society, the integration of multisource information in computational models is challenging and we are far away from using automatic diagnosis tools based on neuroimaging datasets. However, the investigations around this topic are encouraging. Along this dissertation, we demonstrate here the fusion of multimodal MRI data sets benefits the study of mental diseases and particularly in the study of the alcohol disorders and its treatment with Naltrexone during the abstinence. 
Multimodal MRI to identify pathological states in msP rats 


\section{References}

1. WHO Regional Office for Europe. Status report on alcohol and health in 35 European countries 2013. Other. Copenhagen: WHO Regional Office for Europe; 2013.

2. World Health Organization. Global status report on alcohol and health 2014. Other. Geneva: World Health Organization; 2014.

3. Rehm JU, Shield KD, Rehm MX, Gmel G, Frick U. Alcohol consumption, alcohol dependence and attributable burden of disease in Europe. Potential gains from effective interventions for alcohol dependenceToronto: Centre for Addiction and Mental Health. 2012.

4. Corrao G, Bagnardi V, Zambon A, La Vecchia C. A meta-analysis of alcohol consumption and the risk of 15 diseases. Preventive Medicine. 2004;38(5):613-9.

5. Babor T, World Health Organization. Department of Mental H, Substance D. AUDIT, the Alcohol Use Disorders Identification Test: Guidelines for Use in Primary Care: World Health Organization, Department of Mental Health and Substance Dependence; 2001.

6. Koob GF, Buck CL, Cohen A, Edwards S, Park PE, Schlosburg JE, et al. Addiction as a stress surfeit disorder. Neuropharmacology. 2014;76 Pt B:370-82.

7. American Psychiatric A, American Psychiatric A, Force DSMT. Diagnostic and statistical manual of mental disorders : DSM-5. 2013.

8. Sommer WH, Spanagel R. Behavioral neurobiology of alcohol addiction: Springer; 2013.

9. Bell RL, Hauser S, Rodd ZA, Liang T, Sari Y, McClintick J, et al. A Genetic Animal Model of Alcoholism for Screening Medications to Treat Addiction. Int Rev Neurobiol. 2016;126:179-261.

10. Johnson BA. Medication treatment of different types of alcoholism. Am J Psychiatry. 2010;167(6):630-9.

11. Leggio L, Kenna GA, Fenton M, Bonenfant E, Swift RM. Typologies of alcohol dependence. From Jellinek to genetics and beyond. Neuropsychol Rev. 2009;19(1):115-29.

12. Harrington M, Velicer WF, Ramsey S. Typology of alcohol users based on longitudinal patterns of drinking. Addict Behav. 2014;39(3):607-21.

13. Verhulst B, Neale MC, Kendler KS. The heritability of alcohol use disorders: a meta-analysis of twin and adoption studies. Psychol Med. 2015;45(5):1061-72.

14. Slutske WS, True WR, Scherrer JF, Heath AC, Bucholz KK, Eisen SA, et al. The heritability of alcoholism symptoms: "indicators of genetic and environmental influence in alcohol-dependent individuals" revisited. Alcohol Clin Exp Res. 1999;23(5):759-69. 
15. Bell RL, Rodd ZA, Engleman EA, Toalston JE, McBride WJ. Scheduled access alcohol drinking by alcohol-preferring $(P)$ and high-alcohol-drinking (HAD) rats: modeling adolescent and adult bingelike drinking. Alcohol. 2014;48(3):225-34.

16. Ciccocioppo R, Economidou D, Cippitelli A, Cucculelli M, Ubaldi M, Soverchia L, et al. Genetically selected Marchigian Sardinian alcohol-preferring (msP) rats: an animal model to study the neurobiology of alcoholism. Addict Biol. 2006;11(3-4):339-55.

17. Stopponi S, de Guglielmo G, Somaini L, Cippitelli A, Cannella N, Kallupi M, et al. Activation of PPARgamma by pioglitazone potentiates the effects of naltrexone on alcohol drinking and relapse in msP rats. Alcohol Clin Exp Res. 2013;37(8):1351-60.

18. Yardley MM, Ray LA. Medications development for the treatment of alcohol use disorder: insights into the predictive value of animal and human laboratory models. Addict Biol. 2017;22(3):581-615.

19. Boksa P. A way forward for research on biomarkers for psychiatric disorders. J Psychiatry Neurosci. 2013;38(2):75-7.

20. Neufeldt V, Sparks AN. Webster's new world dictionary: Simon and Schuster; 2002.

21. Bühler $M$, Mann K. Alcohol and the Human Brain: A Systematic Review of Different Neuroimaging Methods. Alcoholism: Clinical and Experimental Research. 2011;35(10):1771-93.

22. Demirakca T, Ende G, Kämmerer N, Welzel-Marquez H, Hermann D, Heinz A, et al. Effects of alcoholism and continued abstinence on brain volumes in both genders. Alcoholism: Clinical and Experimental Research. 2011;35(9):1678-85.

23. Rando K, Hong KI, Bhagwagar Z, Li CS, Bergquist K, Guarnaccia J, et al. Association of frontal and posterior cortical gray matter volume with time to alcohol relapse: a prospective study. The American Journal of Psychiatry. 2011;168(2):183-92.

24. Fein G, Landman B, Tran H, McGillivray S, Finn P, Barakos J, et al. Brain atrophy in long-term abstinent alcoholics who demonstrate impairment on a simulated gambling task. Neurolmage. 2006;32(3):1465-71.

25. Makris N, Oscar-Berman M, Jaffin SK, Hodge SM, Kennedy DN, Caviness VS, et al. Decreased volume of the brain reward system in alcoholism. Biological psychiatry. 2008;64(3):192-202.

26. Kril JJ, Halliday GM, Svoboda MD, Cartwright $\mathrm{H}$. The cerebral cortex is damaged in chronic alcoholics. Neuroscience. 1997;79(4):983-98.

27. Gazdzinski S, Durazzo TC, Yeh PH, Hardin D, Banys P, Meyerhoff DJ. Chronic cigarette smoking modulates injury and short-term recovery of the medial temporal lobe in alcoholics. Psychiatry research. 2008;162(2):133-45. 
28. Pfefferbaum A, Sullivan EV, Rosenbloom MJ, Mathalon DH, Lim KO. A controlled study of cortical gray matter and ventricular changes in alcoholic men over a 5-year interval. Archives of General Psychiatry. 1998;55(10):905-12.

29. Hommer DW, Momenan R, Kaiser E, Rawlings RR. Evidence for a Gender-Related Effect of Alcoholism on Brain Volumes. American Journal of Psychiatry. 2001;158(2):198-204.

30. Pfefferbaum A, Rosenbloom M, Deshmukh A, Sullivan E. Sex differences in the effects of alcohol on brain structure. The American Journal of Psychiatry. 2001;158(2):188-97.

31. Mann K. Neuroimaging of gender differences in alcohol dependence: are women more vulnerable? Alcoholism: clinical and experimental research. 2005;29:896-901.

32. Pfefferbaum A. In vivo detection and functional correlates of white matter microstructural disruption in chronic alcoholism. Alcoholism: clinical and experimental research. 2000;24:1214-21.

33. Pfefferbaum A. Degradation of association and projection white matter systems in alcoholism detected with quantitative fiber tracking. Biological psychiatry. 2009;65:680-90.

34. Pfefferbaum A, Sullivan EV. Disruption of brain white matter microstructure by excessive intracellular and extracellular fluid in alcoholism: evidence from diffusion tensor imaging. Neuropsychopharmacology : official publication of the American College of Neuropsychopharmacology. 2005;30(2):423-32.

35. Chanraud S, Reynaud M, Wessa M, Penttila J, Kostogianni N, Cachia A, et al. Diffusion tensor tractography in mesencephalic bundles: relation to mental flexibility in detoxified alcoholdependent subjects. Neuropsychopharmacology : official publication of the American College of Neuropsychopharmacology. 2009;34(5):1223-32.

36. Yeh PH, Simpson K, Durazzo TC, Gazdzinski S, Meyerhoff DJ. Tract-Based Spatial Statistics (TBSS) of diffusion tensor imaging data in alcohol dependence: abnormalities of the motivational neurocircuitry. Psychiatry research. 2009;173(1):22-30.

37. Pfefferbaum A, Adalsteinsson E, Sullivan EV. Supratentorial profile of white matter microstructural integrity in recovering alcoholic men and women. Biological psychiatry. 2006;59(4):364-72.

38. Pfefferbaum A, Sullivan EV. Microstructural but not macrostructural disruption of white matter in women with chronic alcoholism. Neurolmage. 2002;15(3):708-18.

39. Pfefferbaum A, Lim KO, Zipursky RB, Mathalon DH, Rosenbloom MJ, Lane B, et al. Brain gray and white matter volume loss accelerates with aging in chronic alcoholics: a quantitative MRI study. Alcoholism, Clinical and Experimental Research. 1992;16(6):1078-89.

40. Kong LM, Zheng WB, Lian GP, Zhang HD. Acute effects of alcohol on the human brain: Diffusion tensor imaging study. American Journal of Neuroradiology. 2012;33(5):928-34. 
41. Koenig SH, Brown RD, 3rd, Spiller M, Lundbom N. Relaxometry of brain: why white matter appears bright in MRI. Magnetic resonance in medicine : official journal of the Society of Magnetic Resonance in Medicine / Society of Magnetic Resonance in Medicine. 1990;14(3):482-95.

42. MacKay A, Whittall K, Adler J, Li D, Paty D, Graeb D. In vivo visualization of myelin water in brain by magnetic resonance. Magnetic resonance in medicine : official journal of the Society of Magnetic Resonance in Medicine / Society of Magnetic Resonance in Medicine. 1994;31(6):673-7.

43. Sullivan EV, Pfefferbaum A. Magnetic resonance relaxometry reveals central pontine abnormalities in clinically asymptomatic alcoholic men. Alcoholism, Clinical and Experimental Research. 2001;25(8):1206-12.

44. Sheu YS, Polcari A, Anderson CM, Teicher MH. Harsh corporal punishment is associated with increased T2 relaxation time in dopamine-rich regions. Neurolmage. 2010;53(2):412-9.

45. Bell-Temin H, Zhang P, Chaput D, King MA, You M, Liu B, et al. Quantitative proteomic characterization of ethanol-responsive pathways in rat microglial cells. Journal of proteome research. 2013;12(5):2067-77.

46. Dennis CV, Sheahan PJ, Graeber MB, Sheedy DL, Kril JJ, Sutherland GT. Microglial proliferation in the brain of chronic alcoholics with hepatic encephalopathy. Metabolic brain disease. 2013.

47. McClain JA, Morris SA, Deeny MA, Marshall SA, Hayes DM, Kiser ZM, et al. Adolescent binge alcohol exposure induces long-lasting partial activation of microglia. Brain, behavior, and immunity. 2011;25 Suppl 1:S120-8.

48. Brass SD, Chen NK, Mulkern RV, Bakshi R. Magnetic resonance imaging of iron deposition in neurological disorders. Top Magn Reson Imaging. 17. United States2006. p. 31-40.

49. Gozzi A, Agosta F, Massi M, Ciccocioppo R, Bifone A. Reduced limbic metabolism and frontocortical volume in rats vulnerable to alcohol addiction. Neurolmage. 2013;69(0):112-9.

50. Ashburner J, Friston KJ. Voxel-based morphometry--the methods. Neuroimage. 2000;11(6 Pt 1):805-21.

51. Lipton ML, Gellella E, Lo C, Gold T, Ardekani BA, Shifteh K, et al. Multifocal white matter ultrastructural abnormalities in mild traumatic brain injury with cognitive disability: a voxel-wise analysis of diffusion tensor imaging. Journal of neurotrauma. 2008;25(11):1335-42.

52. Qiu D, Tan L-H, Zhou K, Khong P-L. Diffusion tensor imaging of normal white matter maturation from late childhood to young adulthood: voxel-wise evaluation of mean diffusivity, fractional anisotropy, radial and axial diffusivities, and correlation with reading development. Neuroimage. 2008;41(2):223-32.

53. Mosconi L, Tsui W-H, De Santi S, Li J, Rusinek H, Convit A, et al. Reduced hippocampal metabolism in $\mathrm{MCl}$ and AD Automated FDG-PET image analysis. Neurology. 2005;64(11):1860-7. 
54. Jeong $\mathrm{Y}$, Cho SS, Park JM, Kang SJ, Lee JS, Kang E, et al. 18F-FDG PET findings in frontotemporal dementia: an SPM analysis of 29 patients. J Nucl Med. 2005;46(2):233-9.

55. Zang $Y-F$, He $Y$, Zhu C-Z, Cao Q-J, Sui $M-Q$, Liang $M$, et al. Altered baseline brain activity in children with ADHD revealed by resting-state functional MRI. Brain \& development. 2007;29(2):8391.

56. Wang Z, Li Y, Childress AR, Detre JA. Brain entropy mapping using fMRI. Plos one. 2014;9(3):e89948.

57. Kelly C, Zuo XN, Gotimer K, Cox CL, Lynch L, Brock D, et al. Reduced interhemispheric resting state functional connectivity in cocaine addiction. Biol Psychiatry. 2011;69(7):684-92.

58. Fjell AM, Walhovd KB, Fennema-Notestine C, McEvoy LK, Hagler DJ, Holland D, et al. CSF biomarkers in prediction of cerebral and clinical change in mild cognitive impairment and Alzheimer's disease. J Neurosci. 2010;30(6):2088-101.

59. Norman KA, Polyn SM, Detre GJ, Haxby JV. Beyond mind-reading: multi-voxel pattern analysis of fMRI data. Trends Cogn Sci. 2006;10(9):424-30.

60. Mahmoudi A, Takerkart S, Regragui F, Boussaoud D, Brovelli A. Multivoxel pattern analysis for FMRI data: a review. Comput Math Methods Med. 2012;2012:961257.

61. Zhu X, Suk HI, Shen D. Multi-modality canonical feature selection for Alzheimer's disease diagnosis. Med Image Comput Comput Assist Interv. 2014;17(Pt 2):162-9.

62. Lebedev AV, Westman E, Van Westen GJ, Kramberger MG, Lundervold A, Aarsland D, et al. Random Forest ensembles for detection and prediction of Alzheimer's disease with a good betweencohort robustness. Neuroimage Clin. 2014;6:115-25.

63. Klöppel S, Stonnington CM, Chu C, Draganski B, Scahill RI, Rohrer JD, et al. Automatic classification of MR scans in Alzheimer's disease. Brain. 2008;131(3):681-9.

64. lannaccone R, Hauser TU, Ball J, Brandeis D, Walitza S, Brem S. Classifying adolescent attention-deficit/hyperactivity disorder (ADHD) based on functional and structural imaging. Eur Child Adolesc Psychiatry. 2015;24(10):1279-89.

65. Wang $\mathrm{X}$, Jiao $\mathrm{Y}$, Tang $\mathrm{T}$, Wang $\mathrm{H}$, Lu Z. Altered regional homogeneity patterns in adults with attention-deficit hyperactivity disorder. Eur J Radiol. 2013;82(9):1552-7.

66. Cantor-Rivera D, Khan AR, Goubran M, Mirsattari SM, Peters TM. Detection of temporal lobe epilepsy using support vector machines in multi-parametric quantitative MR imaging. Comput Med Imaging Graph. 2015;41:14-28.

67. Munsell BC, Wee CY, Keller SS, Weber B, Elger C, da Silva LA, et al. Evaluation of machine learning algorithms for treatment outcome prediction in patients with epilepsy based on structural connectome data. Neuroimage. 2015;118:219-30. 
68. Kloppel S, Stonnington CM, Barnes J, Chen F, Chu C, Good CD, et al. Accuracy of dementia diagnosis: a direct comparison between radiologists and a computerized method. Brain. 2008;131(Pt 11):2969-74.

69. Luo SX, Martinez D, Carpenter KM, Slifstein M, Nunes EV. Multimodal predictive modeling of individual treatment outcome in cocaine dependence with combined neuroimaging and behavioral predictors. Drug Alcohol Depend. 2014;143:29-35.

70. Squeglia LM, Ball TM, Jacobus J, Brumback T, McKenna BS, Nguyen-Louie TT, et al. Neural predictors of initiating alcohol use during adolescence. American journal of psychiatry. 2016:appi. ajp. 2016.15121587.

71. Whelan R, Watts R, Orr CA, Althoff RR, Artiges E, Banaschewski T, et al. Neuropsychosocial profiles of current and future adolescent alcohol misusers. Nature. 2014;512(7513):185-9.

72. Ito JR, Donovan DM. Predicting drinking outcome: demography, chronicity, coping, and aftercare. Addict Behav. 1990;15(6):553-9.

73. Seo S, Mohr J, Beck A, Wustenberg T, Heinz A, Obermayer K. Predicting the future relapse of alcohol-dependent patients from structural and functional brain images. Addict Biol. 2015;20(6):1042-55.

74. Morris LS, Kundu P, Baek K, Irvine MA, Mechelmans DJ, Wood J, et al. Jumping the Gun: Mapping Neural Correlates of Waiting Impulsivity and Relevance Across Alcohol Misuse. Biol Psychiatry. 2016;79(6):499-507.

75. Puls I, Mohr J, Wrase J, Priller J, Behr J, Kitzrow W, et al. Synergistic effects of the dopaminergic and glutamatergic system on hippocampal volume in alcohol-dependent patients. Biol Psychol. 2008;79(1):126-36.

76. Baker EJ, Walter NA, Salo A, Rivas Perea P, Moore S, Gonzales S, et al. Identifying Future Drinkers: Behavioral Analysis of Monkeys Initiating Drinking to Intoxication is Predictive of Future Drinking Classification. Alcohol Clin Exp Res. 2017;41(3):626-36.

77. Cosa A, Moreno A, Pacheco-Torres J, Ciccocioppo R, Hyytia P, Sommer WH, et al. Multimodal MRI classifiers identify excessive alcohol consumption and treatment effects in the brain. Addict Biol. 2016.

78. Bachmanov AA, Tordoff MG, Beauchamp GK. Ethanol consumption and taste preferences in C57BL/6ByJ and 129/J mice. Alcohol Clin Exp Res. 1996;20(2):201-6.

79. Ling J, Merideth F, Caprihan A, Pena A, Teshiba T, Mayer AR. Head injury or head motion? Assessment and quantification of motion artifacts in diffusion tensor imaging studies. Hum Brain Mapp. 2012;33(1):50-62. 
80. Power JD, Barnes KA, Snyder AZ, Schlaggar BL, Petersen SE. Spurious but systematic correlations in functional connectivity MRI networks arise from subject motion. Neuroimage. 2012;59(3):2142-54.

81. Lo K, Hahne F, Brinkman RR, Gottardo R. flowClust: a Bioconductor package for automated gating of flow cytometry data. BMC Bioinformatics. 2009;10:145.

82. Ashburner J. A fast diffeomorphic image registration algorithm. Neurolmage. 2007;38(1):95113.

83. Schwarz AJ, Danckaert A, Reese T, Gozzi A, Paxinos G, Watson C, et al. A stereotaxic MRI template set for the rat brain with tissue class distribution maps and co-registered anatomical atlas: Application to pharmacological MRI. Neurolmage. 2006;32(2):538-50.

84. Ashburner J, Friston KJ. Unified segmentation. Neurolmage. 2005;26(3):839-51.

85. Mourao-Miranda J, Bokde AL, Born C, Hampel H, Stetter M. Classifying brain states and determining the discriminating activation patterns: Support Vector Machine on functional MRI data. Neuroimage. 2005;28(4):980-95.

86. Nie B, Chen K, Zhao S, Liu J, Gu X, Yao Q, et al. A rat brain MRI template with digital stereotaxic atlas of fine anatomical delineations in paxinos space and its automated application in voxel-wise analysis. Human brain mapping. 2013;34(6):1306-18.

87. Rumple A, McMurray M, Johns J, Lauder J, Makam P, Radcliffe $M$, et al. 3-dimensional diffusion tensor imaging (DTI) atlas of the rat brain. PloS one. 2013;8(7):e67334.

88. Valdes-Hernandez PA, Sumiyoshi A, Nonaka H, Haga R, Aubert-Vasquez E, Ogawa T, et al. An in vivo MRI Template Set for Morphometry, Tissue Segmentation, and fMRI Localization in Rats. Frontiers in neuroinformatics. 2011;5:26.

89. Veraart J, Leergaard TB, Antonsen BT, Van Hecke W, Blockx I, Jeurissen B, et al. Populationaveraged diffusion tensor imaging atlas of the Sprague Dawley rat brain. Neurolmage. 2011;58(4):975-83.

90. Biedermann S, Fuss J, Zheng L, Sartorius A, Falfan-Melgoza C, Demirakca T, et al. In vivo voxel based morphometry: detection of increased hippocampal volume and decreased glutamate levels in exercising mice. Neuroimage. 61. United States: 2012 Elsevier Inc; 2012. p. 1206-12.

91. Smith SM. Fast robust automated brain extraction. Human Brain Mapping. 2002;17(3):14355.

92. Paxinos G, Watson C. The Rat Brain in Stereotaxic Coordinates: Elsevier Academic Press; 2005.

93. Smola AJ, Schölkopf B. On a kernel-based method for pattern recognition, regression, approximation, and operator inversion. Algorithmica. 1998;22(1):211-31. 
94. Vapnik VN. The Nature of Statistical Learning Theory. New York, NY, USA: Springer-Verlag New York, Inc; 1995.

95. Boser BE, Guyon IM, Vapnik VN. A training algorithm for optimal margin classifiers. Proceedings of the fifth annual workshop on Computational learning theory; Pittsburgh, Pennsylvania, USA. 130401: ACM; 1992. p. 144-52.

96. Schölkopf B, Smola A, Müller K-R. Nonlinear component analysis as a kernel eigenvalue problem. Neural computation. 1998;10(5):1299-319.

97. Mika S, Smola A, Schölkopf B, editors. An improved training algorithm for kernel fisher discriminants. Proceedings AISTATS; 2001: Morgan Kaufmann.

98. Platt J. Probabilistic outputs for support vector machines and comparisons to regularized likelihood methods. Advances in large margin classifiers. 1999;10(3):61-74.

99. Muller KR, Mika S, Ratsch G, Tsuda K, Scholkopf B. An introduction to kernel-based learning algorithms. IEEE Trans Neural Netw. 2001;12(2):181-201.

100. Lemm S, Blankertz B, Dickhaus T, Muller KR. Introduction to machine learning for brain imaging. Neuroimage. 2011;56(2):387-99.

101. Farahat AK, Ghodsi A, Kamel MS, editors. An efficient greedy method for unsupervised feature selection. Data Mining (ICDM), 2011 IEEE 11th International Conference on; 2007 2011: IEEE.

102. Kohavi R, John GH. Wrappers for feature subset selection. Relevance. 1997;97(1-2):273-324.

103. Jain AK, Duin RPW, Mao J. Statistical pattern recognition: A review. IEEE Transactions on pattern analysis and machine intelligence. 2000;22(1):4-37.

104. Li TQ, Wahlund LO. The search for neuroimaging biomarkers of Alzheimer's disease with advanced MRI techniques. Acta Radiol. 2011;52(2):211-22.

105. Feis D-L, Brodersen KH, von Cramon DY, Luders E, Tittgemeyer M. Decoding gender dimorphism of the human brain using multimodal anatomical and diffusion MRI data. Neuroimage. 2013;70:250-7.

106. Müller K-R, Krauledat M, Dornhege G, Curio G, Blankertz B. Machine learning techniques for brain-computer interfaces. Biomed Tech. 2004;49(1):11-22.

107. Breiman L. Classification and regression trees. 1984.

108. Breiman L. Random forests. Machine learning. 2001;45(1):5-32.

109. Strobl C, Boulesteix AL, Zeileis A, Hothorn T. Bias in random forest variable importance measures: illustrations, sources and a solution. BMC Bioinformatics. 2007;8:25. 
110. Smialowski P, Frishman D, Kramer S. Pitfalls of supervised feature selection. Bioinformatics. 2010;26(3):440-3.

111. Sonnenburg S, Rätsch G, Schäfer C, Schölkopf B. Large scale multiple kernel learning. Journal of Machine Learning Research. 2006;7(Jul):1531-65.

112. Schrouff J, Rosa MJ, Rondina JM, Marquand AF, Chu C, Ashburner J, et al. PRoNTo: pattern recognition for neuroimaging toolbox. Neuroinformatics. 2013;11(3):319-37.

113. Dempster AP, Laird NM, Rubin DB. Maximum likelihood from incomplete data via the EM algorithm. JOURNAL OF THE ROYAL STATISTICAL SOCIETY, SERIES B. 1977;39(1):1-38.

114. Harville DA. Maximum Likelihood Approaches to Variance Component Estimation and to Related Problems. Journal of the American Statistical Association. 1977;72(358):320-38.

115. Akaike H. Akaike's Information Criterion. In: Lovric M, editor. International Encyclopedia of Statistical Science. Berlin, Heidelberg: Springer Berlin Heidelberg; 2011. p. 25-.

116. Schwarz G. Estimating the dimension of a model. Annals of Statistics. 1978;6(2):461-4.

117. Smyth P. Model selection for probabilistic clustering using cross-validated likelihood. Statistics and Computing. 2000;10(1):63-72.

118. Stone M. Cross-validatory choice and assessment of statistical predictions. RoyStatSoc. 1974;36:111-47.

119. Box GEP, Cox DR. An analysis of transformations. Journal of the Royal Statistical Society, Series B. 1964;26(2):211-52.

120. Sfikas G, Nikou C, Galatsanos N, editors. Robust image segmentation with mixtures of Student's t-distributions. Image Processing, 2007 ICIP 2007 IEEE International Conference on; 2007: IEEE.

121. Nguyen TM, Wu QMJ. A fuzzy logic model based Markov random field for medical image segmentation. Evolving Systems. 2013;4(3):171-81.

122. Schwarz G. Estimating the dimension of a model. The annals of statistics. 1978;6(2):461-4.

123. Juntu J, Sijbers J, De Backer S, Rajan J, Van Dyck D. Machine learning study of several classifiers trained with texture analysis features to differentiate benign from malignant soft-tissue tumors in T1-MRI images. J Magn Reson Imaging. 2010;31(3):680-9.

124. Larroza A, Moratal D, Paredes-Sanchez A, Soria-Olivas E, Chust ML, Arribas LA, et al. Support vector machine classification of brain metastasis and radiation necrosis based on texture analysis in MRI. J Magn Reson Imaging. 2015;42(5):1362-8.

125. Jolliffe IT. Principal component analysis: Springer-Verlang; 1986. 
126. Genuer R, Poggi JM, Tuleau-Malot C. Variable selection using random forests. Pattern Recognition Letters. 2010;31(14):2225-36.

127. Ciccocioppo R, Economidou D, Cippitelli A, Cucculelli M, Ubaldi M, Soverchia L, et al. REVIEW: Genetically selected Marchigian Sardinian alcohol-preferring (msP) rats: an animal model to study the neurobiology of alcoholism. Addiction Biology. 2006;11(3-4):339-55.

128. Dietterich TG. Approximate statistical tests for comparing supervised classification learning algorithms. Neural computation. 1998;10(7):1895-923.

129. Uddin LQ, Menon V, Young CB, Ryali S, Chen T, Khouzam A, et al. Multivariate searchlight classification of structural magnetic resonance imaging in children and adolescents with autism. Biol Psychiatry. 2011;70(9):833-41.

130. Orru G, Pettersson-Yeo W, Marquand AF, Sartori G, Mechelli A. Using Support Vector Machine to identify imaging biomarkers of neurological and psychiatric disease: a critical review. Neurosci Biobehav Rev. 36. United States: 2012 Elsevier Ltd; 2012. p. 1140-52.

131. Cosa A, Canals S, Valles-Lluch A, Moratal D. Unsupervised segmentation of brain regions with similar microstructural properties: application to alcoholism. Conf Proc IEEE Eng Med Biol Soc. 2013;2013:1053-6.

132. Zhang D, Wang Y, Zhou L, Yuan H, Shen D, Alzheimer's Disease Neuroimaging I. Multimodal classification of Alzheimer's disease and mild cognitive impairment. Neuroimage. 2011;55(3):856-67.

133. Yang H, Liu J, Sui J, Pearlson G, Calhoun VD. A Hybrid Machine Learning Method for Fusing fMRI and Genetic Data: Combining both Improves Classification of Schizophrenia. Front Hum Neurosci. 2010;4:192.

134. Hinrichs C, Singh V, Xu G, Johnson SC, Alzheimers Disease Neuroimaging I. Predictive markers for $A D$ in a multi-modality framework: an analysis of $\mathrm{MCl}$ progression in the ADNI population. Neuroimage. 2011;55(2):574-89.

135. Fan Y, Resnick SM, Wu X, Davatzikos C. Structural and functional biomarkers of prodromal Alzheimer's disease: a high-dimensional pattern classification study. Neuroimage. 2008;41(2):27785.

136. Belknap JK, Crabbe JC, Young ER. Voluntary consumption of ethanol in 15 inbred mouse strains. Psychopharmacology (Berl). 1993;112(4):503-10.

137. Foran DR, Peterson AC. Myelin acquisition in the central nervous system of the mouse revealed by an MBP-Lac Z transgene. J Neurosci. 1992;12(12):4890-7.

138. Calabrese E, Johnson GA. Diffusion tensor magnetic resonance histology reveals microstructural changes in the developing rat brain. Neuroimage. 2013;79:329-39. 
139. Vetreno RP, Yaxley R, Paniagua B, Crews FT. Diffusion tensor imaging reveals adolescent binge ethanol-induced brain structural integrity alterations in adult rats that correlate with behavioral dysfunction. Addict Biol. 2016;21(4):939-53.

140. Zhang J, Jones MV, McMahon MT, Mori S, Calabresi PA. In vivo and ex vivo diffusion tensor imaging of cuprizone-induced demyelination in the mouse corpus callosum. Magn Reson Med. 2012;67(3):750-9.

141. Mori S, Zhang J. Principles of diffusion tensor imaging and its applications to basic neuroscience research. Neuron. 2006;51(5):527-39.

142. Felger JC, Miller AH. Cytokine effects on the basal ganglia and dopamine function: the subcortical source of inflammatory malaise. Front Neuroendocrinol. 2012;33(3):315-27.

143. Gilpin NW, Herman MA, Roberto $M$. The central amygdala as an integrative hub for anxiety and alcohol use disorders. Biol Psychiatry. 2015;77(10):859-69.

144. Koob GF. A role for brain stress systems in addiction. Neuron. 2008;59(1):11-34.

145. Ciccocioppo R, de Guglielmo G, Hansson AC, Ubaldi M, Kallupi M, Cruz MT, et al. Restraint stress alters nociceptin/orphanin $\mathrm{FQ}$ and CRF systems in the rat central amygdala: significance for anxiety-like behaviors. J Neurosci. 2014;34(2):363-72.

146. Poling J, Kosten TR, Sofuoglu M. Treatment outcome predictors for cocaine dependence. Am J Drug Alcohol Abuse. 2007;33(2):191-206.

147. Rosner S, Leucht S, Lehert P, Soyka M. Acamprosate supports abstinence, naltrexone prevents excessive drinking: evidence from a meta-analysis with unreported outcomes. J Psychopharmacol. 2008;22(1):11-23.

148. Foa EB, Yusko DA, McLean CP, Suvak MK, Bux DA, Jr., Oslin D, et al. Concurrent naltrexone and prolonged exposure therapy for patients with comorbid alcohol dependence and PTSD: $a$ randomized clinical trial. JAMA. 2013;310(5):488-95.

149. Anton RF, O'Malley SS, Ciraulo DA, Cisler RA, Couper D, Donovan DM, et al. Combined pharmacotherapies and behavioral interventions for alcohol dependence: the COMBINE study: a randomized controlled trial. JAMA. 2006;295(17):2003-17.

150. Garbutt JC, Kampov-Polevoy AB, Kalka-Juhl LS, Gallop RJ. Association of the Sweet-Liking Phenotype and Craving for Alcohol With the Response to Naltrexone Treatment in Alcohol Dependence: A Randomized Clinical Trial. JAMA Psychiatry. 2016;73(10):1056-63.

151. Komura Y, Tamura R, Uwano T, Nishijo H, Kaga K, Ono T. Retrospective and prospective coding for predicted reward in the sensory thalamus. Nature. 2001;412(6846):546-9.

152. Whelan R, Garavan H. When optimism hurts: inflated predictions in psychiatric neuroimaging. Biol Psychiatry. 2014;75(9):746-8. 
153. Deoni SCL. Quantitative relaxometry of the brain. Topics in Magnetic Resonance Imaging. 2010;21(2):101-13.

154. Khan AR, Goubran M, de Ribaupierre S, Hammond RR, Burneo JG, Parrent AG, et al. Quantitative relaxometry and diffusion MRI for lateralization in MTS and non-MTS temporal lobe epilepsy. Epilepsy research. 2013.

155. Budde MD, Xie M, Cross AH, Song SK. Axial diffusivity is the primary correlate of axonal injury in the experimental autoimmune encephalomyelitis spinal cord: a quantitative pixelwise analysis. J Neurosci. 29. United States2009. p. 2805-13.

156. Song SK, Yoshino J, Le TQ, Lin SJ, Sun SW, Cross AH, et al. Demyelination increases radial diffusivity in corpus callosum of mouse brain. Neuroimage. 26. United States2005. p. 132-40.

157. Mukherjee P, Berman JI, Chung SW, Hess CP, Henry RG. Diffusion tensor MR imaging and fiber tractography: theoretic underpinnings. AJNR Am J Neuroradiol. 2008;29(4):632-41.

158. Alexander AL, Lee JE, Lazar M, Field AS. Diffusion Tensor Imaging of the Brain. Neurotherapeutics. 2007;4(3):316-29.

159. Qin W, Zhang M, Piao Y, Guo D, Zhu Z, Tian X, et al. Wallerian Degeneration in Central Nervous System: Dynamic Associations between Diffusion Indices and Their Underlying Pathology. PLoS One. 2012;7(7).

160. Qin L, Crews FT. Chronic ethanol increases systemic TLR3 agonist-induced neuroinflammation and neurodegeneration. J Neuroinflammation. 2012;9:130.

161. He J, Crews FT. Increased MCP-1 and microglia in various regions of the human alcoholic brain. Exp Neurol. 2008;210(2):349-58.

162. Moslehi A, Nabavizadeh F, Dehpou A, Tavanga S, Hassanzadeh G, Zekri A, et al. Naltrexone attenuates endoplasmic reticulum stress induced hepatic injury in mice. Acta Physiologica Hungarica. 2014;101(3):341-52.

163. Smith JP, Bingaman SI, Ruggiero F, Mauger DT, Mukherjee A, McGovern CO, et al. Therapy with the opioid antagonist naltrexone promotes mucosal healing in active Crohn's disease: a randomized placebo-controlled trial. Digestive diseases and sciences. 2011;56(7):2088-97.

164. McLaughlin PJ, Immonen JA, Zagon IS. Topical naltrexone accelerates full-thickness wound closure in type 1 diabetic rats by stimulating angiogenesis. Experimental Biology and Medicine. 2013;238(7):733-43.

165. Biller A, Reuter M, Patenaude B, Homola GA, Breuer F, Bendszus M, et al. Responses of the Human Brain to Mild Dehydration and Rehydration Explored In Vivo by $1 \mathrm{H}-\mathrm{MR}$ Imaging and Spectroscopy. AJNR Am J Neuroradiol. 2015;36(12):2277-84. 
166. Dudek M, Canals S, Sommer WH, Hyytia P. Modulation of nucleus accumbens connectivity by alcohol drinking and naltrexone in alcohol-preferring rats: A manganese-enhanced magnetic resonance imaging study. Eur Neuropsychopharmacol. 2016;26(3):445-55. 
Multimodal MRI to identify pathological states in msP rats 


\section{Appendix I}

The following work was presented at the 35th Annual International Conference of the IEEE Engineering in Medicine and Biology Society (EMBC'13) celebrated in Kyoto (1).

COSA, A., CANALS, S., VALLES-LLUCH, A. \& MORATAL, D. 2013. Unsupervised segmentation of brain regions with similar microstructural properties: application to alcoholism. Conf Proc IEEE Eng Med

Biol Soc, 2013, 1053-6.

This work has two main objectives. Firstly, a novel segmentation method is proposed that complements traditional brain segmentation methods with a novel tissue classification based on similar microstructural properties derived from quantitative multimodal MRI. Secondly, the effects on brain microstructure associated to hard alcohol intake in a genetic rat model of alcoholism have been studied using the developed methodology.

\section{Methods}

\section{$\underline{\text { Animals }}$}

Drug naïve male rats of the Sardinian-marchigian alcohol preferring msP line (2) were obtained from the breeding facility at the University of Camerino, Italy. The breeding has continued for over 35 generations. Alcohol consumption in msP rats is $>5 \mathrm{~g} / \mathrm{kg} / \mathrm{day}$.

In total, 8 subjects were considered for this study. Animals were individually isolated each other for 30 days with 2 bottles: one full of water and the other full of EtOH diluted to $10 \%$ in water. Dissolution and water consumptions and weight were registered.

MR scans were acquired before and after alcohol consumption in order to evaluate changes between conditions.

\section{Data acquisition}

All images were acquired using a Bruker Biospec 7T (Bruker Biospin, Ettlingen, Germany). 
Anesthetized animals were placed in a modified saddle coil integrated within a customized stereotaxic animal holder. This allows precise positioning of the animal with respect to the coil and the magnet and avoids movement artifacts.

Fifteen axial slices were planned for every subject $\left(\mathrm{FOV}=32 \times 32 \mathrm{~mm}^{2}\right.$, matrix size $=128 \times 128$, in-plane resolution $=0.125 \times 0.125 \mathrm{~mm}^{2}$, slice thickness $=1 \mathrm{~mm}$ ).

Diffusion Tensor Imaging data was acquired using an Echo Planar Imaging diffusion sequence, with 30 uniform distributed gradient directions, $b=670 \mathrm{~s} / \mathrm{mm}^{2}$, with two non-diffusion weighted images, repetition time $(T R)=4000 \mathrm{~ms}$, echo time $(T E)=23 \mathrm{~ms}$.

T2 map images were acquired using a multi-slice multi-echo sequence $(T R=6000 \mathrm{~ms}, \mathrm{TE}=[12$ 24364860728496108120132144156168180192204216228240252264276288300312 324336348 360] ms).

T1 map images were acquired using a Rapid Acquisition with Relaxation Enhancement (RARE) sequence with variable repetition time (TE = $12.61 \mathrm{~ms}, \mathrm{TR}=\left[\begin{array}{l}155250400800160035006000\end{array} \mathrm{~ms}\right.$ ).

In order to facilitate spatial preprocessing steps, a three-dimensional (3D) image was acquired using a RARE sequence (field of view $=32 \times 32 \times 16 \mathrm{~mm}^{3}$, matrix size $=256 \times 128 \times 64$, voxel size of $\left.0.125 \times 0.25 \times 0.25 \mathrm{~mm}^{3}, \mathrm{TR}=1500 \mathrm{~ms}, \mathrm{TE}=9 \mathrm{~ms}\right)$.

\section{Preprocessing}

All same subjects' modalities were realigned using SPM 8 (Wellcome Trust Centre for Neuroimaging, University College London, London, UK). Rigid transformation parameters were calculated using non-diffusion volume (DTI), the largest repetition time volume (T2 map), and the shortest echo time volume (T1 map).

Three-dimensional images were re-scaled by a factor of 10 and skull stripped using BET (FMRIB Center, University of Oxford, Oxford, UK) (3). Before brain extraction, images were rescaled in the anterior-posterior direction by a factor of 0.5 , which made the rat brains more spherical. The output image was reversed to original size and shape. Calculated mask was used to delete non-brain tissue from other modalities. 
Spin-lattice relaxation time maps (T1 maps) and spin-spin relaxation time maps (T2 maps) were calculated using an in-house script written in MATLAB 7.1 (The MathWorks, Inc., Natick, MA, USA) fitting the data using a non-linear curve fit algorithm.

DTI were corrected for motion and eddy current distortion and fitted of local diffusion tensor using an available tool in FSL library for this purpose. From the diffusion tensor components, Fractional Anisotropy (FA) was determined.

In order to reduce confounding factors coming from the complex and heterogeneous anatomical structure of the brain couple with the inter-individual variability and the relatively low spatial resolution attainable (partial volume effects), a coronal slice located in a comparable brain position in each subject is only used in the study.

\section{Gaussian Mixture Model Definition}

There is a long tradition in the statistical literature of using finite mixture models (FMM) to perform probabilistic clustering allowing overlap of the clusters and handling uncertainty about cluster membership in a probabilistic way.

FMMs are weighted sums of a finite number of parametric probability density functions (pdfs) called component densities. A component models the probability of the data to belong to a certain class in an unsupervised classification problem. As MRI intensities distributions can be modeled as a Gaussian distribution, Finite Gaussian Mixture (FGM) models are widely used in MRI brain segmentation. FGM represents the probability of an intensity value $(\mathrm{x})$ given a set of parameters $(\Phi)$ and is defined as (1):

$$
\mathrm{p}(\mathrm{x} \mid \Phi)=\sum_{\mathrm{i}=1}^{\mathrm{k}} \alpha_{\mathrm{i}} \mathrm{g}\left(\mathrm{x} \mid \mu_{\mathrm{i}}, \Sigma_{\mathrm{i}}\right)
$$

where $\mathrm{k}$ is the number of the components in the model and $\alpha_{\mathrm{i}}$ are mixing parameters that models the prior probability of that class $\left(\sum_{\mathrm{i}=1}^{\mathrm{K}} \alpha_{\mathrm{i}}=1\right)$ while $\mathrm{g}\left(\mathrm{x} \mid \mu_{\mathrm{i}}, \Sigma_{\mathrm{i}}\right)$ is a Gaussian probability function defined by its mean $\mu_{\mathrm{i}}$ and covariance matrix $\Sigma_{\mathrm{i}}$ that represents probability of belonging to a specific class. 
The three microstructural parameters previously calculated have been used in the microstructural study of the brain. Let $\mathrm{x}=\{\mathrm{T} 1(\overrightarrow{\mathrm{r}}), \mathrm{T} 2(\overrightarrow{\mathrm{r}}), \mathrm{FA}(\overrightarrow{\mathrm{r}})\}$ be a 3-dimensional vector which represents the three microstructural values $(T 1, T 2$, and $F A)$ in a position of the brain $\vec{r} . X=$ $\left\{\mathrm{x}_{1}, \ldots, \mathrm{x}_{\mathrm{m}}\right\}$ is a random variable that represents $\mathrm{m}$ positions in the brain of a subject. $D$ denotes all the data observed in $\mathrm{N}$ subjects $\left(D=\mathrm{X}_{1} \cup \mathrm{X}_{2} \cup \ldots \cup \mathrm{X}_{N}\right)$. The set of parameters has been estimated using $D$.

Using Expectation Maximization (EM) algorithm (4) (5), maximum-likelihood parameters are obtained by the maximization mixture log-likelihood. The EM algorithm converges in parameter space to a local maximum of the objective function, but is no guarantee of convergence to global maximum. For avoiding convergence to local maximum, algorithm is repeated 100 times with different initial parameters. The set of parameters with the largest likelihood is assumed. Since the number of components in the model is unknown it has to be estimated. Penalized likelihood methods have often been used in model selection in mixture problem. In our case, we decided to use Bayesian Information Criterion (BIC) (6). BIC was evaluated in models from 1 to 30 components. Optimal value $\left(k^{*}\right)$ is chosen using the minimum value of $k$ to which BIC has suffered a $99 \%$ reduction of the difference between its maximum value and its asymptotic value. Using this criterion, it is assumed that model does not get complicated for improving convergence in $1 \%$. Also diagonal and unconstrained covariance matrices have been studied for modeling the data.

Finally, each voxel is classified in one of the components depending on the largest posterior probability of belonging to every component. In Figure 1, the proposed tissue classification framework is showed. 


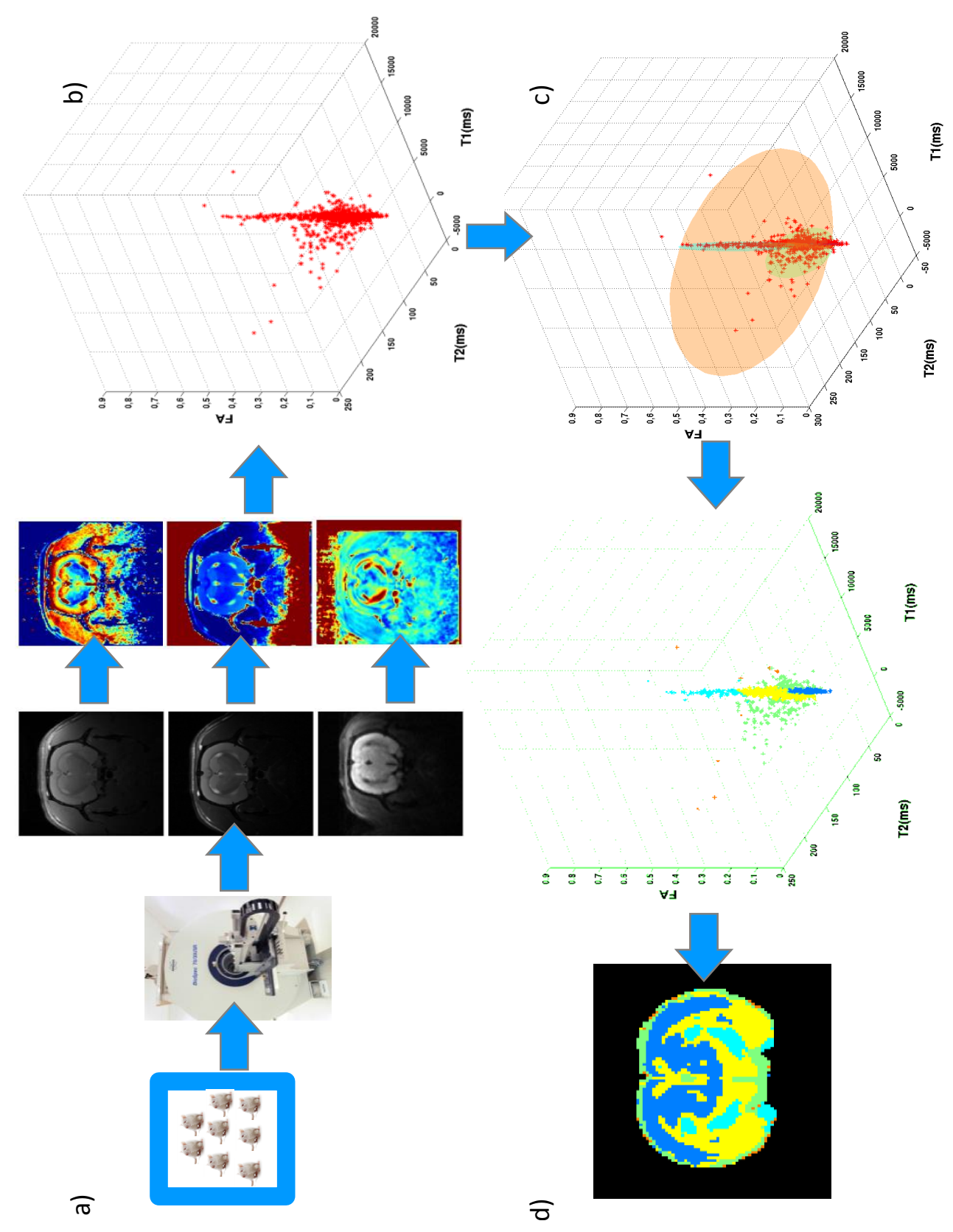

Figure 1. Framework. a) MR acquisition (T1 map, T2 map and DTI). b) Realignment and map calculation. Definition of subspace $D$. c) Model estimation with 5 components using EM algorithm. Each ellipsoid is related to every component of the model. d) Each observation (voxel) is labeled with its maximum posterior probability of belonging to each component.

\section{Subject Classifier}


Having defined the Finite Gaussian Model, a classifier based on linear discriminant analysis (LDA) is implemented and validated using a leave-one-out strategy. The classifier tries to distinguish between the two time points or conditions (before and after alcohol intake).

All subjects were characterized before and after alcohol intake by its mean microstructural parameter in each cluster. Both situations were compared by performing a t-test using as paired samples every microstructural mean value of each cluster before and after alcohol intake. Parameters which showed a statistically significant difference $(p<0.05)$ were used as an input for the classifier.

Goodness in the classification of the data is validated using a leave-one-out strategy. Each one of sixteen data sets (eight subjects and two conditions) is classified using the rest of them as training sample. Once all sets have been classified in one of the conditions, the hit rate or accuracy is defined as the ratio between the number of correctly classified sets and the total number of sets.

\section{$\underline{\text { Results and Discussion }}$}

In Figure 2, BIC values for diagonal and unconstrained covariance matrices are represented for different $k$ number of clusters. It is noteworthy to mention that BIC for unconstrained matrices is slightly lower than for the diagonal case. This is in concordance with the results obtained by Desco et al. (7) who suggest that unconstrained covariance matrices allow better modeling of voxels containing a partial volume effect. 


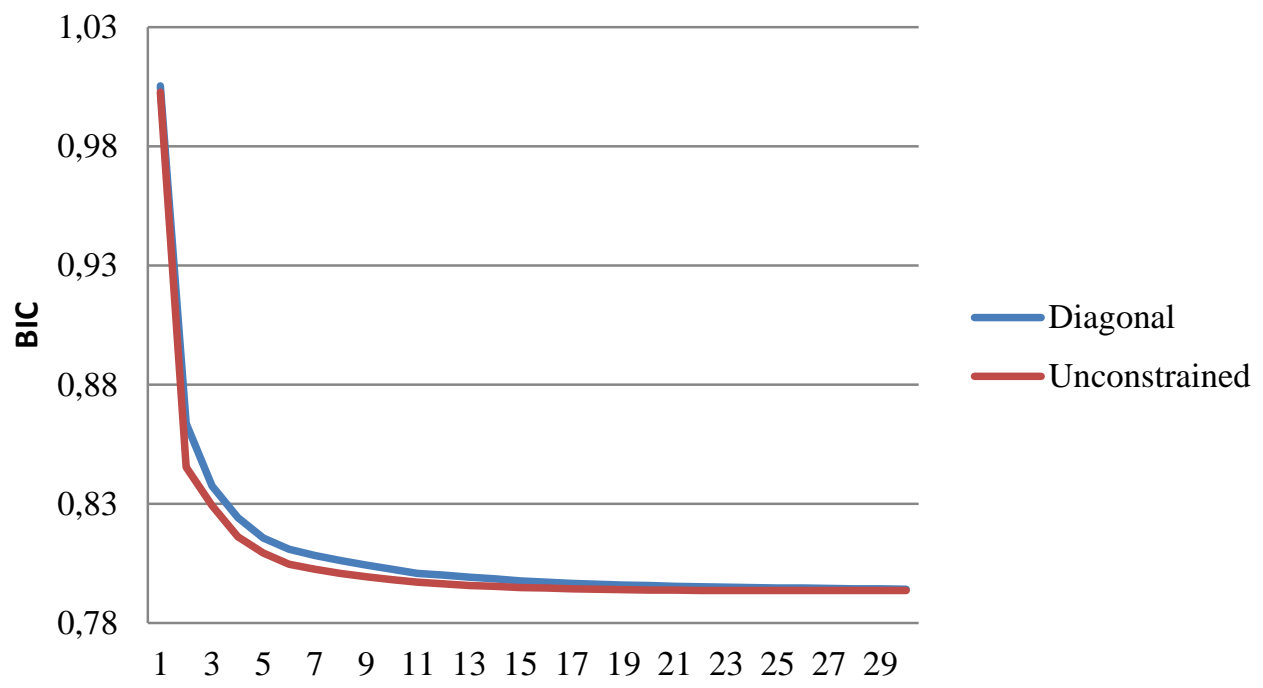

Figure 2. Bayesian Information Criterion (BIC) as a function of the number of clusters. In blue, using a unconstrained covariance matrix. In red, using a diagonal matrix.

Using the proposed criterion to define the optimal number of clusters, in $\mathrm{k}=14$ a reduction of $99 \%$ of the difference between the maximum and the asymptotic value is observed.

Once this point has been clarified, FGM model is defined using EM algorithm by 14 components and unconstrained covariance matrices.

Posterior probability of belonging to every component of each voxel is calculated. Each voxel is labeled depending on the largest posterior probability. In Figure 3, a map of the labels in a subject before and after alcohol intake is showed.

Each cluster can be characterized using the three mean microstructural values (T1, T2, and FA). Standard deviation of values in a voxel is highly related with the width of the Gaussian of the component.

Afterwards, a LDA classifier is implemented for clusters that present a statistically significant difference between the two time points (before/after alcohol intake) in their microstructural 
composition. Using a common $p$ threshold $(p<0.05)$, T1 maps present differences in clusters 2 $(p=0.047), 3(p=0.039), 4(p=0.024)$ and $5(p=0.024)$, while T2 maps show statistically significant differences in clusters $9(p=0.006)$ and $12(p=0.002)$. Fractional anisotropy did not present any difference using this presented model.

Using the model with 14 components, the hit rate is $93.75 \%$ of the cases, i.e. new subjects would be well-classified according to the evaluated microstructural parameters. High success rates are observed partially due to the inputs of the LDA algorithm, as they are microstructural values which have been chosen statistically different before and after hard alcohol consumption.

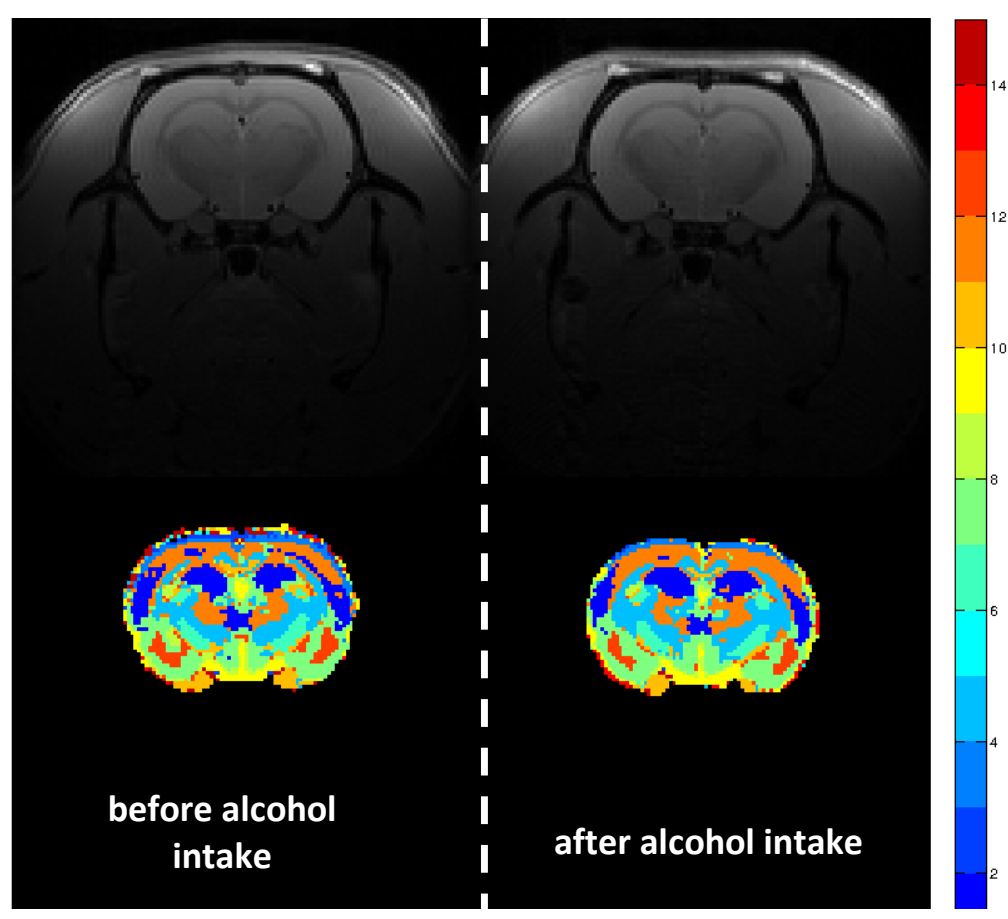

Figure 3. Map of labels of a subject before (left column) and after (right column) alcohol intake.

\section{Conclusions}

The hard-alcohol consumption effect in the brain can be studied using the microstructural information of tissues derived from MRI acquisition. 
In this work, a fuzzy tissue classification employing three microstructural parameters (T1 and T2 relaxation times and fractional anisotropy) widely used in the study of mental diseases is proposed. The distribution of quantitative parameters derived from MRI has been modeled by a finite weighted sum of multivariate Gaussian distribution where each component of the model represents a particular tissue in the brain.

The non-trivial problem of defining the number of clusters has been solved using the BIC value. Once the optimal number of components of the FGM has been calculated, the structure of covariance matrix of the model has been studied obtaining that the data are better classified if an unconstrained matrix is used. This conclusion is in accordance with other results that propose that partial volume effect due to the resolution of MR images can be modeled by non-diagonal matrix. After the model is defined, all voxels in the brain are classified using the largest posterior probability of belonging to each component obtaining a similar distribution of the clusters within the brain in all the cases.

Using microstructural information a Linear Discriminant classifier is implemented with the aim to identify subjects who have been exposed to alcohol and validated using a leave-one-out strategy. The $93.75 \%$ of subjects are correctly classified suggesting that the study of quantitative parameters derived from MRI are an important tool in the identification of areas related to hard alcoholconsumption effect in the brain.

\section{$\underline{\text { References }}$}

1. Cosa A, Canals S, Valles-Lluch A, Moratal D. Unsupervised segmentation of brain regions with similar microstructural properties: application to alcoholism. Conf Proc IEEE Eng Med Biol Soc. 2013;2013:1053-6.

2. Ciccocioppo R, Economidou D, Cippitelli A, Cucculelli M, Ubaldi M, Soverchia L, et al. Genetically selected Marchigian Sardinian alcohol-preferring (msP) rats: an animal model to study the neurobiology of alcoholism. Addict Biol. 2006;11(3-4):339-55.

3. Smith SM. Fast robust automated brain extraction. Hum Brain Mapp. 2002;17(3):143-55.

4. Dempster AP, Laird NM, Rubin DB. Maximum likelihood from incomplete data via the EM algorithm. JOURNAL OF THE ROYAL STATISTICAL SOCIETY, SERIES B. 1977;39(1):1-38. 
5. Jones P, McLachlan G. Algorithm AS 254: Maximum Likelihood Estimation from grouped and truncated data with finite normal mixture models. Journal of the Royal Statistical Society Series C (Applied Statistics). 1990;39(2):273-82.

6. Schwarz G. Estimating the dimension of a model. Annals of Statistics. 1978;6(2):461-4.

7. Desco M, Gispert JD, Reig S, Santos A, Pascau J, Malpica N, et al., editors. Statistical segmentation of multidimensional brain datasets. Medical Imaging 2001; 2001: International Society for Optics and Photonics. 